\title{
Controlling the Phase Behaviour and Photophysical Properties of Metallomesogens Through Structural Modification
}

\author{
by \\ William Albert Greenbank
}

\author{
A thesis \\ submitted to the Victoria University of Wellington \\ in partial fulfilment of the \\ requirements for the degree of \\ Master of Science with Honours \\ in Chemistry.
}

Victoria University of Wellington 



\section{Abstract}

Metallomesogens (metal-containing liquid crystals) have been of interest to chemists since the early 1980s. Since this period, many of the studies published on metallomesogens have focussed on the synthesis of novel metallomesogens, and studies of their phase behaviour. As a result there is a substantial body of knowledge of their synthesis and phase behaviour, however many of these studies have overlooked the interesting physical properties that transition metals or lanthanides may impart to the mesophase (liquid crystal state). The studies that have been carried out suggest that the optical and photophysical properties resulting from their self assembly are very different to those observed in the crystalline or isotropic liquid phases, and are highly dependent on the structure of the mesophase.

A series of salicylaldimine-based ligands and copper(II) complexes with a variety of structural modifications were synthesised and characterised. The structure, phase behaviour and phase relaxation kinetics of these compounds in the crystalline state were studied using differential scanning calorimetry (DSC), single crystal X-ray crystallography and variable temperature powder X-ray diffraction. The mesomorphism of the compounds was studied using small angle X-ray scattering (SAXS) and polarised optical microscopy (POM). The photophysical properties of the complexes and ligands were studied in the solution phase using ultraviolet-visible (UVvis) spectroscopy.

It was found that the smallest complexes (copper(II) N-alkyl,4-alkoxysalicylaldimine complexes) were not metallomesogens, but did exhibit multiple crystalline phases that formed as a result of changes in the conformation of the $\mathrm{N}$-alkyl chains. The transition temperatures of these crystalline phase changes were strongly dependent on the length of the alkyl chains due to kinetic phenomena. The extension of the rigid core of the complex via synthesis of an $\mathrm{N}$-(4-butylphenyl) derivative was successful in inducing mesomorphism in both the complex and the ligand. The ligand formed an enantiotropic nematic mesophase, while the complex formed a monotropic smectic $A$ mesophase. The structural differences between the non-mesomorphic complexes, the mesomorphic ligand and the mesomorphic complex indicate that the determining 
factor in the formation of mesophases is the magnitude of lateral interactions between the molecules, which is governed by the size and shape of the rigid core. Further attempts at inducing mesomorphism by formation of bimetallic copper complexes were unsuccessful due to chemical instability.

The photophysical properties of the compounds showed that the salicylaldimine ligands exist in solution in a tautomeric equilibrium, which can be influenced by the hydrogen-bonding character of the solvent. The ligands also show evidence of photochromism, while the complexes exhibit LMCT bands, both features which could affect and be affected by self assembly. It was also determined from their UV-vis spectra and DFT studies that the ligands bind to the metal centre in a manner which is intermediate to the two tautomeric forms, but close to the higher energy keto-amine tautomer.

These results demonstrate that structural modification can be used to control both the phase behaviour and physical properties of salicylaldimine complexes. The compounds studied here also show potential to exhibit a variety of self assembly-dependent photophysical properties in the mesophase and would be good candidates for future research in this area. 


\section{Acknowledgements}

One of the more frustrating aspects of writing this thesis has been the constant need to refer to things in the impersonal tense, as if the results presented here materialised out of mid air! Because I've been writing this way for so long, I will have to make a conscious effort to avoid reverting to it and saying, "... and the author wishes to thank..." in order to live up to the expectations of a certain past member of the Soft Matter and Biomineralization research group regarding "bland and emotionless" acknowledgements sections. ${ }^{1}$

Of course, it isn't true that this work materialised out of thin air, it came about through the help and input of a great number of people, only a few of whom I have the space to thank here. First and foremost, thanks must go to my supervisor, Professor Kate McGrath. Without her wisdom, guidance and patience (of which she has needed plenty) over the last two years I would not have even come close to getting to this point. I also want to thank everyone currently and previously in the Soft Matter and Biomineralization research group with me. Natasha, Graham, Riyad, Davoud, Mehrdad, Marjorie, Saeedeh, Nurul, Mario, Alex, Ivan (especially for your help with the computational chemistry), Agathe, Nick and Ash, you have all been fantastic to work with and immensely helpful when I've needed it.

I'd also like to thank a number of other people who have helped me over the last two years. Dr Shane Telfer for all his help with the X-ray experiments and Massey University for the use of their equipment. Dr Joanne Harvey and Jackie King for the use of their labs and equipment during the synthesis part of my project, as well as the Organic Synthesis group for being patient with me when I needed to use their rotavaps and everyone else at SCPS I borrowed chemicals and equipment off. A big thank you also goes to all the technical staff at SCPS for keeping all the instruments and equipment I needed running and fixing the things I broke. I also need to thank the Victoria Graduate Award, the J. L. Stewart Scholarship and the Curtis-Gordon Research Scholarship in Chemistry for funding. 
Finally, but most importantly, I must thank my parents Christine and lan, my brother Richard, my sister Emma and my partner Fern. Without all of your love, support and advice I could not have done any of this. 


\section{Table of Contents}

Abstract

i

Acknowledgements

iii

Iable of Contents $\mathbf{v}$

Naming Conventions of Compounds vii

List of Abbreviations viii

Chapter 1 Introduction 1

1.1. Overview

1.2. Research Proposal

2.1. Introduction

2.2. Thermotropic Liquid Crystals

3.1. Introduction

3.2. The Role of the Metal

3.3. Ligand Design

3.4. Electrical and Optical Properties

3.5. Luminescent and Electronic Properties

4.1. Introduction

4.2. 4-Alkoxysalicylaldimine Ligands

4.3. Mononuclear Complexes

4.4. Binuclear Complexes

4.5. Experimental 
5.1. Introduction 39

5.2. Analysis Techniques and Instrumentation $\quad 40$

5.3. Mononuclear N-alkyl Salicylaldimine Complexes Cul 42

5.4. Binuclear N-alkyl Salicylaldimine Complexes Cu2 55

5.5. N-(4-butylphenyl) Salicylaldimine Complex and Ligand 57

5.6. Conclusions 71

Chapter 6 Solution Phase Photophysical Behaviour $\quad 73$

6.1. Introduction 73

6.2. Spectral Features of Ligands $\quad 74$

6.3. Spectral Features of Complexes 82

6.4. Conclusions and Future Prospects 89

Chapter 7 Conclusions and Future Work $\quad 93$

7.1. Conclusions 93

7.2. Future Work 95

Glossary $\quad 97$

Appendix A Introduction to DSC and POM 99

A.1. Differential Scanning Calorimetry (DSC) 99

$\begin{array}{ll}\text { A.2. Polarised Optical Microscopy (POM) } & 101\end{array}$

\begin{tabular}{lr} 
References & 105 \\
\hline
\end{tabular} 


\section{Naming Conventions of Compounds}

For the most part, IUPAC nomenclature will be adhered to in this thesis; however abbreviated notation will be used to refer to the ligands and copper(II) complexes synthesised in this project. This notation is summarised in Figure 0.1.
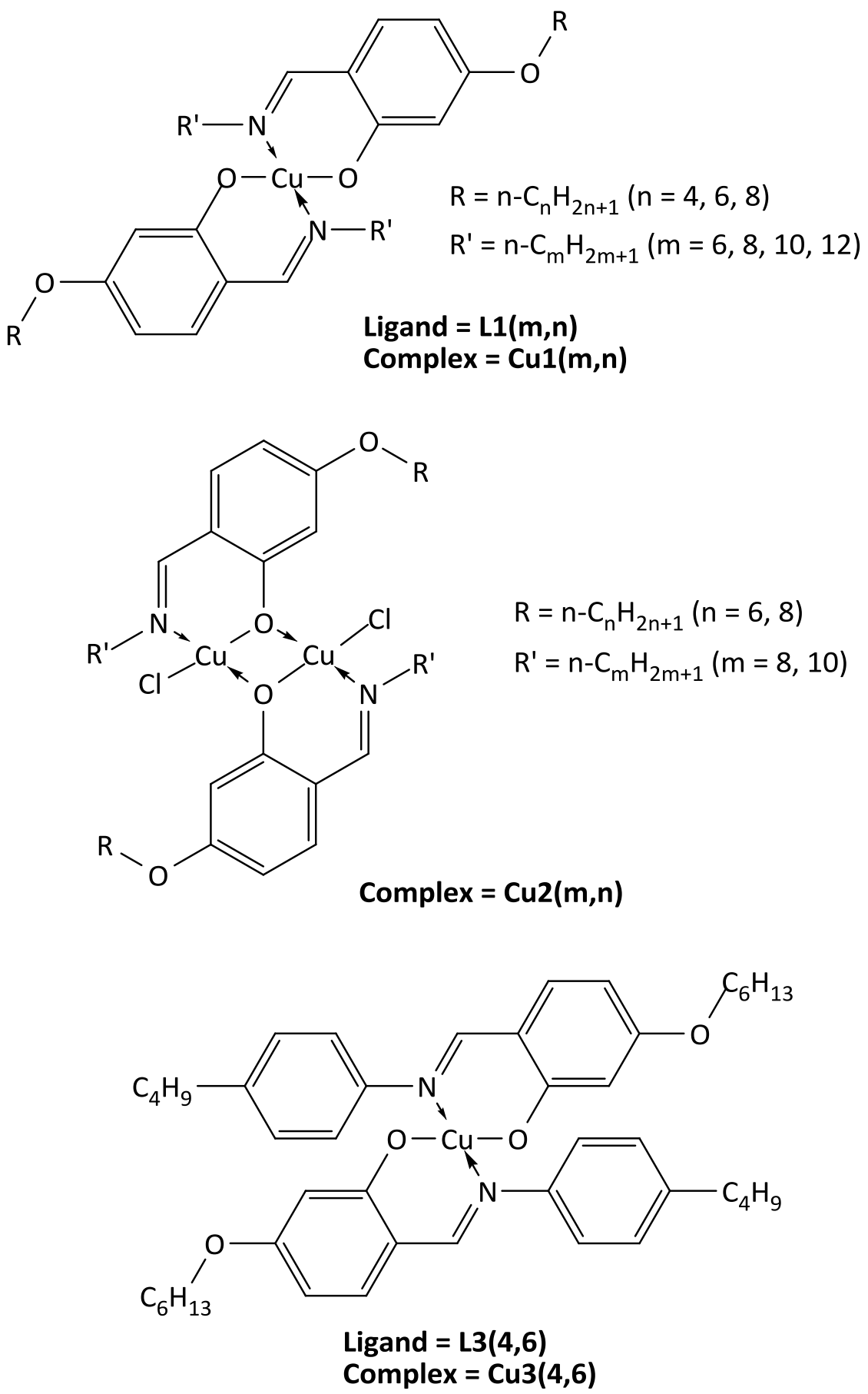

Figure 0.1: The naming conventions for the copper(II) metal complexes and ligands synthesised in this project. 


\title{
List of Abbreviations
}

\author{
AI(P)E Aggregation induced (phosphorescence) emission \\ AR Analytical reagent \\ COSY Correlation spectroscopy \\ (TD-)DFT (Time dependent) density functional theory \\ DSC Differential scanning calorimetry \\ FTIR Fourier transform infrared (spectroscopy) \\ FWHM Full-width half maximum \\ HMBC Heteronuclear multiple bond coherence \\ HSQC Heteronuclear single quantum correlation \\ IR Infrared \\ LC Liquid crystal \\ LCD Liquid crystal display \\ LMCT Ligand to metal charge transfer \\ MBBA N-(4-methoxybenzylidene)-4-butylaniline \\ MLCT Metal to ligand charge transfer \\ MLLCT Metal to ligand to ligand charge transfer \\ NMR Nuclear Magnetic Resonance \\ POM Polarised optical microscopy \\ PXRD Powder X-ray diffraction \\ RF Retention Factor \\ salen $N, N^{\prime}$-disalicylideneethylenediamine \\ SAXS Small angle X-ray scattering \\ TLC Thin layer chromatography \\ UV-vis Ultraviolet-visible \\ WSAXS Small/wide angle X-ray scattering \\ $X R D \quad X$-ray diffraction
}




\section{Chapter 1 Introduction}

\subsection{Overview}

Metal-containing mesogens, or metallomesogens, have been of interest to chemists since the early 1980s. This was as a result of the significant growth of interest in liquid crystals that occurred in the mid to late 1970s due to the discovery of mesogens that were mesomorphic at room temperature in 1969. ${ }^{2,3}$ Much of the interest in metallomesogens was centred on the possibility of their incorporation into liquid crystal displays, although it quickly became apparent that this was not possible with conventional LCDs (vide infra). Since this period, many of the studies published on metallomesogens have focussed on the synthesis of novel metallomesogens and their phase behaviour. Although there are still many questions to be answered regarding the phase behaviour of metallomesogens, these studies have built up a substantial body of knowledge of the synthesis and phase behaviour of metallomesogens.

These synthetic studies do, however, often overlook the physical properties that the incorporation of a transition metal or lanthanide may impart to the mesophase (liquid crystal phase). Their magnetic, electronic, luminescent, optical and electrical properties are often unique to the mesophase, and are not observed in either the crystalline or isotropic liquid phases. As such their study allows an insight into new aspects of self assembly. Of particular interest to this study is the use of self assembly to control the physical properties of the mesophases of metallomesogens. This contrasts with the "traditional" approach that chemists have taken to control the physical properties of a system by variations in the chemical structure alone. The advantage of using self assembly is the possibility that the same system can produce different physical properties through the use of different external stimuli which alter the self assembly.

This does not, of course, mean that chemical structure is unimportant, as ultimately it is the chemical structure that directs the self assembly of the mesogens. It is through the combination of knowledge of the effects of mesogen structure and an 
understanding of the effects of self assembly on the physical properties of the mesophase that one may begin to use self assembly to control the physical properties of a system. While there have been numerous studies of the former, the latter has not been explored to the same extent. In this study, the relationship between chemical structure and phase behaviour was explored for a range of mesomorphic and nonmesomorphic salicylaldimine-copper(II) complexes and their ligands, with particular emphasis on the use of chemical structure to direct self assembly. In addition, the solution phase electronic absorption properties of the complexes and ligands were examined to correlate their photophysical properties to their chemical structure and in turn suggest how they might be affected by self assembly. Although the phase behaviour and structure, and photophysical properties are examined separately in the text, they should not be considered in isolation as realisation of the objectives of this research requires a holistic understanding of the interrelationship between physical properties, phase behaviour and chemical structure.

\subsection{Research Proposal}

The aim of this research was to investigate the relationships between the structures of mesophases of metallomesogens, their physical properties, and their chemical structure through structural modification. In particular, this study aimed to do the following:

- synthesise of a series of metallomesogens that are mesomorphic over a wide range of temperatures, using literature methods;

- investigate the phase behaviour of the synthesised compounds as a function of structural modifications;

- and investigate the physical properties of the synthesised compounds, their relationship to the structure of the compound and the factors that influence this relationship. 


\section{Chapter 2 \\ Liquid Crystals}

\subsection{Introduction}

The term "liquid crystal" refers to substances that exhibit intermediate phases, called mesophases, between the crystalline and liquid phases. These mesophases are distinct from the crystalline and isotropic liquid phases in that they exhibit partial long-range order in one or more dimensions, but are also fluid. ${ }^{4}$ As such they can be considered to be anisotropic liquids, and often this is emphasised by the use of the term isotropic liquid to describe the liquid phase. ${ }^{5}$

The discovery of liquid crystals is widely attributed to Friedrich Reinitzer ${ }^{4,6}$ (although the phenomenon was observed before then in stearin by Wilhelm Heintz in $1850^{7}$ ) when in 1888 he observed that cholesterol benzoate appeared to have two melting points, one to a cloudy liquid (later recognised as a cholesteric phase - see Section 2.2.1) and one to a clear liquid. Although this generated much interest at the time, the compounds synthesised all had mesophases at high temperatures and as a result research into liquid crystals did not persist due to (at the time) a lack of practical applications. $^{8}$ However, the field was revitalised in 1969 when Hans Kelker synthesised $\mathrm{N}$-(4-Methoxybenzylidene)-4-butylaniline (MBBA), which was the first compound to exhibit mesomorphic behaviour at room temperature $\left(20^{\circ} \mathrm{C}\right.$ to $\left.41^{\circ} \mathrm{C}\right) .^{2}$ This led to a dramatic increase of interest in the field, which was further stimulated by high value applications in display technology, such as the liquid crystal display or LCD (now a 100 billion dollar industry ${ }^{9}$ ).

There are two main types of liquid crystals, classified by the manner in which the longrange order in the crystalline phase is destroyed. ${ }^{5}$ In thermotropic liquid crystals, the order is destroyed by heat alone, meaning that the mesophase behaviour is controlled by temperature. In lyotropic liquid crystals it is both the action of a solvent and temperature that results in the formation of mesophases. Since this research focuses on thermotropic metallomesogens (liquid crystalline materials which incorporate metals), lyotropic liquid crystals will not be discussed any further, however for a 
thorough review of lyotropic metallomesogens, the reader is referred to Donnio's review in Current Opinion in Colloid and Interface Science. ${ }^{10}$

\subsection{Thermotropic Liquid Crystals}

Compounds that behave as thermotropic liquid crystals form mesophases that mimic the structural anisotropy present in their constituent molecules. ${ }^{4}$ As such, in order for a compound to form mesophases, the compound must have a degree of structural anisotropy. This is often manifested as a rigid core moiety of the molecule. In addition to a rigid core, the molecule must also possess sufficient conformational degrees of freedom to prevent direct crystallisation from the liquid phase. This can be achieved by attaching multiple flexible tails, which are typically long alkyl chains, to the core moiety. In thermodynamic terms, the conformational entropy of the tails competes with the enthalpy of crystallisation. The interplay between the size of the highly anisotropic core and the length of the tails (which increases the degrees of freedom and thus the conformational entropy) is extremely delicate and seemingly small structural changes can have large effects on the mesophases exhibited by compounds. ${ }^{11}$ This will be discussed in more depth when metallomesogens are examined in Chapter 3.

A wide range of materials exhibit thermotropic mesophases. Typically these materials are classified by the shapes of the cores of their molecules (referred to as mesogens). For example, a mesogen with a rod-like core is classified as a calamitic mesogen and a mesogen with a disc-like core is referred to as a discotic mesogen. Since these are the two most prevalent types of thermotropic liquid crystal, ${ }^{4}$ they will be examined in more detail.

\subsubsection{Calamitic Mesogens}

Calamitic mesogens can exhibit a number of different mesophases with varying degrees of orientational and translational order. Whilst translational order can be easily defined through the use of lattice vectors, orientational order must be defined by a new parameter, known as the director. The director $(\mathbf{n})$ is a unit vector that describes the orientation of the mesogens, and is defined differently depending on the type of mesogen. In the case of calamitic mesogens, the director is generally defined as being parallel to the long axis of the rod, as shown in Figure 2.2.1. 

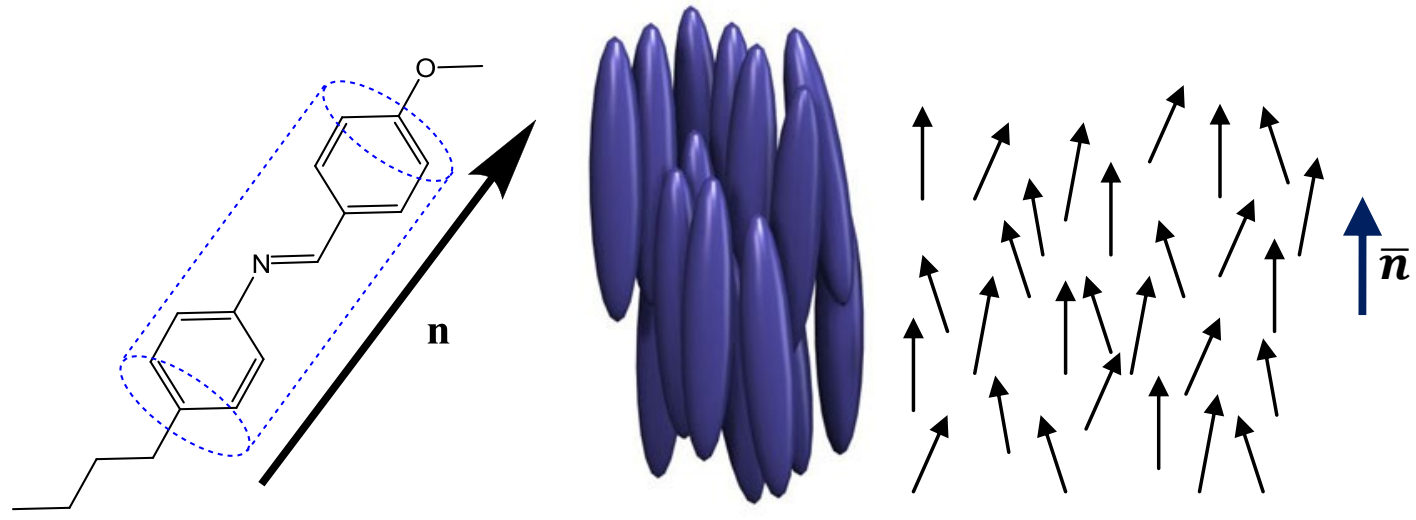

Figure 2.2.1: (left) An example of a calamitic mesogen (MBBA) with its rod shape and the director illustrated; (centre) an illustration of the nematic phase (reproduced from Yager - "Introduction to Liquid Crystals"12); and (right) the orientation of the directors in the nematic phase with the average director shown in blue.

Calamitic mesogens can form several different mesophases. Among these is the nematic mesophase, which is shown in Figure 2.2.1. The nematic mesophase is the most disordered of the thermotropic mesophases, the mesogens in the nematic mesophase have no translational order, but still have orientational order. This should not, however, be taken to mean that the mesogens are perfectly aligned. As this is a fluid, mesogens are constantly moving and rotating, however when averaged across the sample, the directors' average alignment is about a single axis. ${ }^{13}$

Calamitic mesogens can also form more ordered phases called smectic mesophases. These mesophases, like nematic mesophases, possess orientational order, but in addition they also possess translational order along one dimension. Physically, this means that the smectic phase is a "layered liquid" where mesogens are arranged in layers, but do not have long-range translational order within the layers. ${ }^{13}$ As with the nematic mesophase, the mesogens' directors average to align about a single axis. In smectic A mesophases the alignment axis is aligned with the normal axis of the planes, and in smectic $C$ mesophases the alignment axis is tilted with respect to the normal axis by a characteristic tilt angle $\boldsymbol{\omega}$. Both of these mesophases are shown in Figure 2.2.2.

In the cases where the mesogen is a chiral molecule, or has a chiral substance dissolved in it, ${ }^{4}$ chiral mesophases are induced. In the case of a nematic mesophase, a chiral nematic mesophase is more generally known as a cholesteric mesophase. Unlike a normal nematic phase, in which the average director is the same throughout the sample, the average director twists around a perpendicular axis in a helical fashion. ${ }^{4}$ As one might expect, the direction of the twist is opposite for opposite enantiomers. The 
degree to which the average director twists is expressed by the pitch of the mesophase $(p)$, which is the repeat distance of the helix. Sometimes the spatial period (L), which is half of the pitch, is quoted instead of the pitch because in a nematic phase $\overline{\boldsymbol{n}}$ and $-\overline{\boldsymbol{n}}$ are indistinguishable. ${ }^{13}$
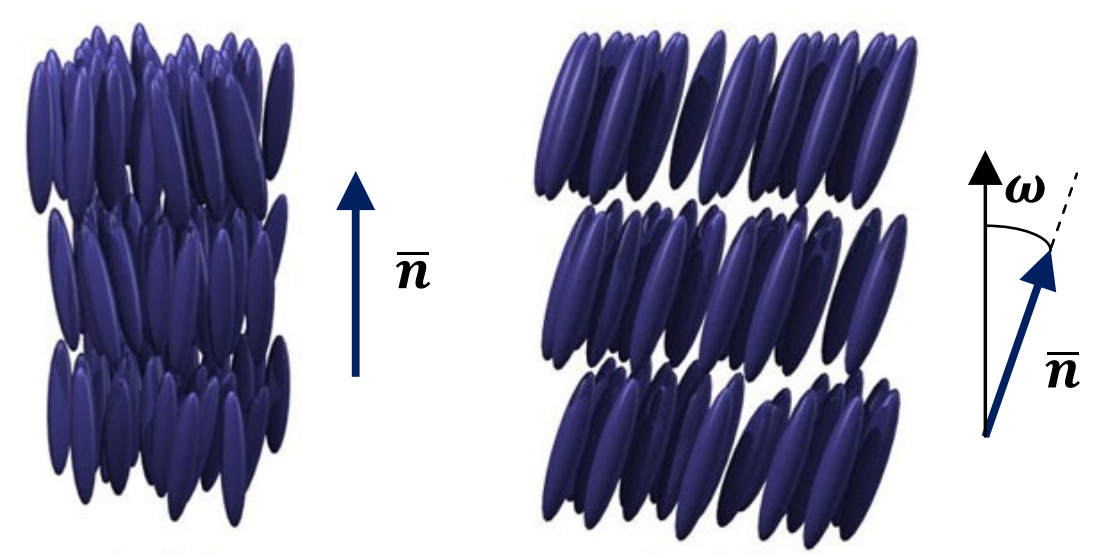

Figure 2.2.2: (left) A smectic A mesophase with the average director illustrated; and (right) a smectic C phase with the average director and tilt angle illustrated (adapted from Yager - "Introduction to Liquid Crystals"12).

The variation of the average director through the phase can be represented by:

$$
\overline{\boldsymbol{n}}=\sin \left(q_{0} z+\phi\right) \hat{\boldsymbol{\imath}}+\cos \left(q_{0} z+\phi\right) \hat{\boldsymbol{\jmath}} \quad \text { where } q_{0}=\frac{2 \pi}{p}
$$

where $z$ is defined as the axis of the helix, $\hat{\imath}$ and $\hat{\boldsymbol{j}}$ are unit vectors aligned with the $\mathrm{x}$ and $y$ axes and $\phi$ is an arbitrary phase factor. The spatial period of a cholesteric mesophase is typically much larger than molecular length scales ( $300 \mathrm{~nm})$. This means that it is of a similar magnitude to the wavelength of visible light, which can result in Bragg scattering. ${ }^{13}$ Reinizer mentioned this phenomenon in his 1888 paper in Monatshefte für Chemie when he wrote "it struck me that the substance in this case melted not into a clear transparent liquid but rather always into a cloudy liquid which was only translucent... it was also noted that when the substance was heated to a higher temperature, the clouding suddenly vanishes" ${ }^{6,8}$ An illustration of a cholesteric phase is shown in Figure 2.2.3.

Chiral mesogens can also form chiral smectic mesophases. The phase that is typically observed is the smectic $C^{*}$ phase. In the smectic $C^{*}$ mesophase, the average director is still tilted with respect to the normal axis of the plane, but also spatially precesses around the normal axis in a helical fashion ${ }^{13}$ (similar to the helical symmetry in a cholesteric mesophase). This can be described by a similar equation to the cholesteric mesophase: 


$$
\overline{\boldsymbol{n}}=\sin (\omega) \sin \left(q_{0} z+\phi\right) \hat{\boldsymbol{\imath}}+\sin (\omega) \cos \left(q_{0} z+\phi\right) \hat{\boldsymbol{\jmath}}+\cos (\omega) \widehat{\boldsymbol{k}} \quad \text { where } q_{0}=\frac{2 \pi}{p}
$$

where the $z$ axis (see Figure 2.2.3) is defined along the normal axis to the smectic plane, $\widehat{\boldsymbol{k}}$ is a unit vector along the $z$ axis, $\omega$ is the tilt angle (in radians) and all other terminology is the same as in the equation for the cholesteric mesophase. An illustration of the smectic $C^{*}$ phase is shown in Figure 2.2.4.
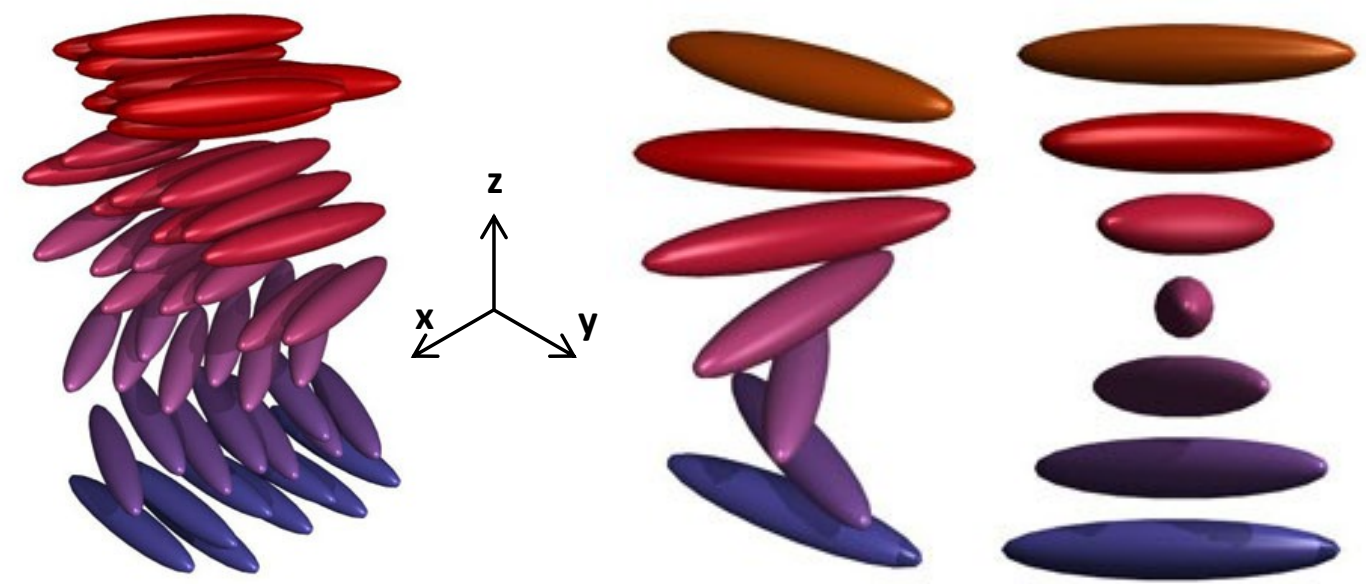

Figure 2.2.3: (left) An illustration of the cholesteric mesophase with the $x, y$ and $z$ axes defined, and (right) a simplified illustration (reproduced from Yager - "Introduction to Liquid Crystals"12). Note that in both diagrams, colour is only used to indicate the altitude of the mesogen in the diagram.

There are a number of other, rarer, mesophases formed by calamitic mesogens. ${ }^{4}$ These include the so-called blue mesophases. Blue mesophases form as an intermediate between a cholesteric mesophase and the isotropic liquid phase in some highly chiral mesogens. ${ }^{13}$ Their phase structure is believed to consist of a fluid cubic structure in the director field (a vector field defined by the orientation of the mesogen directors). ${ }^{14}$ There is some interest in their application as tuneable photonic crystals as their structure can be easily manipulated using electric fields. ${ }^{15}$ They were also referred to by Reinitzer in 1888 who recorded that a "deep violet-blue colour appears at one point. This spreads rapidly over the entire substance and vanishes again almost equally rapidly". ${ }^{6,8}$ As noted by Reinitzer, the blue mesophases typically form over a very narrow temperature, which limits their applications. There have been some recent advances in this regard, with some examples of blue mesophases extending from $60^{\circ} \mathrm{C}$ to $16^{\circ} \mathrm{C}^{14}$ and in 2008 Samsung developed the first LCD display to utilise blue phases. ${ }^{16}$ 


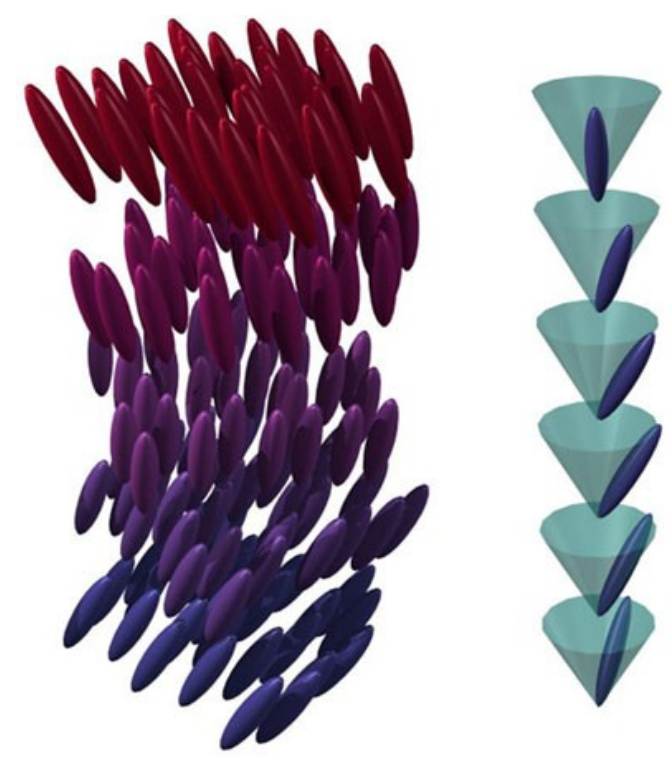

Figure 2.2.4: An illustration of the spatial director precession in a smectic $C^{*}$ mesophase (reproduced from Yager "Introduction to Liquid Crystals"12).

\subsubsection{Discotic Mesogens}

As with calamitic mesogens, discotic mesogens form a variety of mesophases. The orientational order in these mesophases is also defined using a director vector, however in discotic mesogens, this is defined as being a normal vector to the plane of the disc $^{13}$ (see Figure 2.2.5). Another similarity they share with calamitic mesogens is the formation of a nematic mesophase, which has the same characteristics as the calamitic variant and is shown in Figure 2.2.5.
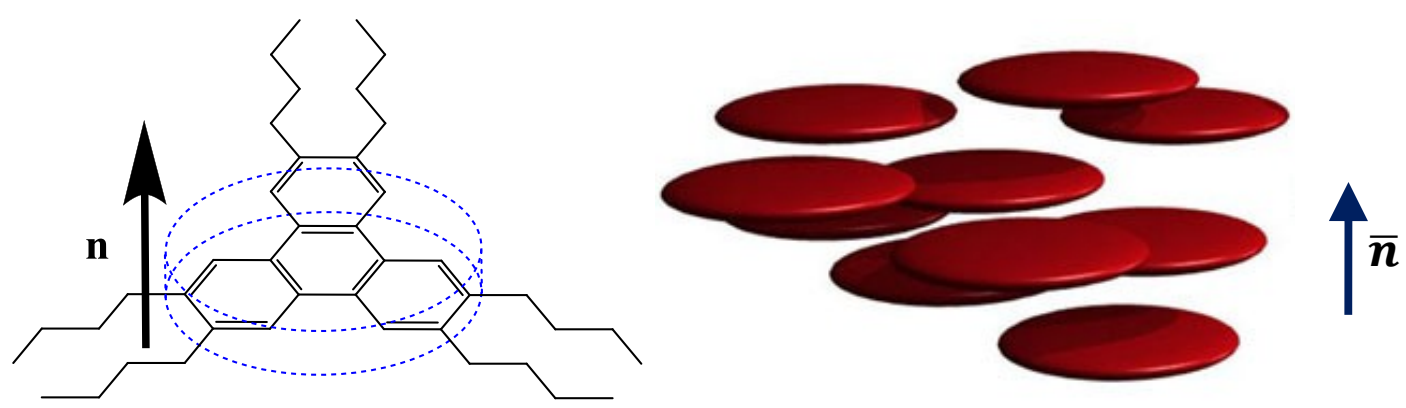

Figure 2.2.5: (left) An example of a discotic mesogen with the "disc shape" and the director illustrated, and (right) an illustration of a discotic nematic phase (reproduced from Yager - "Introduction to Liquid Crystals"12) with the average director shown.

Another series of mesophases that discotic mesogens can form are the columnar mesophases. Columnar mesophases are more ordered than smectic mesophases in that they have two dimensions of order (instead of one) and orientational order. As one might infer from the name, mesogens in columnar mesophases assemble into columns, within which there is no long-range order along the column's axis. ${ }^{13}$ The columnar mesophases are classified by the geometry of the packing of the columns, for example the mesophase in which the columns do not display any translational 
order is known as a nematic columnar mesophase. ${ }^{5}$ More ordered columnar mesophases include the hexagonal, rectangular and oblique mesophases, examples of which are shown in Figure 2.2.6. It should be noted that in rectangular and oblique mesophases, rather than being elliptical in shape, the mesogens are tilted with respect to the column axis. ${ }^{17}$

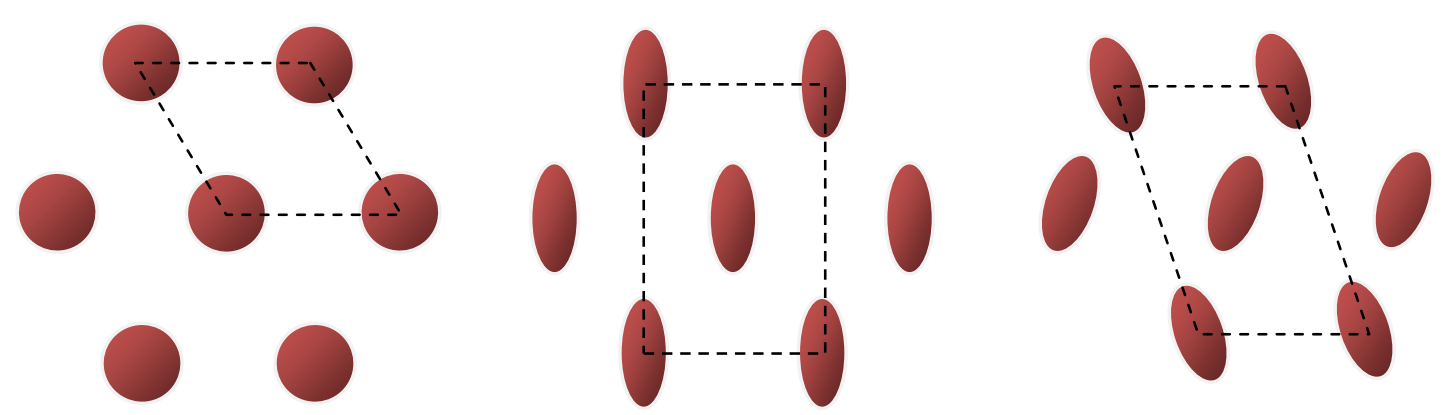

Figure 2.2.6: Illustrations of the hexagonal (left), rectangular (centre) and oblique (right) columnar mesophases, as viewed down the column axis. The dotted lines indicate the $2 D$ unit cell of the column packing. 


\section{Chapter 3}

\section{Metallomesogens and their Properties}

\subsection{Introduction}

Having discussed the generalities of liquid crystals in Chapter 2, we must now turn our attention to how the structural requisites of mesophase formation may be incorporated into metal-organic compounds, and what physical properties those mesophases have. Metallomesogen design has the same goals as the design of organic liquid crystals, i.e. a rigid, anisotropic core with long flexible tails. The incorporation of a metal into the compound can present design challenges, but also can allow for new design possibilities that are not available with organic compounds. ${ }^{3}$

These new characteristics can give rise to unique physical properties in the mesophases. These are often unique to the mesophase and arise from combining the partial order of a mesophase, with the properties of an metal-organic compound. In some cases they represent rare examples of fluids which exhibit physical characteristics typically only observed in crystalline solids. ${ }^{18,19}$ The properties that are of particular interest to researchers include electrical, optical, electronic, luminescent, and magnetic properties. As the reader will observe in Chapter 5 and Chapter 6 , this study does not examine all of these properties, so only those relevant to this research will be discussed.

The key factors to consider in thermotropic metallomesogen design are the role of the metal, the design of the ligands and what effect these have on the phase behaviour. Each of these considerations will be examined in the first half of this chapter (Sections 3.2 and 3.3), which the author hopes will provide some context when the reader considers the syntheses performed in this research. In the latter half of the chapter (Sections 3.4 and 3.5 ) the physical properties of metallomesogens will be examined and how they arise from both the chemical structure of the mesogens and their interactions with one another when assembled in the mesophase. ${ }^{20}$ For the most part this will be done by considering key examples from the literature, and examining the physics and chemistry underlying these studies. 


\subsection{The Role of the Metal}

As one might imagine, the metal that is incorporated into a metallomesogen makes a large contribution to the phase behaviour and the physical properties of the mesophases. ${ }^{20}$ The metal is typically incorporated into the core of the mesogen with an anisotropic, rigid organic framework surrounding it. From a structural perspective, this can mean that the metal's coordination geometry provides much of the required structural anisotropy in the core. Common choices for this purpose are nickel(II), copper(II), oxovanadium(IV), palladium(II) and platinum(II). ${ }^{11,21-24}$ Oxovanadium(IV) is an interesting choice as it adopts a non-planar, square pyramidal structure. This is less anisotropic, but the axial oxygen is capable of forming a weak dative bond with the vanadium in a neighbouring mesogen, which often mitigates this problem by stabilising the mesophase. ${ }^{11}$

The use of a metal for its square planar coordination geometry can be problematic in some cases as tetrahedral distortions away from square planar can disrupt the anisotropy of the core and prevent mesophase formation. This is particularly prevalent in nickel(II) complexes, ${ }^{11}$ as was found to be the case early in this study, hence nickel was abandoned in preference for copper. In certain complexes, this problem is solved by modifying the ligand to form a more rigid coordination environment that forces the metals to adopt a planar geometry.

Some interesting examples of mesogens in which the square planar geometry provides the required structural anisotropy in the core are dinuclear ortho-metallated palladium benzylimine and benzylazine complexes (Figure 3.2.1). The choice of bridging ligand is important here, as it can have a marked effect on the nature of the mesophases formed. For example, the use of chloro bridging ligands produces a flat core, and the molecule behaves as a non-chiral calamitic liquid crystal. ${ }^{21}$ If on the other hand, one uses acetate bridging groups, the core is bent, and due to the orientation of the ligands, the molecule is optically active. ${ }^{5}$ With some further modification, optically pure variants of (2) in Figure 3.2.1 have been isolated and demonstrated to exhibit chiral mesophases. ${ }^{5}$ This illustrates the delicate nature of core design since destabilisation of the mesophase occurs upon the formation of a bent core in compound (1) when acetate bridging ligands are used. $^{21}$ 


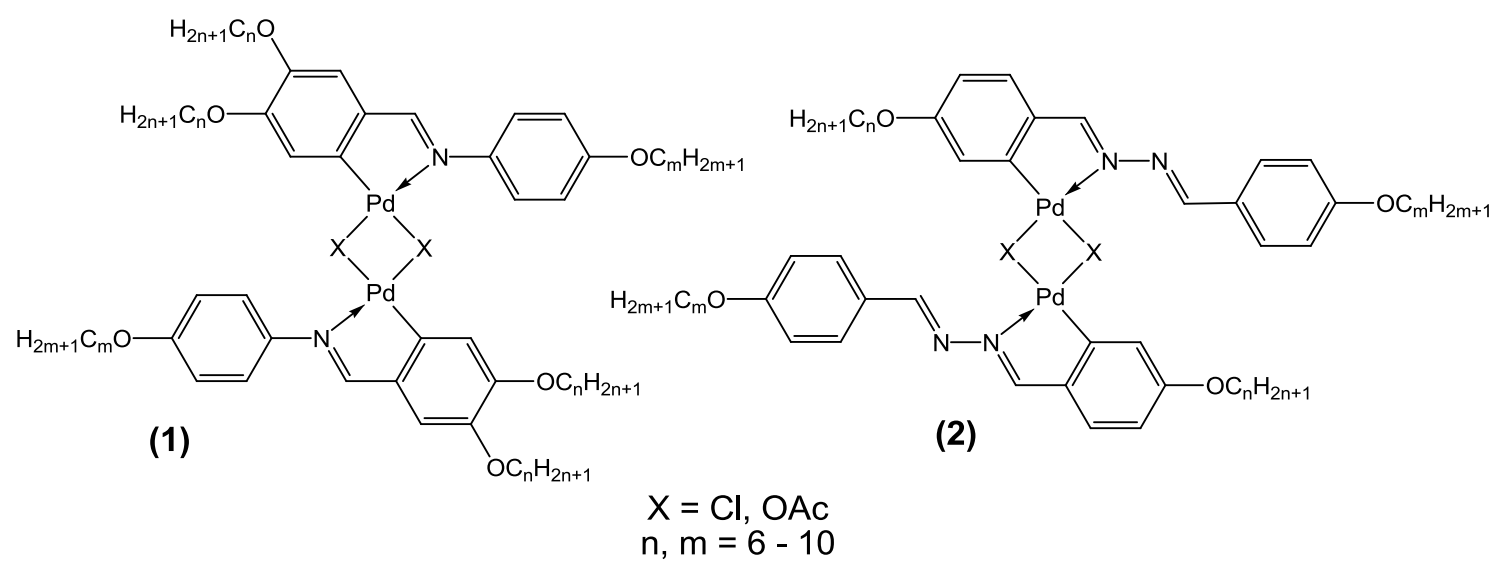

Figure 3.2.1: The mesomorphic series of bridged, dimeric benzylimine (1) and benzylazine (2) palladium complexes.

In addition to their use as a source of structural anisotropy, much of the interest in the inclusion of metals into mesogens arises from the unique physical properties that they may incorporate into the mesophases (see Sections $3.4 \& 3.5) .{ }^{3}$ This is particularly true of lanthanide-based metallomesogens (lanthanidomesogens ${ }^{25}$ ). Lanthanides, unlike the transition metals discussed above, typically form high coordination number complexes, making the design of an anisotropic core challenging. ${ }^{26}$ The key is to bind it to a large ligand that already exhibits mesomorphism. This approach was put forward for the synthesis of lanthanidomesogens by Cardinaels et al., ${ }^{27}$ but had been successfully used previously to incorporate other large, non-anisotropic moieties into mesogens such as silsesquioxanes ${ }^{28}$ and fullerenes. ${ }^{29}$

\subsection{Ligand Design}

The design of the ligands that are employed in a metallomesogen is fundamental in determining whether or not the complex forms mesophases. The principal elements to consider are the size and shape of the core, and the length, positioning and number of the tails. Each of these aspects will be examined in the following sections, as well as the effect their variation has on the phase behaviour of the metallomesogen.

\subsubsection{Size and Shape of the Core}

The design of the core of the mesogen is generally based around two factors: the inclusion of the metal without disrupting the anisotropy of the core and the desired shape of the core (calamitic or discotic). There is a multitude of different core designs that produce different mesophase characteristics, and a selection will be examined here which highlight some of the key considerations in core design. 
Calamitic metallomesogens must incorporate a long, rigid, core in order to form mesophases. ${ }^{5}$ As such, metals that naturally form linear complexes or transsubstituted square planar complexes are ideal for incorporation into calamitic metallomesogens. For this purpose, the group 9, 10 and 11 metals are often employed. ${ }^{5}$ One particular series of metallomesogens discussed by Bruce exploit the linear geometry of silver complexes by complexation with stilbazole ligands ${ }^{30}$ (Figure 3.3.1).

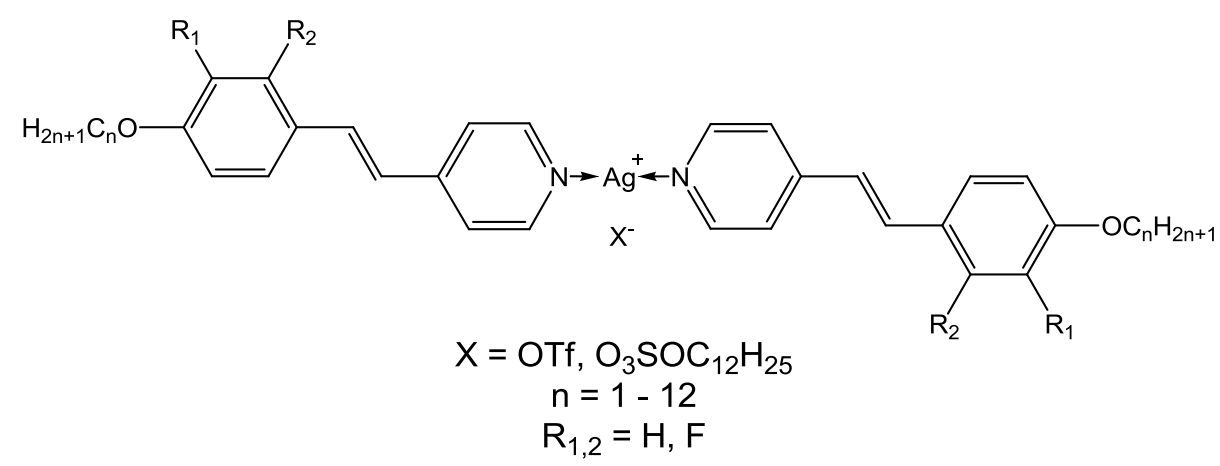

Figure 3.3.1: Mesomorphic stilbazole complexes of silver(I).

An interesting effect noted by Bruce is the consequence of fluorination at either the 2 or 3 positions of the terminal phenyl rings $\left(R_{1}\right.$ and $R_{2}$ respectively in Figure 3.3.1). Substitution at either position has a significant effect on the phase behaviour. Substitution at the 2 position appears to disrupt packing in the smectic A mesophase through lateral steric hindrance. ${ }^{31}$ Substitution at the 3 position, however, appears to stabilise the smectic A mesophase, most likely because in this position the fluorine is less disruptive of packing, and acts to enhance lateral intermolecular attraction, thereby promoting the packing in the smectic A mesophase. ${ }^{31}$ This effect demonstrates the importance of both stabilising interactions, and lateral steric hindrance on mesophase formation.

Another point to note from this study is the effect of lengthening the core. Whilst lengthening the core alone can have a destabilising effect on the mesophases, ${ }^{11}$ when coupled with additional tails, a new type of mesogen called a polycatenar (meaning "many tailed") mesogen is formed. These systems represent an intermediate type of mesogen between calamitic and discotic, the nature of the mesophases that form generally being determined by the number of tails. Hexacatenar (six tails) and pentacatenar (five tails) mesogens tend to form columnar mesophases (discotic behaviour), whereas tricatenar mesogens (three tails) tend to form smectic phases 
(calamitic behaviour). ${ }^{30}$ These systems are examined in more detail later in Section 3.3.2 where the effects of tail length and placement are discussed.

An additional way of incorporating a square planar metal into the core is via the use of Schiff bases. Schiff base-mercury mesogens were first examined by Vorländer in 1923, and represent the earliest known example of metallomesogens. ${ }^{3}$ Of particular interest are the salicylaldimine complexes, examples of which are shown in Figure 3.3.2. The salicylaldimine moiety used to bind the metal is both anisotropic and it allows for easy derivitization to larger, more complex core architectures. A number of these complexes were described in a review by Hoshino, ${ }^{11}$ and some conclusions from that review will be examined when the effects of tail length and location are examined in Section 3.3.2.
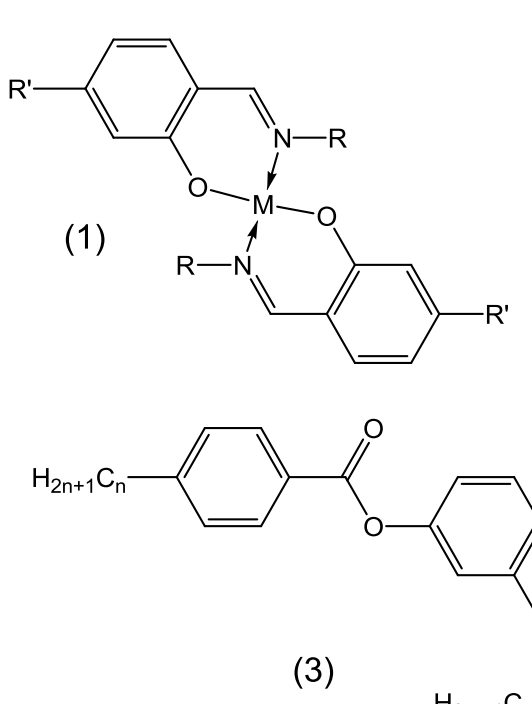

(3)

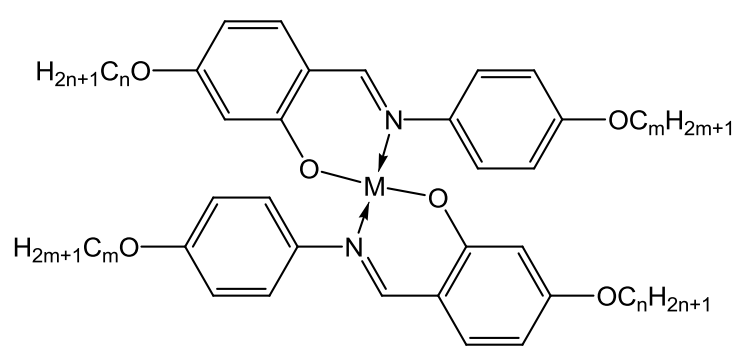

(2)
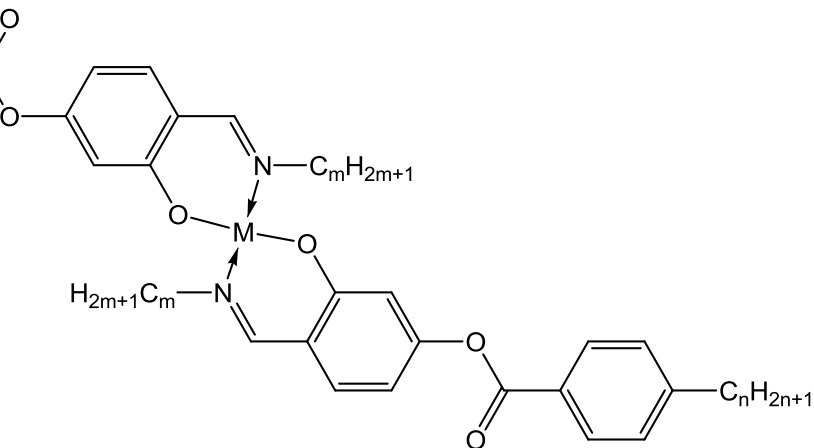

$\mathrm{M}=\mathrm{Ni}, \mathrm{Cu}, \mathrm{Pd}, \mathrm{VO}, \mathrm{FeCl}(\mathrm{III})$

Figure 3.3.2: The archetypical salicylaldimine complex (1) with examples of multi-ring cores (2 and 3) that can be synthesized using this formalism.

A point noted in the review is the importance of the outboard dipole moment arising from ether linkages connecting the tails to the core (as in (2) in Figure 3.3.2) in the formation of the smectic $\mathrm{C}$ mesophase. Dipole interactions also play an important role in stabilising the mesophases formed by "bent core" metallomesogens. These are mesogens in which a rod shaped core is bent in the centre, similar to the shape of a banana. This bent shape means that the core is less anisotropic, but mesophases tend to be stabilised by interactions between permanent dipoles (inherent to the bent 
shape) on the mesogens. ${ }^{11}$ This also has important consequences for the physical properties of the mesophases.

As mentioned in Section 3.2, many of the metals incorporated into metallomesogens do not have an anisotropic coordination geometry. This is often achieved in calamitic mesogens by using a large mesomorphic, ortho-metallating ligand, in conjunction with a number of smaller ligands that fill the remaining coordination sites on the metal. ${ }^{5}$ This strategy has been used successfully to form metallomesogens from octahedral transition metals ${ }^{5}$ (Figure 3.3.3) and lanthanidomesogens. ${ }^{32}$

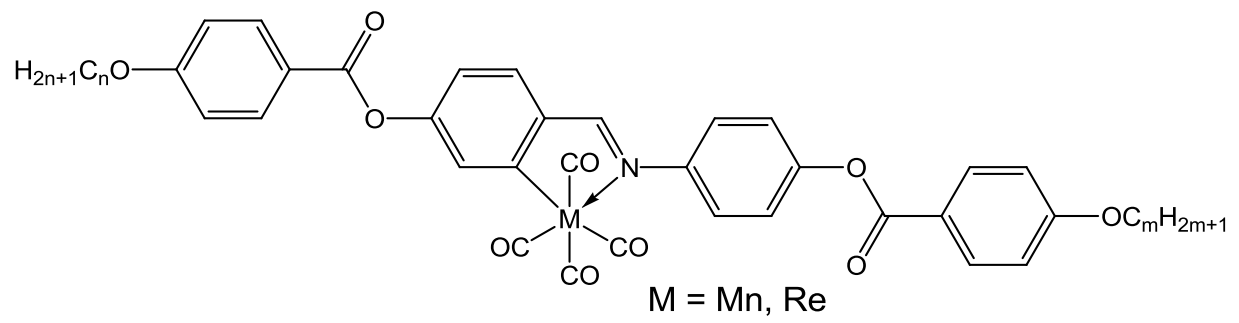

Figure 3.3.3: An example of a metallomesogen incorporating an octahedral transition metal via orthometallation.

Discotic metallomesogens often present less of a design challenge than their calamitic counterparts. ${ }^{5}$ The main goal of discotic mesogen core design is to produce a large, rigid, disc shaped core and surround it with tails in the form of alkyl chains. Incorporation of a metal with a non-anisotropic coordination geometry into such a core can be achieved more easily than in the calamitic case through the use of large chelating ligands, however many discotic metallomesogens do incorporate metals with square planar geometries. These complexes often use the square planar geometry of the metal as a basis to form the flat disc shape needed. Examples of these are the $\beta$-diketonate-based discotic metallomesogens (Figure 3.3.4).

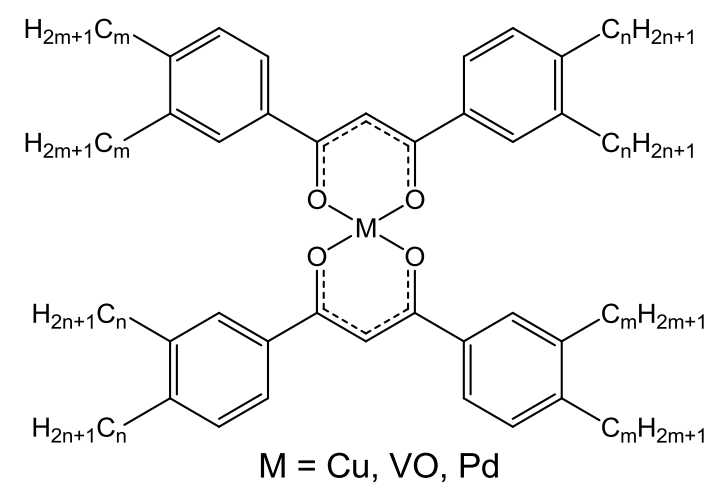

Figure 3.3.4: A series of B-diketonate based discotic metallomesogens.

An interesting series of copper-based discotic metallomesogens are the copper alkanoate "lantern" complexes synthesised by Giroud-Godquin and Marchon, ${ }^{33}$ shown 
in Figure 3.3.5. These mesogens differ from other discotic metallomesogens in two respects. The first is that the size of the "disc" is small compared to other discotic mesogens, and the second is that it has fewer tails than would normally be expected of a discotic metallomesogen ${ }^{5}$ (see Section 3.3.2). The other important observation is that the temperature range of the resulting columnar mesophase is relatively unaffected by changes in the tail length. ${ }^{33}$

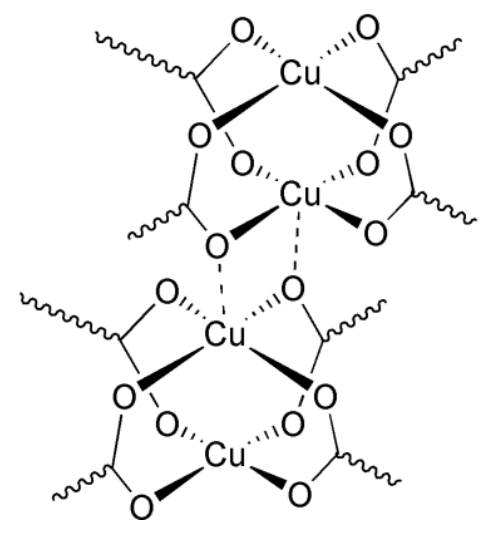

Figure 3.3.5: The repeating unit of the copper alkanoate complexes developed by Giroud-Godquin and Marchon. Image reproduced from Date et al. - "Metallomesogens by Ligand Design".

The reason suggested for these properties is that the columnar mesophase is stabilised by a strong axial intermolecular interaction between each copper atom and an oxygen atom on the adjacent mesogen, forming a "pseudo-polymer" along the column axis ${ }^{5}$ (shown by the dashed lines in Figure 3.3.5). Analogous rhodium, ruthenium, molybdenum and chromium complexes have been synthesised, all of which display similar phase behaviour. ${ }^{20}$

In contrast with the small disc size of the lantern complexes are the discotic metallomesogens incorporating macrocyclic ligands, such as metallo-porphyrins and metallo-phthalocyanines. The columnar mesophases formed by these complexes are usually stable over a wide temperature range, often up to the decomposition temperature of the complex. ${ }^{17}$ The stabilising interaction is thought to arise from $\pi$ stacking interactions between adjacent mesogens in the column. ${ }^{20}$ These macrocyclic ligands are also able to bind to metals with complex, non-anisotropic coordination geometries which makes them ideal for use in lanthanidomesogens (although many lanthanides are too large for the binding site, they will still bind and sit out of the plane of the ligand ${ }^{17}$ ). These forms of discotic metallomesogens are often quite sensitive to tail length and placement, as will be discussed in Section 3.3.2. 
In conclusion, in order to design the core of a metallomesogen, one must take into consideration: the magnitude and origin of any stabilising interactions, the presence of any structural elements that may destabilise the mesophase, the way in which the metal is to be included and if the metal will act to stabilise or destabilise the mesophase through its intermolecular interactions.

\subsubsection{The Location, Number and Length of the Tails}

As mentioned previously, the presence of one or more tails in a mesogen is a very important factor in determining whether or not it is mesomorphic. ${ }^{20}$ Variations in the location and length of the tails, as well as how many there are, have a large effect on the mesophase characteristics of the mesogen. ${ }^{5}$ One might imagine that tail location is less of an issue for calamitic metallomesogens than for discotic metallomesogens. This is certainly true of small, linear metallomesogens in which there are generally no more than two chains. In these complexes, mesophases are generally more stable with increasing tail length, with smectic phases being promoted over nematic phases. ${ }^{30}$ These trends do not necessarily hold, however, when calamitic cores that have lateral chains are employed, or in polycatenar systems.

The effect of lateral tail length of simple calamitic metallomesogens has been studied extensively in salicylaldimine systems, in which the lateral tails are generally those bound to the nitrogen (see Figure 3.3.2). In particular, the review by Hoshino ${ }^{11}$ details the phase behaviour of these complexes. The data suggest that the lateral tails are vital in mesophase formation, as they disrupt packing in an additional dimension to the axial tails. Their lengths (in proportion with the axial tails) can be tuned to promote the formation of the smectic $C$ mesophase over the smectic $A$ mesophase and the nematic mesophase over smectic mesophases. These trends are not universal though, and Hoshino notes that there are more complex interactions than simple sterics that play a part. $^{11}$

As noted in section 3.3.1, polycatenar mesogens exhibit mesophase behaviour that is intermediate between discotic and calamitic. One might consider this odd, as their elongated cores make them appear far more calamitic than discotic; however the origin of the different kinds of mesophase behaviour is actually in the number of, and placement of the tails. ${ }^{5}$ In hexacatenar and pentacatenar systems (c.f. section 3.3.1) the mesophase behaviour is purely discotic, whereas the behaviour of tricatenar 
systems is purely calamitic. ${ }^{34,35}$ However, the behaviour of tetracatenar systems is far more interesting. As shown in Figure 3.3.6, at short tail lengths the mesogens exhibit nematic and smectic mesophases, while at long tail lengths they exhibit columnar mesophases. $^{34,35}$ Studies of the abrupt transition from smectic $\mathrm{C}$ to hexagonal columnar do not show a significant change in local structure. ${ }^{35}$ In addition, it is thought that in the columnar phases, the "discotic layers" in the columns are actually formed from groups of several polycatenar mesogens aligned parallel. ${ }^{5,30,34,35}$

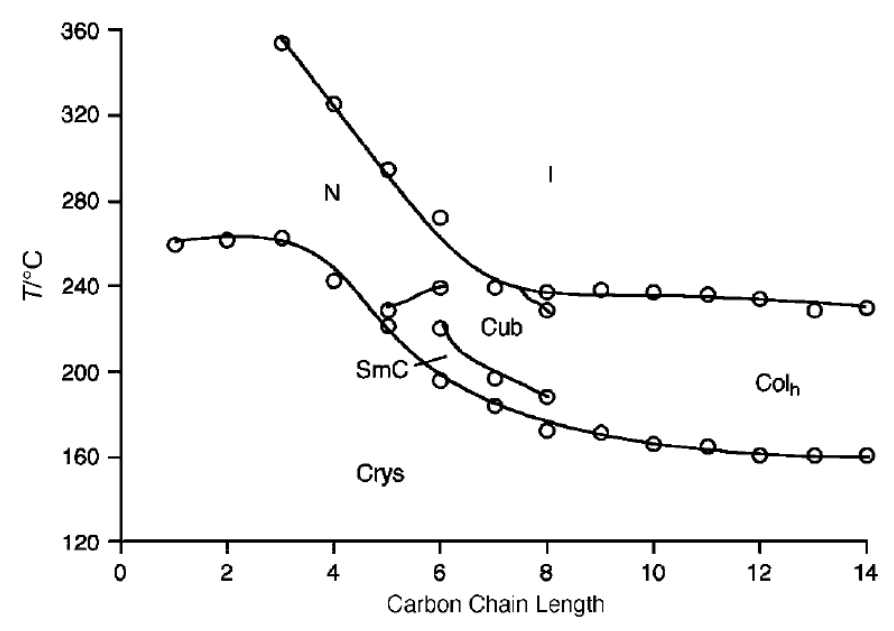

Figure 3.3.6: A phase diagram for a homologous series of tetracatenar bipyridines. Image reproduced from Fazio et al. - "Bending and shaping: cubics, calamitics and columnars". 34

Discotic metallomesogens are also sensitive to the placement, number and length of the tails. Generally speaking, there must be tails fully surrounding the periphery of the disc in order for the complex to be mesomorphic ${ }^{5}$ (although as was noted with polycatenar systems, this is not universally true of mesogens that exhibit columnar mesophases). The relative sizes of the disc and the tails appears to influence whether or not the mesogen is tilted in the column, forming a rectangular mesophase, or a hexagonal mesophase, respectively. ${ }^{17}$

A good example of the sensitivity of discotic metallomesogens to the number of tails is demonstrated by the $\beta$-diketonate-based metallomesogens shown in Figure 3.3.4. It was found that variants with four chains, one on each phenyl ring, were not mesomorphic (regardless of the length of the tails), whereas those with eight were. ${ }^{5,20}$ It was also found on further investigation that discotic mesogens of this size, required at least six tails (of the appropriate length) spaced evenly around the disc in order to be mesomorphic, ${ }^{5}$ but that this number increased as the size of the ring increased. ${ }^{17}$ 
Hence, the notion that the periphery of the disc must be surrounded with tails in order for the complex to be mesomorphic.

\subsection{Electrical and Optical Properties}

The most prevalent commercial application of liquid crystals is, by far, the liquid crystal display, which is now utilised in most forms of digital display technology. ${ }^{36}$ The LCD makes use of the optical response of chiral mesogens in the chiral nematic mesophase when an electric field is applied across them. In "twisted nematic" LCDs, the mesogens respond by "unwinding" their helical alignment (cf. Chapter 2, Section 2.2.1), which changes the way they rotate polarised light. ${ }^{36}$ Metallomesogens are unsuitable for application in this form of LCD due to their generally high melting points and viscosity. This in turn means the applied electric field strength must be high, resulting in the metal ligand bonds on the mesogen being quite labile. ${ }^{3}$ There are, however, several other electrical and optical properties that metallomesogens do exhibit, among these are ferroelectricity and antiferroelectricity, birefringence (Appendix A) and non-linear optical properties. ${ }^{20}$ Whilst interesting in their own right, these properties are not due in any great part to the presence of a metal in the mesogen, and are commonly observed in organic liquid crystals. ${ }^{13,20}$ As such their presence in the mesophases of metallomesogens will not be discussed any further.

\subsection{Luminescent and Electronic Properties}

One of the main reasons that metallomesogens are synthesised and studied is the unique luminescent properties that the transition metal or lanthanide imparts to the mesophase. There are, however, some major difficulties in the study of the luminescence of metallomesogens in the mesophase, the principal problem being the high melting points of metallomesogens. This is because at elevated temperatures, excited states are more likely to decay via non-radiative processes, making their luminescence less visible. ${ }^{37}$ In addition, many of the transitions that are studied in transition metals and lanthanides are weak, spin forbidden $d-d$ and $f-f$ transitions meaning the emission from these transitions is effectively masked at elevated temperatures. ${ }^{25}$ As a result, there have been a number of studies of metallomesogens in the crystalline state and dissolved in organic solvents, ${ }^{22-24,38-40}$ but relatively few of metallomesogens in a mesophase. Vitrification of the mesophases is possible; however 
the rapid rise in viscosity that occurs with rapid cooling can mean that loading the sample into a cuvette for analysis can be extremely difficult. ${ }^{25}$ Recently, though, there has been a number of studies of metallomesogens that are liquid crystalline at room temperature and in the following paragraphs the luminescent properties of several of these metallomesogens will be examined.

An interesting study is that of the hexacatenar iridium(III) complex shown in Figure 3.5.1. The study was conducted on a vitrified columnar mesophase, which curiously was not observed when the sample was slowly cooled. ${ }^{41}$ Upon transition from the isotropic liquid to crystalline state, the phosphorescent emission of the compound changes from a red-orange colour to bright green, however when cooled quickly to form the columnar mesophase, the change is to a bright yellow emission. Moreover, there is a pronounced increase in the phosphorescence quantum yield upon formation of the columnar mesophase, whereas the exact opposite behaviour is observed when the crystalline phase is formed.
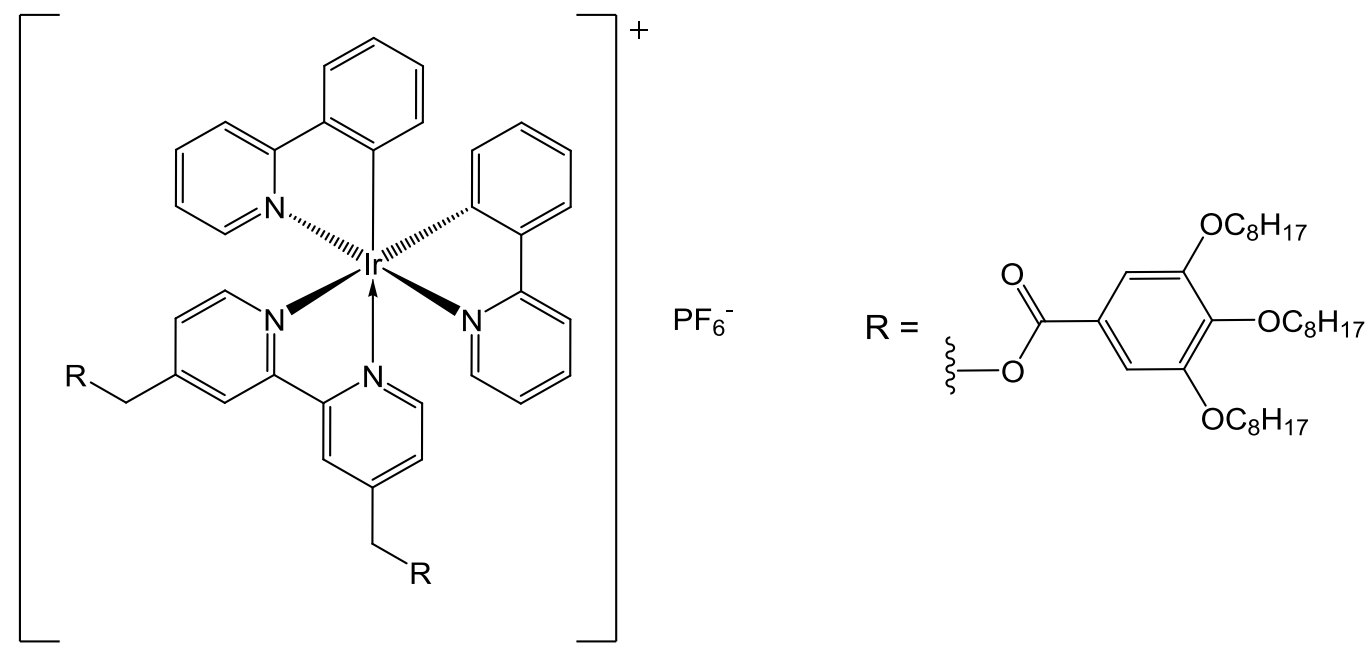

Figure 3.5.1: The hexacatenar iridium(III) metallomesogen which exhibits order driven red/green switching.

This behaviour is due to a phenomenon known as aggregation induced emission (AIE). It has been observed in other iridium(III) complexes of this general structure and as the name suggests, is a direct result of the self assembly of the mesogens in the mesophase. ${ }^{42}$ The principal absorbance of the complex shown in Figure 3.5 .1 results from a triplet metal to ligand charge transfer $\left({ }^{3} \mathrm{MLCT}\right)$, which is a transition that involves a substantial redistribution of charge. ${ }^{41}$ Relaxation back to the ground state occurs readily in the isotropic liquid phase; however this is not the case in the liquid crystalline state, meaning that the emission in the mesophase is often shifted in 
wavelength. This can be for two reasons: the first is that the restriction of rotational motion in the mesophase means that the emissive energy of the ${ }^{3} \mathrm{MLCT}$ is different to that of the complex in the liquid phase and second is due to the possibility of forming an intermolecular excimer state. ${ }^{42}$ The second possibility arises from the packing of the mesogens in the mesophase, which promotes $\pi-\pi$ interactions between the aromatic cores of adjacent mesogens. This enables charge transfer between two adjacent mesogens (known as metal to ligand to ligand charge transfer - MLLCT) or even across several neighbouring mesogens (MLLLCT, MLLLLCT, etc). ${ }^{42}$ This behaviour is not observed in the crystalline phase though, as excited states have a greater tendency to quench via non-radiative processes in crystals due to phonon interactions. ${ }^{18,41}$ This is the proposed reason for the increase in phosphorescence quantum yield upon mesophase formation.

Another interesting study was the one by Sakurai et al. ${ }^{43}$ in which the charge transport properties of certain triply fused porphyrin-copper dimer mesogens (Figure 3.5.2) in the columnar mesophase at room temperature were examined. Both the hetero and the homo mesogens exhibited semiconductor charge transport characteristics, but differed in the transport mechanism. The photocurrent profile of the hetero complex was shown to behave as an n-type semiconductor with an electron mobility of $1.3 \times 10^{-3} \mathrm{~cm}^{2} \mathrm{~V}^{-1} \mathrm{~s}^{-1}$ (cf. $1350 \mathrm{~cm}^{2} \mathrm{~V}^{-1} \mathrm{~s}^{-1}$ in silicon ${ }^{44}$ ). The homo complex (in which the only difference is the number of fluorinated tail moieties) behaves as a p-type semiconductor with a hole mobility of $4.3 \times 10^{-3} \mathrm{~cm}^{2} \mathrm{~V}^{-1} \mathrm{~s}^{-1}$ (cf. $480 \mathrm{~cm}^{2} \mathrm{~V}^{-1} \mathrm{~s}^{-1}$ in silicon ${ }^{44}$ ). This result was also confirmed by transient absorption studies of the homo complex, which showed evidence of the presence of a radical cation species. $^{43}$

Since this change in behaviour results from such a small variation in the structure, it was proposed that the origin of the change is in the self assembly of the mesogens rather than a change of the electronic properties of the individual mesogens. Sakurai et al. suggest that because of the fluorination in the tails, the homo mesogens stack in a twisted geometry in the columns to maximise interactions between the fluorinated tails, and the hetero mesogens stack in a slipped geometry to minimise interactions between the fluorinated and non-fluorinated tails. ${ }^{43}$ This assertion agrees with previous quantum mechanical predictions concerning the effects of altering the $\pi$ - 
stacking geometry on the charge carrier species in semiconducting columnar mesophases. ${ }^{45}$

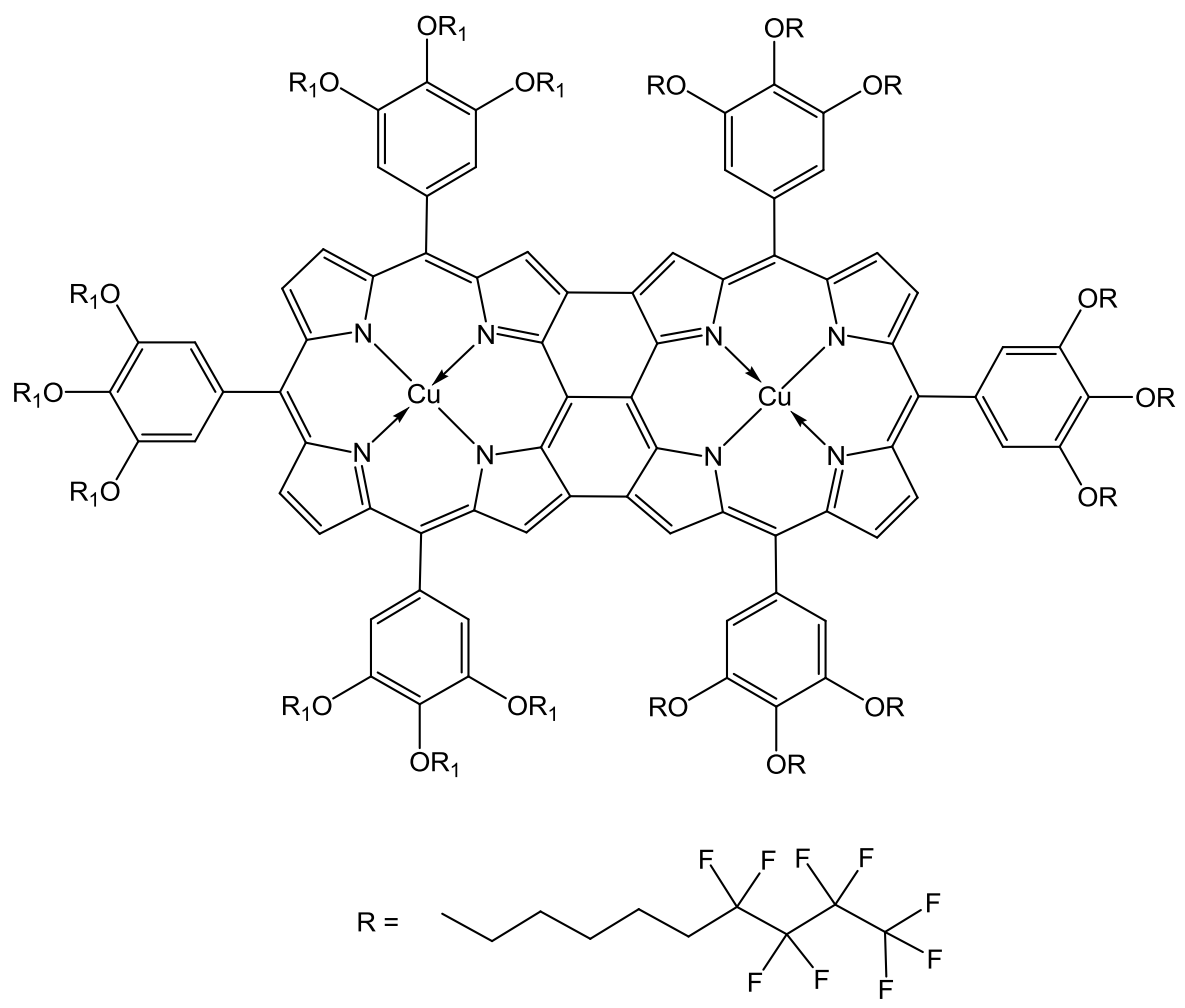

homo: $\mathbf{R}_{\mathbf{1}}=\mathbf{R}$

hetero: $\mathbf{R}_{\mathbf{1}}=$

Figure 3.5.2: The triply fused porphyrin-copper mesogens that exhibit $p$ or $n$ type semiconductor characteristics.

In addition to the studies mentioned above, there is also a significant level of interest in the luminescent properties of lanthanidomesogens. This is because lanthanides have a rich photochemistry, typically exhibiting metal centred luminescence resulting from transitions within the $4 \mathrm{f}$ shell. ${ }^{25}$ Since the emission results from $\mathrm{f}$-shell transitions, the ligands surrounding the metal have little effect on their intensity and fine structure. A drawback to the study of these emissions is that $f-f$ transitions do not satisfy conservation of spin angular momentum and as a result the absorbance of these bands is typically low. ${ }^{25}$ There are techniques to enhance the absorbance of these bands, including the use of strongly absorbing organic ligands which absorb the radiation and then transfer the energy to the metal. This often has an additional benefit in that the presence of large organic ligands around the metal centre can inhibit non-radiative decay processes, which in turn increases the lifetime of the excited state. ${ }^{25}$

Despite there having been relatively few studies undertaken into the luminescence of lanthanidomesogens in the mesophase, ${ }^{25}$ the increasing number of examples of room 
temperature mesomorphic lanthanidomesogens means that it is likely that they will be a source of interest in years to come. Already there is evidence that the metal centred luminescence of europium and terbium based-lanthanidomesogens can be used to probe the transitions between mesophases. ${ }^{46,47}$ In addition, there is also the possibility of doping an organic liquid crystal matrix with an emissive, non mesomorphic lanthanide complex. Whilst this goes beyond the scope of this study, there is some promise in this area, with evidence showing that the liquid crystal host matrix can direct the polarisation of the emission. ${ }^{48}$

One of the potential applications that is often cited for metallomesogens is their use as catalysts in the mesophase. ${ }^{3}$ This is understandable given the ability of transition metals to catalyse a vast number of reactions through the use of their variable oxidation states and coordination geometries. The group 10 metals, which are often used in metallomesogens for their anisotropic coordination sphere, are especially prolific as catalysts (of particular note are the palladium-catalysed cross coupling reactions for which the 2010 Nobel Prize in chemistry was awarded to Suzuki, Negishi and $\mathrm{Heck}^{49}$ ). Given the electronic properties exhibited by metallomesogens in the mesophase, one would imagine that their application in the catalysis of redox electron transfer would be of interest. However, to date, there do not appear to have been any studies in this regard. 


\section{Chapter 4}

\section{Synthesis of Ligands and Complexes}

\subsection{Introduction}

As discussed in Chapter 3, the key design considerations when synthesising a potential metallomesogen are the size and shape of the core, the incorporation of the metal and the quantity and length of tails. The first two aspects are generally interrelated as the metal is almost always incorporated into the core of the mesogen, although there are some exceptions where the coordination site is decoupled from the mesogenic core in order to incorporate metals with highly anisotropic coordination geometries ${ }^{26,27}$ (e.g. lanthanides). One of the most effective ways to incorporate the metal is to choose those metals that have an anisotropic coordination geometry (e.g. square planar or linear group 10 and 11 complexes) and to use rigid, conjugated chelating ligands to encase the metal and thereby form the core. This can be achieved using bidentate Schiff base ligands that ligate via the phenolic oxygen and imine nitrogen as the complexes that are formed tend to be relatively stable, and are generally easily synthesised. ${ }^{11,20}$

The series of complexes investigated here are the copper(II) 4-alkoxysalicylaldimine complexes shown in Figures 4.1.1 and 4.1.2. Analogous group 10 and 11 transition metal salicylaldimine complexes have been shown to be mesogenic. ${ }^{11,50-54}$ In 1998 Hoshino $^{11}$ reviewed the numerous modifications to the salicylaldimine core that have been examined for their influence on the liquid crystalline phase behaviour of the complex. The $\mathrm{N}$-alkyl derivatives $\mathrm{Cu} 1(\mathrm{~m}, \mathrm{n})$, however, were not included in the review. The mesomorphism of two phenyl ring copper-based $\mathrm{N}$-(4-alkylphenyl) derivatives, which were originally investigated by Ghedini et al. in the late $1980 \mathrm{~s}^{55}$ and early 1990s, ${ }^{56}$ were mentioned briefly in Hoshino's review, with Hoshino highlighting their propensity to form a smectic A mesophase. ${ }^{11} \mathrm{An} \mathrm{N}$-(4-butylphenyl) derivative, Cu3(4,6), was investigated here (Figure 4.1.1). 


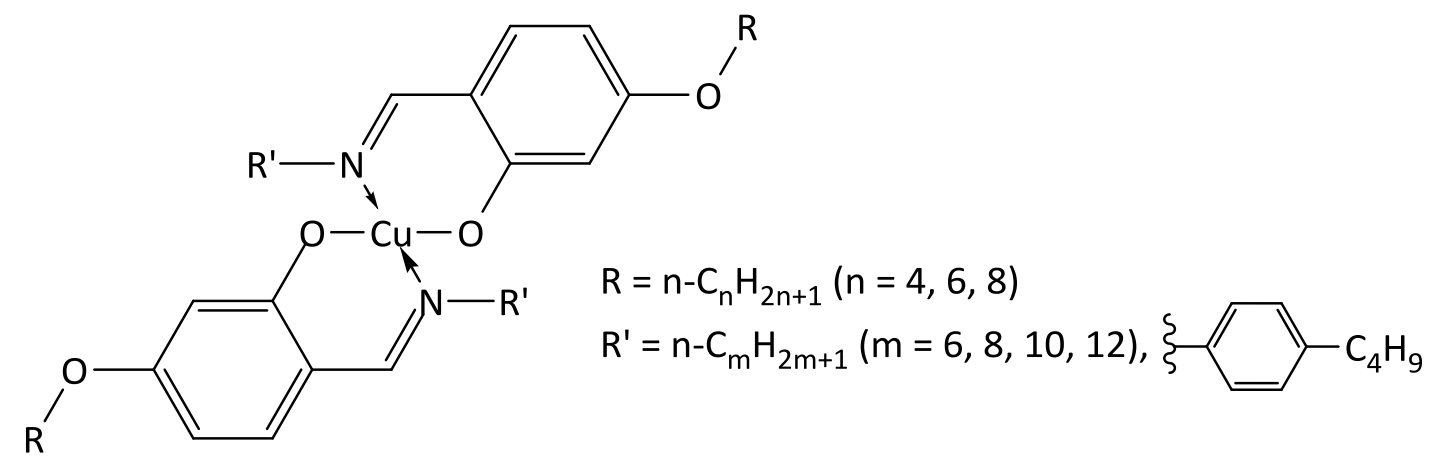

Figure 4.1.1: The mononuclear salicylaldimine complexes $\operatorname{Cu}(m, n)$ and $\mathrm{Cu}(4,6)$.

More recently, Paschke et $a .^{50}$ reported on a study of $\mathrm{N}$-alkyl, copper variants and concluded that they are not mesomorphic based off data obtained from six N-dodecyl and N-octadecyl 4-alkoxysalicylaldimine copper(II) complexes. Paschke et al. also made mention of a family of binuclear copper(II) variants which were mesomorphic and could be easily synthesised from their mononuclear counterparts. Two such complexes were investigated here (Figure 4.1.2).

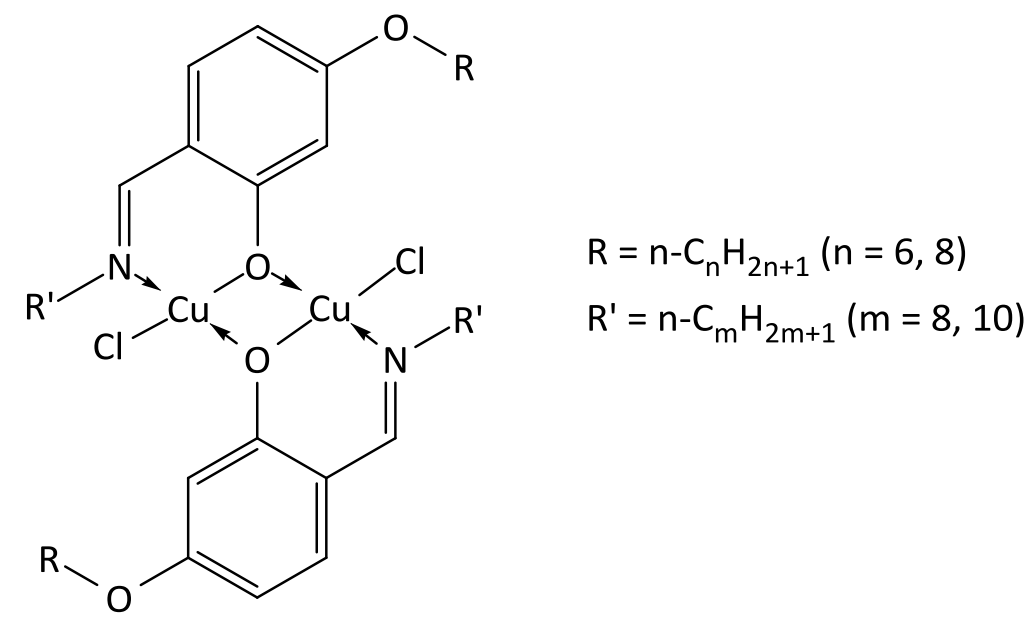

Figure 4.1.2: The binuclear copper salicylaldimine complexes Cu2(m,n).

The methods used in the syntheses in this study are well established in the literature and were adapted from: Yelamaggad et al. (2009), ${ }^{26}$ van Deun and Binnemans (2000), ${ }^{57}$ Paschke et al. (2003), ${ }^{50}$ and López de Murillas (2004). ${ }^{19}$ A general outline of the procedures followed, observations and spectroscopic data are given in Sections 4.2 to 4.4 , in which the synthesis of the ligands, and mononuclear and binuclear complexes will be examined separately. This will be followed by details of the synthetic methods and instruments used in the synthesis portion of the project in Section 4.5. 


\subsection{4-Alkoxysalicylaldimine Ligands}

The salicylaldimine ligands were synthesised in two stages, as shown in Figure 4.2.1. In the first stage, 4-hydroxysalicylaldehyde was reacted with the appropriate straight chain alkyl bromide in the presence of a weak base to selectively form a 4-alkoxysalicylaldehyde. Reaction conditions must be water and oxygen free to avoid decomposition of the remaining 4-hydroxysalicylaldehyde. Any decomposition product is easily separated by flash chromatography using silica gel. The 4-alkoxysalicylaldehyde products are light yellow oils, and were characterised using ${ }^{1} \mathrm{H}$ NMR spectroscopy (Table 4.2.1 and Figure 4.2.2).
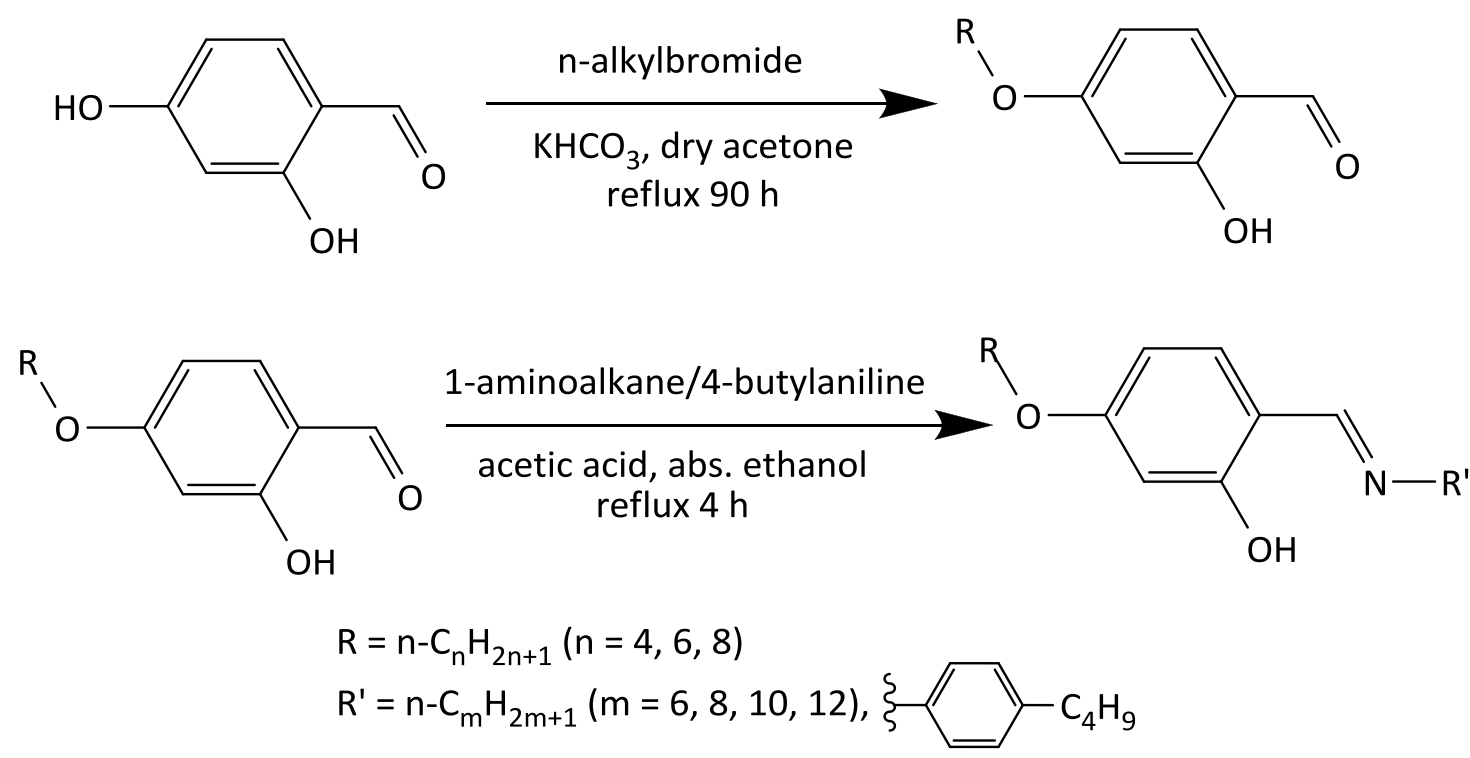

Figure 4.2.1: General synthetic scheme used in the synthesis of the 4-alkoxysalicylaldimine ligands.

The second step is the formation of the imine $\mathrm{C}=\mathrm{N}$ bond via the condensation reaction between the 4-alkoxysalicylaldehyde and an appropriate amine in the presence of a small quantity of acetic acid. The L1 ligands were synthesised using straight chain 1-aminoalkanes, and $L 3(4,6)$ was synthesised using 4-butylaniline. The product is yellow/orange and in some cases (particularly the $L 3(4,6)$ ligand) crystalline at room temperature.

All the ligands are air stable, but fairly moisture sensitive, readily undergoing hydrolysis of the imine $\mathrm{C}=\mathrm{N}$ bond to form an aldehyde and amine. Due to the trace levels of water in most laboratory solvents, the hydrolysis products could not be completely separated using flash chromatography. The acidity of the silica gel used for chromatography may also have catalysed the hydrolysis. 
Table 4.2.1: ${ }^{1}$ H NMR data obtained from the 4-alkoxysalicylaldehydes

\begin{tabular}{ccc}
\hline Environment & $\boldsymbol{\delta}^{\mathbf{1}} \mathbf{H}$ ppm & Multiplicity \\
\hline 1 & 11.48 & $\mathrm{~s}, 1 \mathrm{H}$ \\
2 & 9.70 & $\mathrm{~s}, 1 \mathrm{H}$ \\
3 & 7.41 & $\mathrm{~d}, 8.8 \mathrm{~Hz}, 1 \mathrm{H}$ \\
4 & 6.52 & $\mathrm{dd}, 8.8,2.3 \mathrm{~Hz}, 1 \mathrm{H}$ \\
5 & 6.41 & $\mathrm{~d}, 2.2 \mathrm{~Hz}, 1 \mathrm{H}$ \\
6 & 4.00 & $\mathrm{t}, 6.6 \mathrm{~Hz}, 2 \mathrm{H}$ \\
7 & 1.79 & $\mathrm{qi}, 7.1 \mathrm{~Hz}, 2 \mathrm{H}$ \\
8 & 1.45 & $\mathrm{~m}, 2 \mathrm{H}$ \\
9 & $1.2-1.4$ & $\mathrm{~m},(\mathrm{~m}-4) \mathrm{H}^{*}$ \\
10 & 0.91 & $\mathrm{t}, 7.1 \mathrm{~Hz}, 3 \mathrm{H}$ \\
\hline${ }^{*}$ Note that in the instance where $\mathrm{m}=4$, this peak is absent
\end{tabular}<smiles></smiles>

Figure 4.2.2: The ${ }^{1}$ H NMR environments in the 4-alkoxysalicylaldehydes.

The ligands were characterised by infrared spectroscopy (Table 4.2.2) and a range of NMR techniques (Table 4.2 .3 and Table 4.2.4), including: ${ }^{1} \mathrm{H},{ }^{13} \mathrm{C}$, correlation spectroscopy (COSY), heteronuclear single quantum correlation (HSQC) and heteronuclear multiple bond coherence $(\mathrm{HMBC})$. Of particular note is the shift of the phenolic proton in the ${ }^{1} \mathrm{H}$ NMR spectrum, which is at $11.48 \mathrm{ppm}$ in 4-hexyloxysalicylaldehyde, but at $14.15 \mathrm{ppm}$ in $\mathrm{L} 1(6,6)$. This indicates that the proton is more deshielded in the imine than in the aldehyde and that it forms a stronger intramolecular hydrogen-bond with the imine nitrogen than with the aldehyde oxygen. In addition, the peak is much broader in the imine, which is a result of the quadrupole broadening effect of the ${ }^{15} \mathrm{~N}$ nucleus. ${ }^{58}$ The intramolecular hydrogen-bond has important consequences for the electronic absorption properties of the ligands, which will be expanded upon in Chapter 6 . 
Table 4.2.2: A summary of the key features in the $L 1(6,6)$ and $L 3(4,6)$ FTIR spectra

\begin{tabular}{ccc}
\hline Assignment & $\begin{array}{c}\text { L1(6,6) } \\
\text { Wavenumber } / \mathbf{~ c m}^{-1}\end{array}$ & $\begin{array}{c}\text { L3(4,6) } \\
\text { Wavenumber } / \mathbf{~ c m}^{-1}\end{array}$ \\
\hline $\mathrm{sp}^{3} \mathrm{C}-\mathrm{H}$ stretch & $2855(\mathrm{~s}), 2928(\mathrm{~s}), 2955(\mathrm{~s})$ & $2956(\mathrm{~s}), 2958(\mathrm{~s}), 2859(\mathrm{~s})$ \\
$\mathrm{O}-\mathrm{H}$ stretch & $2800(\mathrm{w}+\mathrm{br})$ & $2750(\mathrm{w}, \mathrm{br})$ \\
$\mathrm{C}=\mathrm{N}$ stretch & $1624(\mathrm{~s})$ & $1618(\mathrm{~s}), 1598(\mathrm{~s})$ \\
$\mathrm{C}-\mathrm{OH}$ stretch & $1514(\mathrm{~m})$ & $1514(\mathrm{~m})$ \\
\hline \multicolumn{3}{c}{$(\mathrm{s})=$ strong, $(w+$ br) $=$ weak and broad, $(\mathrm{m})=$ moderate }
\end{tabular}

Table 4.2.3: NMR data obtained from $L 1(6,6)$

\begin{tabular}{|c|c|c|c|c|c|}
\hline Environment & $\delta^{1} \mathrm{H} / \mathrm{ppm}$ & $\delta{ }^{13} \mathrm{C} / \mathrm{ppm}$ & Multiplicity & COSY & HMBC \\
\hline 1 & 0.9 & 14.0 & $\mathrm{t}, 6.9 \mathrm{~Hz}, 3 \mathrm{H}$ & $3-8$ & 3,5 \\
\hline 2 & 0.91 & 14.0 & $\mathrm{t}, 6.6 \mathrm{~Hz}, 3 \mathrm{H}$ & 3-8 & 4,6 \\
\hline 3 & $1.26-1.48$ & 22.6 & $\mathrm{~m}, 12 \mathrm{H}$ & $9 / 10,1 / 2$ & 5 \\
\hline 4 & $1.26-1.48$ & 22.6 & $\mathrm{~m}, 12 \mathrm{H}$ & $9 / 10,1 / 2$ & 6 \\
\hline 5 & $1.26-1.48$ & 31.6 & $\mathrm{~m}, 12 \mathrm{H}$ & $9 / 10,1 / 2$ & 3 \\
\hline 6 & $1.26-1.48$ & 31.5 & $\mathrm{~m}, 12 \mathrm{H}$ & $9 / 10,1 / 2$ & 4 \\
\hline 7 & $1.26-1.48$ & 26.7 & $\mathrm{~m}, 12 \mathrm{H}$ & $9 / 10,1 / 2$ & 5 \\
\hline 8 & $1.26-1.48$ & 25.7 & $\mathrm{~m}, 12 \mathrm{H}$ & $9 / 10,1 / 2$ & 6 \\
\hline 9 & 1.67 & 30.8 & qi, $7.1 \mathrm{~Hz}, 2 \mathrm{H}$ & $11,3-8$ & $5,7,11$ \\
\hline 10 & 1.78 & 29.0 & qi, $7.1 \mathrm{~Hz}, 2 \mathrm{H}$ & $12,3-8$ & $6,8,12$ \\
\hline 11 & 3.52 & 57.1 & $\mathrm{t}, 6.8 \mathrm{~Hz}, 2 \mathrm{H}$ & 9 & 7,9 \\
\hline 12 & 3.96 & 68.0 & $\mathrm{t}, 6.6 \mathrm{~Hz}, 2 \mathrm{H}$ & 10 & 8,10 \\
\hline 13 & 8.09 & 167.7 & $\mathrm{~s}, 1 \mathrm{H}$ & 11 & $11,15,18$ \\
\hline 14 & - & 163.5 & - & - & - \\
\hline 15 & - & 111.8 & - & - & - \\
\hline 16 & 6.33 & 106.7 & $\begin{array}{l}\mathrm{dd}, 8.7 \mathrm{~Hz}, \\
2.3 \mathrm{~Hz}, 1 \mathrm{H}\end{array}$ & 18 & 18,19 \\
\hline 17 & - & 163.1 & - & - & - \\
\hline 18 & 7.06 & 132.5 & $\mathrm{~d}, 8.5 \mathrm{~Hz}, 1 \mathrm{H}$ & 16 & $13,14,17$ \\
\hline 19 & 6.37 & 102.0 & $\mathrm{~d}, 2.2 \mathrm{~Hz}, 1 \mathrm{H}$ & - & $\begin{array}{c}13,14,15 \\
16,17\end{array}$ \\
\hline 20 & 14.15 & - & $\mathrm{s}, 1 \mathrm{H}$ & - & - \\
\hline
\end{tabular}

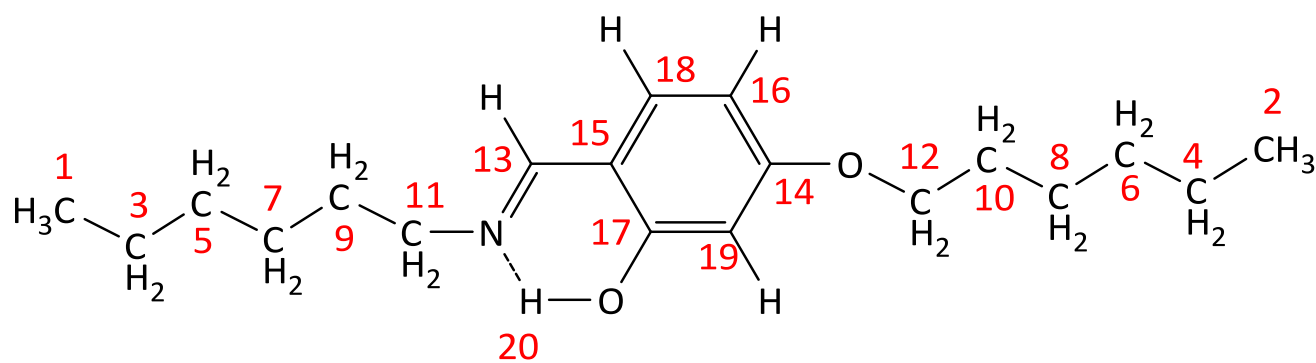

Figure 4.2.3: The assignment of NMR environments $\left({ }^{1} \mathrm{H}\right.$ and/or $\left.{ }^{13} \mathrm{C}\right)$ in $L 1(6,6)$. 
Table 4.2.4: NMR data obtained from $L 3(4,6)$

\begin{tabular}{cccccc}
\hline Environment & $\boldsymbol{\delta}^{\mathbf{1}} \mathbf{H}$ ppm & $\boldsymbol{\delta}^{\mathbf{1 3}} \mathbf{C} / \mathbf{p p m}$ & Multiplicity & COSY & HMBC \\
\hline 1 & 0.92 & 14.0 & $\mathrm{t}, 7.0 \mathrm{~Hz}, 3 \mathrm{H}$ & $3-6$ & 6,4 \\
2 & 0.94 & 14.0 & $\mathrm{t}, 7.6 \mathrm{~Hz}, 3 \mathrm{H}$ & $3-6$ & 9,3 \\
3 & $1.3-1.5$ & 22.3 & $\mathrm{~m}, 8 \mathrm{H}$ & $1 / 2,7 / 8$ & 8,2 \\
4 & $1.3-1.5$ & 22.6 & $\mathrm{~m}, 8 \mathrm{H}$ & $1 / 2,7 / 8$ & 6,1 \\
5 & $1.3-1.5$ & 25.7 & $\mathrm{~m}, 8 \mathrm{H}$ & $1 / 2,7 / 8$ & 6 \\
6 & $1.3-1.5$ & 31.6 & $\mathrm{~m}, 8 \mathrm{H}$ & $1 / 2,7 / 8$ & - \\
7 & 1.80 & 29.0 & $\mathrm{qi}, 7.5 \mathrm{~Hz}, 2 \mathrm{H}$ & $10,3-6$ & $10,6,5$ \\
8 & 1.61 & 33.7 & $\mathrm{qi}, 7.6 \mathrm{~Hz}, 2 \mathrm{H}$ & $9,3-6$ & 9,3 \\
9 & 2.63 & 35.2 & $\mathrm{t}, 7.8 \mathrm{~Hz}, 2 \mathrm{H}$ & 8 & $17,15,8,3$ \\
10 & 4.00 & 68.2 & $\mathrm{t}, 6.6 \mathrm{~Hz}, 2 \mathrm{H}$ & 7 & $20,7,5$ \\
11 & 6.49 & 101.6 & $\mathrm{~s}, 1 \mathrm{H}$ & - & $21,20,13,12$ \\
12 & 6.47 & 107.5 & $\mathrm{~d}, 8.5 \mathrm{~Hz}, 1 \mathrm{H}$ & 16 & 13 \\
13 & - & 113.0 & - & - & - \\
14 & 7.21 & 120.8 & $\mathrm{~d}, 8.5 \mathrm{~Hz}, 2 \mathrm{H}$ & 15 & 18,15 \\
15 & 7.18 & 129.3 & $\mathrm{~d}, 8.0 \mathrm{~Hz}, 2 \mathrm{H}$ & 14 & 17,14 \\
16 & 7.24 & 133.3 & $\mathrm{~d}, 8.3 \mathrm{~Hz}, 1 \mathrm{H}$ & 12 & 20,19 \\
17 & - & 141.3 & - & - & - \\
18 & - & 145.9 & - & - & - \\
19 & 8.52 & 160.6 & $\mathrm{~s}, 1 \mathrm{H}$ & - & $21,18,16,13$, \\
20 & - & 163.5 & - & - & 12 \\
21 & - & 164 & - & - & - \\
22 & 13.94 & - & $\mathrm{s}, 1 \mathrm{H}$ & - & - \\
\hline
\end{tabular}

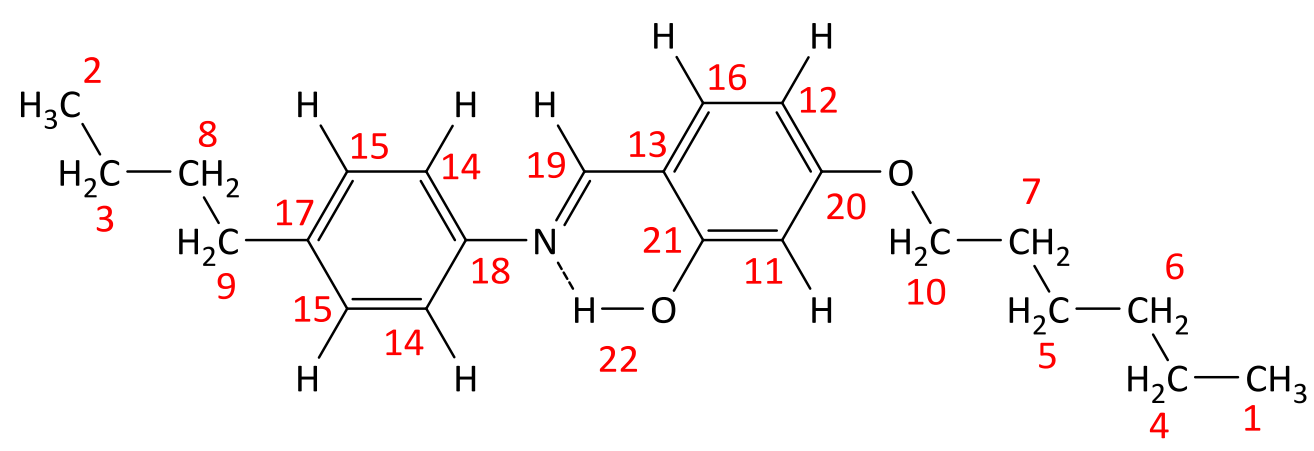

Figure 4.2.4: The assignment of NMR environments $\left({ }^{1} H\right.$ and/or $\left.{ }^{13} C\right)$ in $L 3(4,6)$.

\subsection{Mononuclear Complexes}

Complexation of the 4-alkoxysalicylaldimine ligands with copper(II) was achieved in a single step by combining the ligand with copper(II) acetate in absolute ethanol (Figure 4.3.1). The nine copper complexes synthesised are crystalline, appearing either fibrous 
or film like, and either golden or yellow-green in colour. An example of the $\mathrm{N}$-alkyl complexes, $\mathrm{Cu} 1(8,8)$, is shown in Figure 4.3.2.

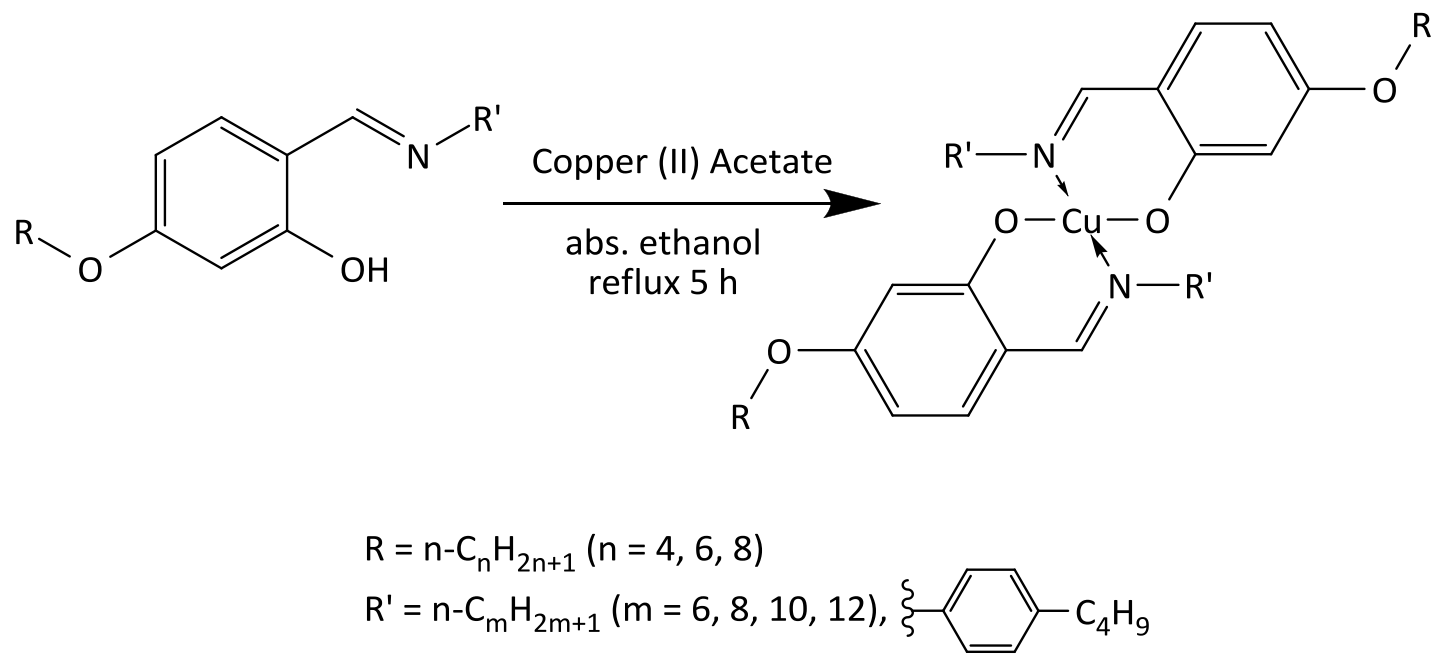

Figure 4.3.1: The complexation of the 4-alkoxysalicylaldimine ligand $L 1(m, n)$ and $L 3(4,6)$ to a transition metal to form the $\mathrm{M} 1(m, n)$ and $\mathrm{Cu} 3(4,6)$ complexes.

The complexes were characterised by infrared spectroscopy (Table 4.3.1). The imine $\mathrm{C}=\mathrm{N}$ stretching frequency is particularly sensitive to complexation. In the L1 ligand spectra, the frequency is approximately $1625 \mathrm{~cm}^{-1}$ (see Table 4.2.2), but this shifts to lower wavenumber by between $5 \mathrm{~cm}^{-1}$ and $25 \mathrm{~cm}^{-1}$ upon complexation. This indicates a weakening of the bond resulting from a decrease in electron density brought about by the Lewis basicity of the imine nitrogen. The $L 3(4,6)$ IR spectrum had two strongly absorbing bands in the $1600 \mathrm{~cm}^{-1}$ to $1620 \mathrm{~cm}^{-1}$ range which both shift to lower frequency by $10 \mathrm{~cm}^{-1}$ upon complexation, meaning they are most likely two different stretching modes of the $\mathrm{C}=\mathrm{N}$ bond.

Table 4.3.1: A summary of the key features in the Cu1(6,6) and Cu3(4,6) FTIR spectra

\begin{tabular}{ccc}
\hline Assignment & $\begin{array}{c}\text { Cu1(6,6) } \\
\text { Wavenumber } / \mathbf{~ c m}^{-1}\end{array}$ & $\begin{array}{c}\text { Cu3(4,6) } \\
\text { Wavenumber } / \mathbf{~ c m}^{-1}\end{array}$ \\
\hline $\mathrm{sp}^{3} \mathrm{C}-\mathrm{H}$ stretch & $2957(\mathrm{~s}), 2932(\mathrm{~s}), 2857(\mathrm{~s})$ & $2954(\mathrm{~s}), 2931(\mathrm{~s}), 2858(\mathrm{~s})$ \\
$\mathrm{C}=\mathrm{N}$ stretch & $1605(\mathrm{~s})$ & $1608(\mathrm{~s}), 1587(\mathrm{~s})$ \\
$\mathrm{C}-\mathrm{OM}$ stretch & $1527(\mathrm{~s})$ & $1523(\mathrm{~m})$ \\
\hline & $(\mathrm{s})=$ strong and $(\mathrm{m})=$ moderate
\end{tabular}

This pattern of decreasing $\mathrm{C}=\mathrm{N}$ stretching frequency upon complexation of Schiff bases has been observed in a number of studies of salicylaldimine-transition metal complexes, ${ }^{53,57,59,60}$ and was extensively studied by Kovacic. ${ }^{61}$ In addition, the broad phenol $\mathrm{O}-\mathrm{H}$ stretch between $2300 \mathrm{~cm}^{-1}$ and $3300 \mathrm{~cm}^{-1}$ in the ligand spectra (the 
extreme breadth and low frequency are most likely due to the intramolecular hydrogen-bond ${ }^{60}$ ) is absent in the complex spectra.

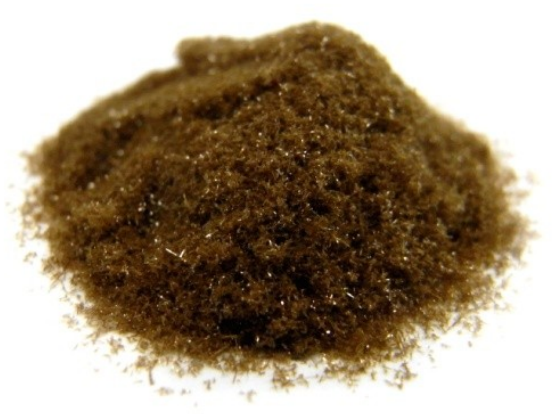

Figure 4.3.2: The Cu1(8,8) complex.

Elemental analysis was used to characterise $\operatorname{Cu} 1(6,6)$ and $\operatorname{Cu}(4,6)$, the results of which agreed completely with predicted values (see Table 4.3.2). In addition, crystal structures were obtained for $\mathrm{Cu1}(8,6)$ and $\mathrm{Cu} 1(6,6)$; these will be discussed in Chapter 5.

Table 4.3.2: The elemental analysis results for $\mathrm{Cu1}(6,6)$ and $\mathrm{Cu3}(4,6)$. The results are given as mass percentages with the predicted values in parentheses

\begin{tabular}{|c|cc|} 
Complex & $\mathrm{Cu} 1(6,6)$ & $\mathrm{Cu}(4,6)$ \\
Formula & $\mathrm{CuC}_{38} \mathrm{H}_{60} \mathrm{~N}_{2} \mathrm{O}_{4}$ & $\mathrm{CuC}_{46} \mathrm{H}_{60} \mathrm{~N}_{2} \mathrm{O}_{4}$ \\
$\mathbf{C}$ & $68.18 \%(67.87 \%)$ & $72.32 \%(71.89 \%)$ \\
$\mathbf{H}$ & $9.31 \%(8.99 \%)$ & $8.01 \%(7.87 \%)$ \\
$\mathbf{N}$ & $4.20 \%(4.17 \%)$ & $3.65 \%(3.65 \%)$ \\
$\mathbf{C u}$ & Not analysed & $8.3 \%(8.27 \%)$
\end{tabular}

\subsection{Binuclear Complexes}

The binuclear complexes were synthesised in a single step from the corresponding mononuclear complexes, as shown in Figure 4.4.1. The reaction proceeds quickly at first, as evidenced by a colour change when the reagents are mixed to form a deep red solution, but then the reaction mixture must be left to stand at room temperature for 24 to 48 hours to ensure the reaction is completed. Paschke et al. ${ }^{50}$ proposed that this observation is due to the rate limiting step being the formation of the $\mathrm{Cu}-\mathrm{Cl}$ bonds in which the chloride anions must displace coordinated solvent molecules (ethanol in this case). They explain the initial colour change as being due to the rapid formation of a bisethanolodicopper(II) complex, and then crystallisation of the product occurs slowly as the coordinated ethanol ligands are displaced by chloride anions. The product forms dark red crystals. 
<smiles></smiles>

Figure 4.4.1: The synthesis of the binuclear copper(II) complexes by reaction with copper(II) chloride.

Cu2 $(10,6)$ was characterised by elemental analysis (which matched the predicted values - see Table 4.4.1) and infrared spectroscopy (Table 4.4.2). The C-O stretch in the infrared spectrum is of particular note as the complexation of the additional copper causes a shift to higher frequency $\left(1530 \mathrm{~cm}^{-1}\right.$ to $\left.1550 \mathrm{~cm}^{-1}\right)$, most likely due to the movement of the oxygen being constrained by the additional copper. This effect is also noted in previous studies of these systems. ${ }^{50}$ No such effect is apparent in the $\mathrm{C}=\mathrm{N}$ stretching frequency (which remains at $1618 \mathrm{~cm}^{-1}$ ), suggesting that the interaction of the nitrogen with the copper is unaffected by the complexation of an additional copper atom.

Table 4.4.1: The elemental analysis results for Cu2(10,6). The results are given as mass percentages with the predicted values in parentheses

\begin{tabular}{|c|c|} 
Complex & $\mathrm{Cu2(10,6)}$ \\
Formula & $\mathrm{C}_{46} \mathrm{H}_{76} \mathrm{Cl}_{2} \mathrm{Cu}_{2} \mathrm{~N}_{2} \mathrm{O}_{4}$ \\
$\mathbf{C}$ & $60.38 \%(60.11 \%)$ \\
$\mathbf{H}$ & $8.46 \%(8.33 \%)$ \\
$\mathbf{N}$ & $3.01 \%(3.05 \%)$ \\
$\mathbf{C l}$ & $7.54 \%(7.71 \%)$ \\
$\mathbf{C u}$ & $14.1 \%(13.83 \%)$
\end{tabular}


Table 4.4.2: A summary of the key features in the Cu1(10,6) (for comparison) and Cu2(10,6) FTIR spectra

\begin{tabular}{ccc}
\hline Assignment & $\begin{array}{c}\text { Cu1(10,6) } \\
\text { Wavenumber } / \mathbf{~ c m}^{-\mathbf{1}}\end{array}$ & $\begin{array}{c}\text { Cu2(10,6) } \\
\text { Wavenumber } / \mathbf{~ c m}^{-\mathbf{1}}\end{array}$ \\
\hline $\mathrm{sp}^{3}$ C-H stretch & $2958(\mathrm{~s}), 2925(\mathrm{~s}), 2854(\mathrm{~s})$ & $2958(\mathrm{~s}), 2925(\mathrm{~s}), 2854(\mathrm{~s})$ \\
$\mathrm{C}=\mathrm{N}$ stretch & $1619(\mathrm{~s})$ & $1620(\mathrm{~s})$ \\
C-OM stretch & $1530(\mathrm{~m})$ & $1552(\mathrm{~m})$ \\
\hline
\end{tabular}

$(s)=$ strong and $(m)=$ moderate

\subsection{Experimental}

Syntheses done under inert atmospheres were carried out by degassing the reaction vessel in nitrogen gas and then fitting the vessel with a balloon of the relevant gas (all supplied by BOC New Zealand). NMR spectra were obtained in deuterated chloroform (Sigma Aldrich, 99.8\% atom D, 17.6 ppm $\mathrm{H}_{2} \mathrm{O}$ - stored over $4 \AA$ molecular sieves) in a Varian Unity Inova 500 spectrometer with ${ }^{1} \mathrm{H}$ resonance at $500 \mathrm{MHz}$ and ${ }^{13} \mathrm{C}$ at $125 \mathrm{MHz}$. Infrared spectra were obtained on a Bruker Tensor 27 FTIR spectrometer in attenuated total reflectance (ATR) mode and elemental analysis was carried out by the Campbell Microanalytical Laboratory at the University of Otago. In all other aspects common laboratory practises were followed.

\subsubsection{4-alkoxysalicylaldimine Ligands}

\section{4-alkoxysalicylaldehydes}

2,4-dihydroxybenzaldehyde (1.08 g, $7.77 \mathrm{mmol}$, Sigma Aldrich, 98\%) was dissolved in dry acetone $\left(10 \mathrm{~mL}\right.$, Panreac, $\geq 99 \%, \leq 0.01 \% \mathrm{H}_{2} \mathrm{O}$, stored under nitrogen over $4 \AA$ molecular sieves) along with potassium bicarbonate $(789 \mathrm{mg}, 7.88 \mathrm{mmol}$, May and Baker Ltd., $\geq 98 \%)$. In a separate flask, the appropriate $\mathrm{n}$-alkylbromide $(7.80 \mathrm{mmol}$, see Table 4.5.1) was dissolved in dry acetone $(10 \mathrm{~mL})$. The two solutions were combined and refluxed under argon for 90 hours while being monitored by thin layer chromatography (4:1 petroleum ether (Pure Science, drum solvent) - ethyl acetate (Panreac, 99.9\%), $254 \mathrm{~nm}$ UV). The product spot has a retention factor of about 0.6, while the 2,4-dihydroxybenzaldehyde is 0.2 . An additional spot appears past 90 hours at an RF of 0.7 , which is believed to be the di-substituted by-product. As the reactions proceeded, a white/pale pink precipitate (thought to be potassium bromide) 
developed and the solution turned yellow/orange in colour. A deep black precipitate was produced in the event of decomposition.

Once complete, the reaction mixtures were filtered through diatomaceous earth (Betta-crop). Silica gel (1 to $2 \mathrm{~mL}$, Pure Science, $40-63 \mu \mathrm{m}$ ) was added and the solvent was removed in vacuo. The crude products were then purified by flash chromatography using a silica gel column that was eluted with mixtures of petroleum ether and ethyl acetate. The 4-alkoxysalicylaldehyde comes off in the 19:1 petroleum ether - ethyl acetate fractions. The purified products were viscous yellow oils and were stored under nitrogen. Yields varied between $20 \%$ and $70 \%$ depending on the amount of decomposition, and how far the reaction had progressed when it occurred.

Table 4.5.1: A summary of the alkylbromides used in the synthesis of the 4-alkoxysalicylaldhydes

\begin{tabular}{cccc}
\hline Ligand Series & Alkylbromide & Supplier & Purity \\
\hline $\mathrm{L} 1(4, n)$ & 1-bromobutane & Merck & $98 \%$ \\
$\mathrm{~L} 1(6, n)$ & 1-bromohexane & Merck & $99 \%$ \\
$\mathrm{~L} 1(8, n)$ & 1-bromooctane & Hopkin and Williams Ltd & $98 \%$ \\
\hline
\end{tabular}

\section{4-alkoxysalicylaldimines}

The appropriate 4-alkoxysalicylaldehyde was dissolved absolute ethanol ( $15 \mathrm{~mL}$, Pure Science, $\geq 98.85 \%$ ). In a separate flask, acetic acid (one equivalent, Pure Science, AR grade - glacial) was dissolved in absolute ethanol $(15 \mathrm{~mL})$ with either a 1-aminoalkane in the case of the $\mathrm{N}$-alkyl L1 ligands (1.2 equivalent, see Table 4.5.2), or 4-butylaniline (1.2 equivalent, Sigma Aldrich, $\geq 97 \%$ ) in the case of the $\mathrm{N}$-(4-butylphenyl) $L 3(4,6)$ ligand. The contents of the second flask were added to the first under nitrogen, resulting in the formation of a deep orange solution. The solution was then refluxed for four hours under nitrogen resulting in a deepening of the orange colour. Once complete (as judged by TLC with the same conditions as previously stated above - the product RF is 0.5 ), the solvent was removed in vacuo, resulting in a dark orange viscous oil.

Table 4.5.2: A summary of the aminoalkanes used in the synthesis of the L1 ligands

\begin{tabular}{cccc}
\hline Ligand Series & 1-aminoalkane & Supplier & Purity \\
\hline $\mathrm{L} 1(m, 6)$ & 1-aminohexane & Merck & $98 \%$ \\
$\mathrm{~L} 1(m, 8)$ & 1-aminooctane & Merck & $98 \%$ \\
$\mathrm{~L} 1(m, 10)$ & 1-aminodecane & Sigma Aldrich & $98 \%$ \\
$\mathrm{~L} 1(m, 12)$ & 1-aminododecane & Sigma Aldrich & $98 \%$ \\
\hline
\end{tabular}


The crude product was purified by flash chromatography using a column of silica gel that was eluted with mixtures of petroleum ether and ethyl acetate. The 4-alkoxysalicylaldimine came off in the 9:1 petroleum ether - ethyl acetate fractions. The purified product was a yellow, viscous oil in the case of the L1 ligands and a yellow crystalline solid that melts slightly above room temperature to form a cloudy yellow fluid that goes clear at higher temperatures in the case of $L 3(4,6)$. Overall yields were generally between $75 \%$ and $85 \%$.

\subsubsection{Mononuclear complexes}

The appropriate ligand $(2.0 \mathrm{mmol})$ was dissolved in absolute ethanol $(10 \mathrm{~mL})$ to form a bright yellow solution. In a separate flask, copper(II) acetate monohydrate $(1.0 \mathrm{mmol}$, May and Baker Ltd., $\geq 98.5 \%$ ) was also dissolved in absolute ethanol (10 mL) to form a deep blue solution. The ligand solution was slowly added to the copper(II) solution whilst it was stirred at $60^{\circ} \mathrm{C}$, resulting in the formation of a very dark green, almost black solution in the case of the Cu1 complexes, and an extremely dark red solution in the case of $\mathrm{Cu} 3(4,6)$. The solution was then refluxed for five hours before being allowed to cool to room temperature overnight, during which time a yellow-green solid formed. Following this, the solvent was removed in vacuo and the resulting green solid was dissolved in dichloromethane (Panreac, $\geq 99.9 \%$ ) and the resulting green solution was filtered through diatomaceous earth. The dichloromethane was then removed in vacuo and the green-brown solid that remained was recrystalised from absolute ethanol. Overall yields were between $60 \%$ and $80 \%$.

The crystals varied considerably between samples. The Cu1(n,6), Cu1(n,8) and Cu3 $(4,6)$ complexes were all small needle shaped or film-like golden-brown crystals (Figure 4.3.2). The Cu1(n,4) complexes, in contrast, all formed green, fibrous crystals. Despite this apparent colour difference in the crystals, there were no significant differences between their solution phase UV-visible absorption spectra (see Chapter 6).

\subsubsection{Binuclear complexes}

Absolute ethanol $(2 \mathrm{~mL})$ was added to a flask containing $\mathrm{Cu} 1(10,6)$ or $\operatorname{Cu}(8,8)$ (0.12 mmol) as appropriate. The mixture was stirred and chloroform (Panreac, $\geq 99.0 \%$ ) was added dropwise until the complex dissolved to form a very dark yellow/green solution. In a separate flask, copper(II) chloride dihydrate $(0.18 \mathrm{mmol}$, 1.5 equiv., BDH Chemicals, 98.0\%) was dissolved in a minimum amount of absolute 
ethanol to give a bright green solution. This was added to the Cu1 solution while stirring, resulting in the immediate formation of a deep red solution, which was then refluxed for 15 minutes. Following this the solution was reduced to half its volume and left to stand for 48 hours.

After 24 hours small, red needle shaped crystals began to form. These were recovered after 48 hours had elapsed and washed with cold absolute ethanol. Overall yields of the final, dried product were around $30 \%$. 


\section{Chapter 5}

\section{Structure and Phase Behaviour}

\subsection{Introduction}

Metallomesogens and liquid crystals in general, have fascinated researchers for over a century ${ }^{20}$ because of their combination of long-range order and fluidity. This seemingly paradoxical combination is a result of a number of different factors (as discussed in Chapter 2), including the type and magnitude of interactions between the mesogens, the shape of the cores of the mesogens and the conformational entropy of the tail moieties. Each of these aspects is determined by the chemical structure of the mesogens, meaning that the challenge for liquid crystal researchers has always been to find relationships between the design of the mesogens and their phase behaviour.

Although still not completely understood, the aspects of molecular design of organic mesogens that result in mesophase formation have been extensively researched over the last four decades. ${ }^{4}$ This means that a substantial body of knowledge now exists on the relationship between chemical structure and mesomorphism of organic mesogens. ${ }^{4,12,13,36}$ However, this is not the case for metallomesogens. The introduction of a metal, particularly a large transition metal, poses a number of design challenges (as discussed in Chapter 3), and introduces different architectures and intermolecular interactions compared with organic mesogens. The best illustration of this comes from the copper alkanoate "lantern" complexes synthesised by Giroud-Godquin et al. in the 1980s and 90s, ${ }^{33}$ which were discussed in Chapter 3. An analogous organic system could not be mesomorphic as its core is small and not particularly rigid, however the strong axial interactions of the copper atoms with the oxygens in adjacent molecules leads to the formation of columnar phases.

Attempts have been made to catalogue the behaviour of certain classes of metallomesogens. The phase behaviour of the classes of salicylaldimine complexes synthesised in this project are particularly well documented in Hoshino's 1998 review, ${ }^{11}$ but Hoshino acknowledges that there is still not a clear understanding of the relationships between chemical structure and mesophase formation of 
metallomesogens. Developing an understanding of this relationship is essential in order to control the self assembly. In this chapter the phase behaviour of the complexes and ligands will be examined with the aim of finding relationships between their structures and phase behaviour.

\subsection{Analysis Techniques and Instrumentation}

\subsubsection{Differential Scanning Calorimetry (DSC)}

With differential scanning calorimetry the difference in heat flow into a sample and an inert reference as a function of raising or lowering the temperature is measured. It is used to monitor changes in the sample which occur due to changes in temperature, such as phase changes. Measurement of the differential heat flow allows precise determination of the temperature of phase transitions. DSC can be used quantitatively to determine the heat of a transition. DSC is used extensively in liquid crystal research to find the temperatures and enthalpies of phase transitions with extreme precision. ${ }^{4,20,26,54}$

A Shimadzu DSC-60, calibrated with metallic indium supplied by Mettler-Toledo $(\leq 200$ ppb impurities), was used. Samples were analysed in crimped aluminium pans (supplied by Shimadzu) in a nitrogen atmosphere, and the data processed using Shimadzu TA-60 thermal data analysis software.

Further information on DSC can be found in Appendix A.

\subsubsection{Polarised Optical Microscopy (POM)}

Polarised optical microscopy (POM) is a technique that relies on the birefringence of a mesophase to produce optical textures that can be used to identify that mesophase. The textures arise due to the behaviour of the director field around disclinations (line defects). The orientation of the director field relative to the two polarising filters (the polariser and the analyser - see Appendix A) determines the strength of the birefringence, so the intensity of the birefringence provides information on the orientation of the director field around a disclination. The behaviour of the director field around disclinations varies between mesophases, as does the texture observed. This is useful to liquid crystal researchers as it provides an easy means by which a substance can be checked for mesomorphism, and it also provides information about the structures present in the mesophase. 
A Nikon Optiphot-2 Pol polarising microscope, fitted with a Nikon D200 DSLR 10.3 megapixel camera was used. Samples were prepared by placing a small quantity (no more than a few milligrams) on a glass slide under a cover slip. The temperature of the samples was controlled using a Mettler-Toledo FP82HT hot stage.

Further information on POM and its application to liquid crystals can be found in Appendix A.

\subsubsection{X-ray Scattering Techniques}

Three different X-ray scattering techniques were used: small angle X-ray scattering (SAXS), variable temperature powder X-ray diffraction (PXRD) and single crystal X-ray crystallography. SAXS can be used to obtain structural information on the $1 \mathrm{~nm}-$ $100 \mathrm{~nm}$ length scale ${ }^{62}$ and so yields information on structural changes that occur upon mesophase formation and destruction, in addition to allowing symmetry information to be obtained. SAXS is superior to POM and DSC in the sense that it gives the most structural information, but it requires larger samples which means that the transition temperatures are invariably less accurate than DSC. In addition, containment of fluid samples can be problematic as samples must be analysed in a vacuum to minimise unwanted scattering of the primary beam. ${ }^{62}$ Here, quartz capillary cells were used for low viscosity fluids at low temperatures, and air tight metal cells with amorphous polymer windows at high temperatures.

PXRD and single crystal X-ray crystallography collect scattering data at wider angles, and hence examine smaller repeat distances than SAXS. Single crystal X-ray crystallography can be used to determine the precise crystal structure of a sample by measuring the scattering of X-rays off a single crystal of the sample. However, the need for a single-domain crystalline sample means that single crystal X-ray crystallography is impractical for many materials. PXRD, in which scattering off a finely ground sample is measured, does not have such restrictions, but provides much less structural information. Scattering off a single crystal results in points which can be correlated to specific planes in the crystal lattice by their position relative to the orientation of the crystal. Scattering off a powder results in the points being averaged out into rings due to the large number of randomly oriented crystallites in the sample. Consequentially, the PXRD data can give information about the symmetry and repeat distances in the sample on the $0.1 \mathrm{~nm}$ to $2 \mathrm{~nm}$ length scale but cannot readily be used 
to determine an exact structure. Here, single crystal X-ray crystallography was used to determine the structure of some complexes in the crystalline phase, while PXRD was used to monitor changes in the crystal structure as a function of temperature.

X-ray crystallography and variable temperature PXRD measurements were performed at Massey University on a Rigaku Spider diffractometer fitted with an Oxford X-Stream temperature device. A slurry of the sample powder and super glue was used for PXRD measurements. Crystal structures were refined using SHELXL- $97^{63}$ by Dr Shane Telfer at Massey University. SAXS measurements were carried out using an Anton-Paar SAXSesS system fitted with a CCD on a line-collimated beamline and phosphor plates (Cyclone Plus phosphor storage system supplied by Perkin-Elmer) on both point and line collimated beamlines allowing small/wide angle measurements to be obtained. Data obtained using the SAXSess system was normalised to the residual primary beam intensity and corrected for dark current and background scattering using the Anton-Paar SAXSquant software package. Copper $\mathrm{K}_{\alpha}$ radiation with a wavelength of $0.1542 \mathrm{~nm}$ was used for all measurements.

\subsection{Mononuclear N-alkyl Salicylaldimine Complexes Cu1}

\subsubsection{Phase Behaviour}

DSC data obtained from $\mathrm{Cu}(8,8)$ are shown in Figure 5.3.2. The data show two transitions when the sample is heated, but only a single transition when the sample is cooled. Although multiple phase transitions are observed, this is not consistent with mesophase formation as mesophases commonly form when cooled from the isotropic liquid phase (monotropic mesophases). ${ }^{4,20}$ It is more likely that this behaviour is the result of two crystalline phases. All of the Cu1 complexes (Figure 5.3.1) showed these two transitions on heating.

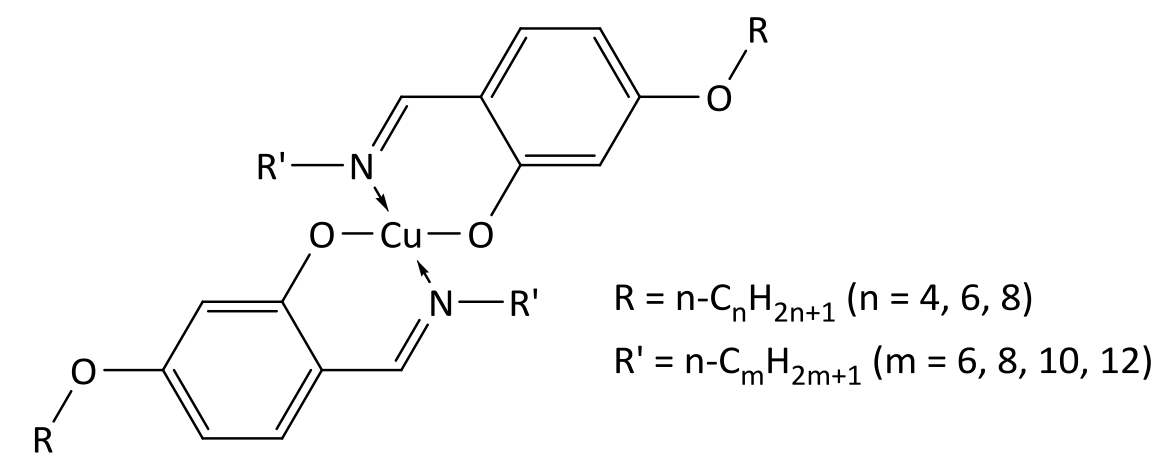

Figure 5.3.1: The structure of the Cu1 complexes. 
The formation of two crystalline phases was confirmed from POM observations (Figure 5.3.3). The two crystalline phases will be referred to as $\mathrm{Cr}_{1}$ and $\mathrm{Cr}_{2}$ (low and high temperature phases respectively). The DSC traces of Cu1(12,4) and Cu1 $(10,4)$ complexes showed an additional transition below the $\mathrm{Cr}_{1}-\mathrm{Cr}_{2}$ transition which was present in both heating and cooling, indicating the presence of another crystal phase at lower temperatures (referred to as $\mathrm{Cr}_{0}$ ). This transition was not observed in any other complex and was not investigated further.

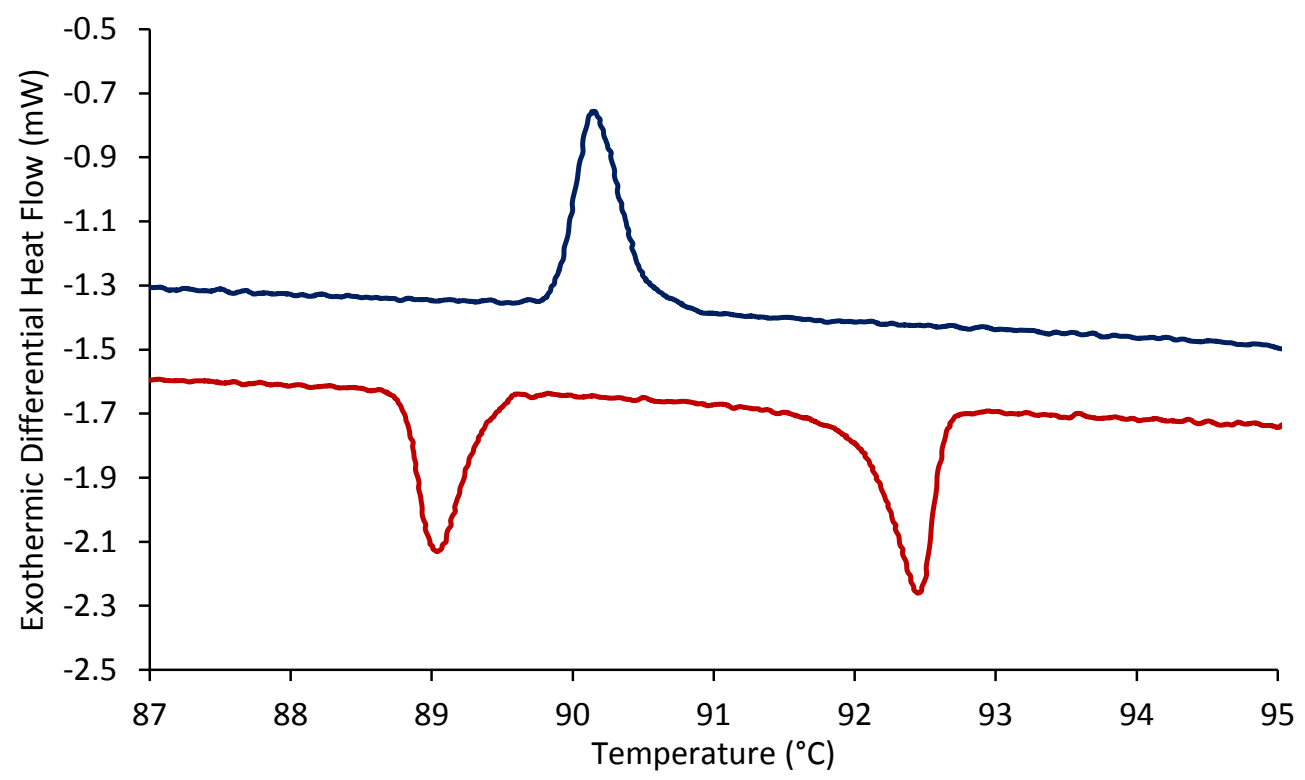

Figure 5.3.2: DSC trace of Cu1(8,8) showing two phase transitions on the heating curve (red) and one on the cooling curve (blue). The scan rate used was $\pm 0.5^{\circ} \mathrm{C} \mathrm{min}^{-1}$. The structure of $\mathrm{Cu1}(8,8)$ is shown for reference.

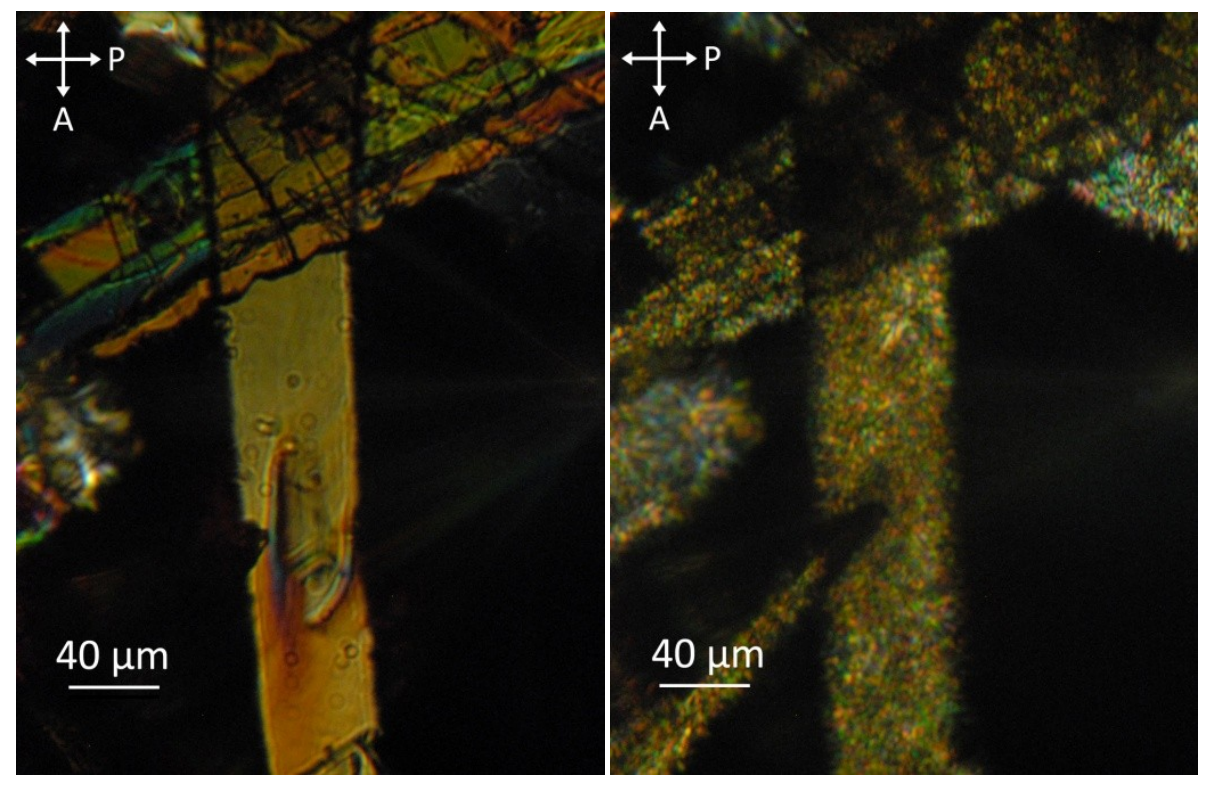

Figure 5.3.3: $P O M$ images of $\mathrm{Cu} 1(8,6)$ in $\mathrm{Cr}_{1}$ at $81.3^{\circ} \mathrm{C}$ (left) and in $\mathrm{Cr}_{2}$ at $85.0^{\circ} \mathrm{C}$ (right). The polariser (P) and analyser (A) orientations are shown. 
Eight different Cu1 complexes were synthesised with a variety of $\mathrm{N}$-alkyl (m) and alkoxy $(n)$ chain lengths. Varying the lengths of the chains had a significant effect on the crystal phase transition temperatures (Figure 5.3.4), particularly the $\mathrm{Cr}_{1}-\mathrm{Cr}_{2}$ transition, occurring between $40^{\circ} \mathrm{C}$ and $95^{\circ} \mathrm{C}$. The use of shorter alkoxy chains was particularly effective at lowering the $\mathrm{Cr}_{1}-\mathrm{Cr}_{2}$ transition, with the butyloxy complexes having transition temperatures up to $60^{\circ} \mathrm{C}$ below the equivalent hexyloxy and octyloxy complexes. The melting point remains relatively unaffected by structural changes to the alkyl chains, only decreasing slightly with increasing $\mathrm{N}$-alkyl chain length.

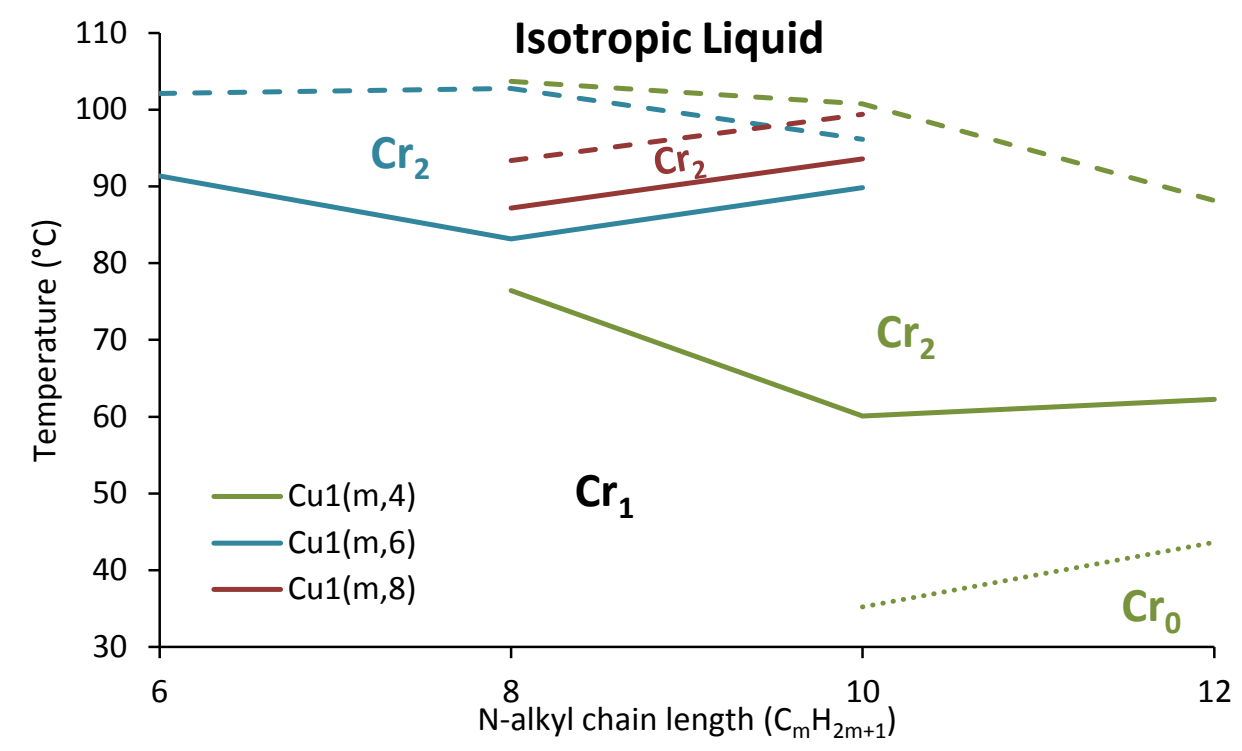

Figure 5.3.4: Phase diagram illustrating the variation of the crystal phase transition temperatures (upon heating) of the Cu1 $(m, n)$ complexes for different $\mathrm{N}$-alkyl $(\mathrm{m})$ and alkoxy $(n)$ chain lengths. The dotted line represents the $\mathrm{Cr}_{0^{-}}-\mathrm{Cr}_{1}$ transition, the solid line represents the $\mathrm{Cr}_{1}-\mathrm{Cr}_{2}$ transition, and the dashed line the melting point.

Despite having a strong influence on the temperatures of the crystal phase transitions, varying the alkyl chain lengths has little effect on the enthalpies of the transitions (Table 5.3.1), aside from an increase in the $\mathrm{Cr}_{1}-\mathrm{Cr}_{2}$ enthalpy in the $\mathrm{N}$-decyl complexes as the length of the alkoxy chain is increased. This indicates that the variation in temperature of the $\mathrm{Cr}_{1}-\mathrm{Cr}_{2}$ transition is not due to changes in the latent heat of the phase transition.

Upon cooling from the isotropic liquid, (in almost all cases) one transition with an enthalpy matching the enthalpy of fusion seen on heating is observed. This indicates that the complexes crystallise into the $\mathrm{Cr}_{2}$ phase. This is supported by repeated heating of the sample immediately after cooling, with only one transition (melting) being observed (Figure 5.3.5). 
Table 5.3.1: The enthalpies of the two phase transitions observed upon heating of the Cu1 complexes

\begin{tabular}{ccc}
\hline Complex & $\mathrm{Cr}_{1}-\mathrm{Cr}_{2} / \mathrm{kJ} \mathrm{mol}^{-1}$ & $\mathrm{Cr}_{2}-\mathrm{L} / \mathrm{kJ} \mathrm{mol}^{-1}$ \\
\hline $\mathrm{Cu} 1(8,8)$ & $-24.4 \pm 0.8$ & $-34.2 \pm 0.8$ \\
$\mathrm{Cu} 1(10,8)$ & $-22.0 \pm 0.8$ & $-37.8 \pm 0.8$ \\
$\mathrm{Cu} 1(6,6)$ & $-26.1 \pm 0.7$ & $-34.9 \pm 0.7$ \\
$\mathrm{Cu} 1(8,6)$ & $-28.6 \pm 0.7$ & $-44.3 \pm 0.7$ \\
$\mathrm{Cu} 1(10,6)$ & $-17.0 \pm 0.8$ & $-32.7 \pm 0.8$ \\
$\mathrm{Cu} 1(8,4)$ & $-24.2 \pm 0.7$ & $-37.6 \pm 0.7$ \\
$\operatorname{Cu} 1(10,4)$ & $-13.1 \pm 0.7$ & $-39.4 \pm 0.7$ \\
$\operatorname{Cu} 1(12,4)$ & $-18.3 \pm 0.8$ & $-45.7 \pm 0.8$ \\
\hline
\end{tabular}

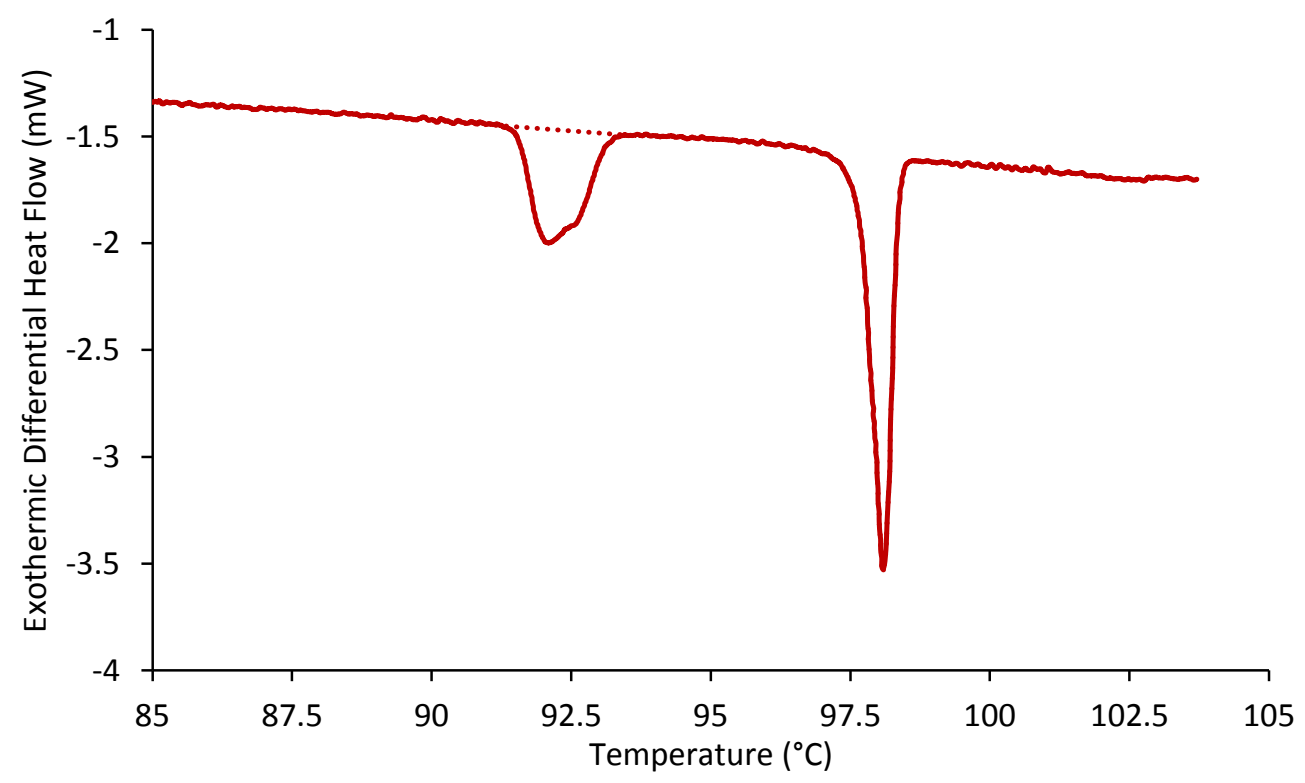

Figure 5.3.5: DSC heating data measured for complex Cu1(10,8) at $0.5^{\circ} \mathrm{C} \mathrm{min}^{-1}$. The solid line is the first heating scan, the dotted line is the second. The shape of the peak suggests the possibility of multiple processes taking place during the $\mathrm{Cr}_{1}-\mathrm{Cr}_{2}$ transition.

Reformation of the $\mathrm{Cr}_{1}$ phase does not typically occur when the complexes are cooled below their $\mathrm{Cr}_{1}-\mathrm{Cr}_{2}$ transition temperatures (except for $\mathrm{Cu1}(12,4)$ and $\left.\mathrm{Cu}(10,4)\right)$ unless the sample is left to stand for long periods of time at temperatures below the transition temperature, or extremely slow cooling rates are used (ca. $-5^{\circ} \mathrm{Chr}$ ). This indicates that the kinetics of phase relaxation in these complexes are not dictated solely by the equilibrium energetics. Furthermore, the shape of the peak on the heating curve indicates multiple processes are taking place during the $\mathrm{Cr}_{1}-\mathrm{Cr}_{2}$ transition (Figure 5.3.5). The complexes' $\mathrm{Cr}_{2}-\mathrm{Cr}_{1}$ phase relaxation behaviour will be discussed in more depth in Section 5.3.3. 


\subsubsection{Structural Variation of the Cu1 Complexes with Temperature}

Single crystals of $\operatorname{Cu} 1(6,6)$ and $\operatorname{Cu} 1(8,6)$ were obtained in sufficient quality for single crystal X-ray crystallography. The crystals were prepared by dissolving the complex in a mixture of ethanol and dichloromethane and allowing slow evaporation of the dichloromethane.

Cu1 $(6,6)$ crystallised in the monoclinic $P 2_{1} / c$ space group with an inversion centre on the copper atom. The copper centre is in a square planar geometry (Figure 5.3.6). In order to satisfy the $90^{\circ}$ bond angle the core is not perfectly flat (Table 5.3.2). The alkoxy chains lie in the plane of the core. The $\mathrm{N}$-alkyl chains are inclined to the plane, with a dihedral angle along the $\mathrm{N}(1)-\mathrm{C}(6)$ bond of $112^{\circ}$ (Figure 5.3.6). $\mathrm{C}(2)$ is disordered with $51 \%$ occupancy in the $(2 \mathrm{a})$ site and $49 \%$ in the $(2 \mathrm{~b})$ site. The crystallographic data are given in Table 5.3.4.
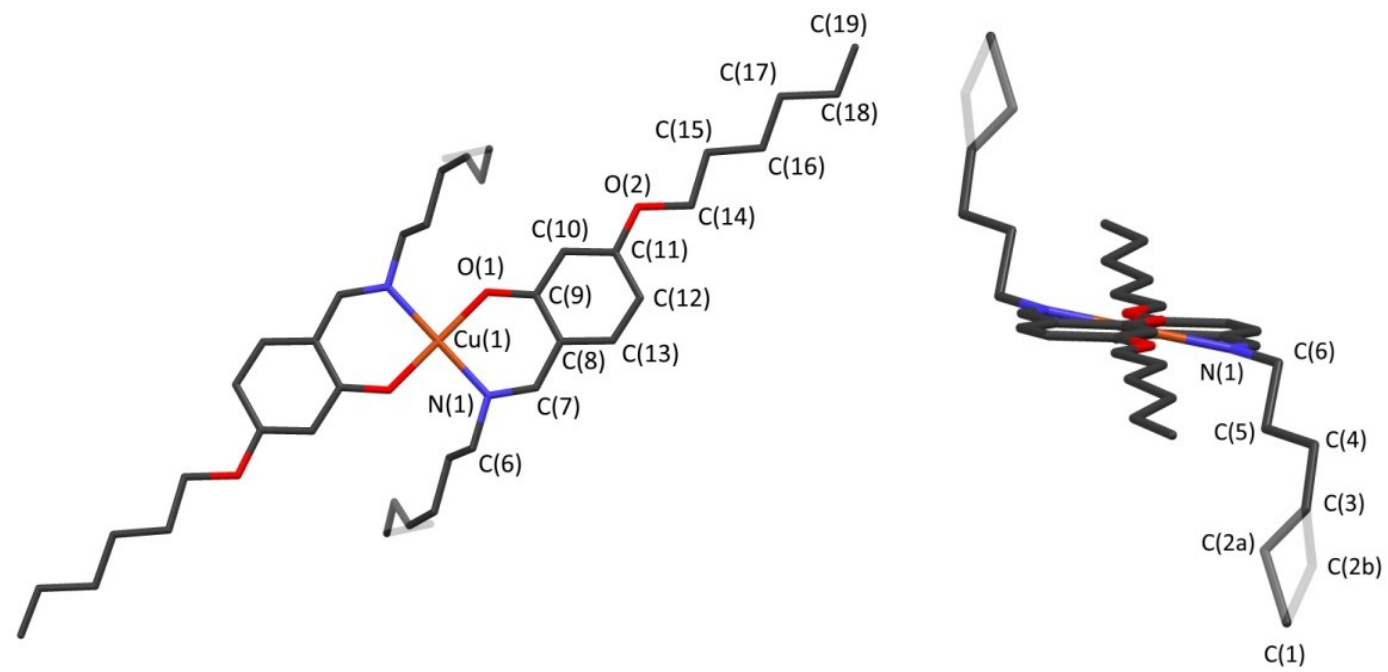

Figure 5.3.6: The structure of Cu1(6,6) as determined by single crystal X-ray crystallography viewed perpendicular (left) and parallel (right) to the core. Note that as there is an inversion centre at $\mathrm{Cu}(1)$, the atoms in each ligand are equivalent and are only labelled once. The hydrogens in the structure have been omitted for clarity.

Table 5.3.2: A selection of bond lengths, bond angles and dihedral angles in the structure of Cu1(6,6)

\begin{tabular}{cccc}
\hline & Length / $\AA$ & & Angle / $^{\circ}$ \\
\hline $\mathrm{Cu}(1)-\mathrm{O}(1)$ & 1.890 & $\mathrm{~N}(1)-\mathrm{Cu}(\mathbf{1})-\mathrm{O}(1)$ & 91.2 \\
$\mathrm{Cu}(1)-\mathrm{N}(1)$ & 2.000 & $\mathrm{C}(7)-\mathrm{N}(1)-\mathrm{Cu}(1)$ & 122.9 \\
$\mathrm{C}(9)-\mathrm{O}(1)$ & 1.320 & $\mathrm{C}(9)-\mathrm{O}(1)-\mathrm{Cu}(1)$ & 129.3 \\
$\mathrm{C}(7)-\mathrm{N}(1)$ & 1.301 & $\mathrm{C}(8)-\mathrm{C}(9)-\mathrm{O}(1)-\mathrm{Cu}(1)$ & 16.4 \\
& & $\mathrm{C}(7)-\mathrm{N}(1)-\mathrm{C}(6)-\mathrm{C}(5)$ & 112.1 \\
\hline
\end{tabular}

The atoms in bold are the central atom in the case of bond angles, or the bond along which the angle is measured in the case of dihedral angles. 
Cu1 $(8,6)$ crystallises in the triclinic $P \overline{1}$ space group with two crystallographicallyindependent (albeit identical) molecules (Figure 5.3.7(insert)). As with Cu1(6,6) the copper adopts a square planar geometry and the core deviates slightly from being perfectly flat (Figure 5.3.7). Also like $\mathrm{Cu}(6,6)$ the $\mathrm{N}$-alkyl chains are inclined to the plane of the core with a similar dihedral angle along the $N(1)-C(8)$ bond of $111^{\circ}$. The crystal structure is shown in Figure 5.3.7 with selected bond lengths and angles in Table 5.3.3. The crystallographic data are given in Table 5.3.4.

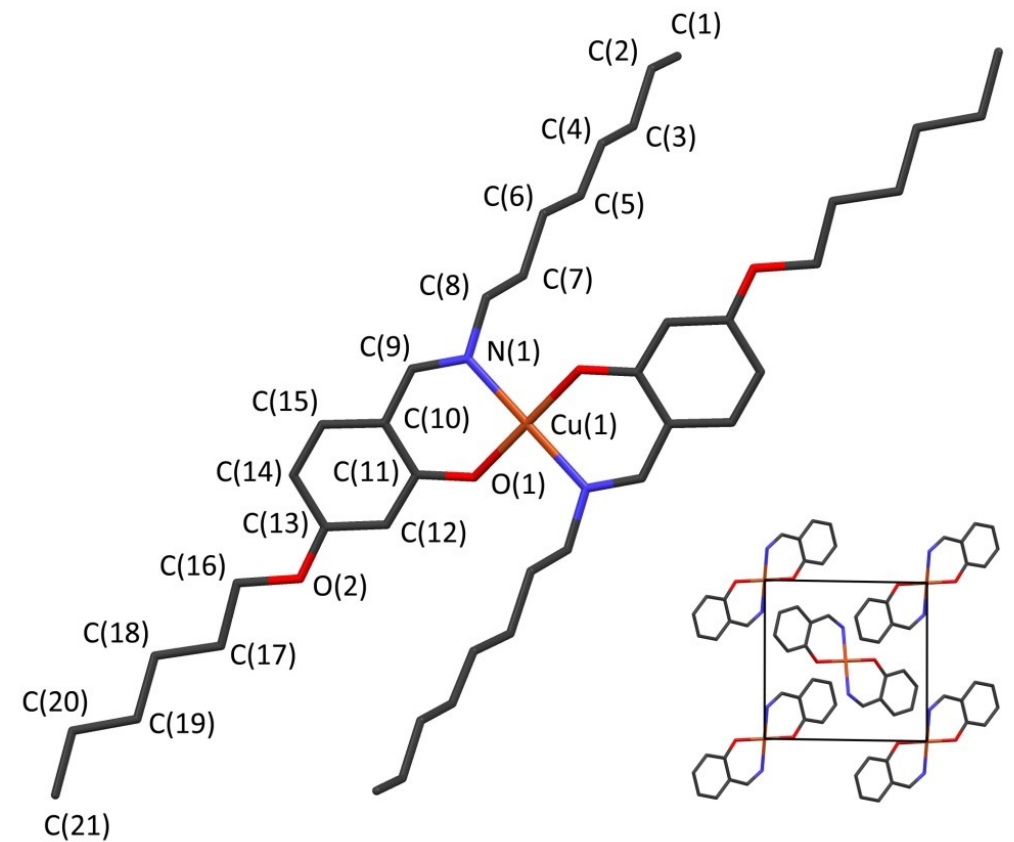

Figure 5.3.7: The structure of Cu1 $(8,6)$ as determined by single crystal X-ray crystallography viewed perpendicular to the core (main image) and the unit cell viewed down the c axis (inset). Note that as there is an inversion centre at $\mathrm{Cu}(1)$, the atoms in each ligand are equivalent and are only labelled once. The hydrogens in the structure have been omitted for clarity, as well as the tails in the insert.

Table 5.3.3: A selection of bond lengths, bond angles and dihedral angles in the structure of Cu1 $(8,6)$

\begin{tabular}{cccc}
\hline & Length / & & Angle / $^{\circ}$ \\
\hline $\mathrm{Cu}(1)-\mathrm{O}(1)$ & 1.897 & $\mathrm{~N}(1)-\mathrm{Cu}(\mathbf{1})-\mathrm{O}(1)$ & 91.19 \\
$\mathrm{Cu}(1)-\mathrm{N}(1)$ & 2.000 & $\mathrm{C}(9)-\mathrm{N}(\mathbf{1})-\mathrm{Cu}(1)$ & 122.19 \\
$\mathrm{C}(11)-\mathrm{O}(1)$ & 1.318 & $\mathrm{C}(11)-\mathrm{O}(\mathbf{1})-\mathrm{Cu}(1)$ & 128.02 \\
$\mathrm{C}(9)-\mathrm{N}(1)$ & 1.304 & $\mathrm{C}(10)-\mathrm{C}(11)-\mathrm{O}(\mathbf{1})-\mathrm{Cu}(1)$ & 18.75 \\
& & $\mathrm{C}(9)-\mathrm{N}(\mathbf{1})-\mathrm{C}(\mathbf{8})-\mathrm{C}(7)$ & 111.1 \\
\hline
\end{tabular}

Note that the atoms in bold are the central atom in the case of bond angles, or the bond along which the angle is measured in the case of dihedral angles.

Despite the complexes crystallising into two different space groups, they share some similarities. In particular, both compounds form highly segregated layer structures (Figure 5.3.8(a) and (b)). In the case of $\mathrm{Cu}(6,6)$, the layers are in the (100) plane 
whereas they are in the $(001)$ plane in $\mathrm{Cu}(8,6)$. The high degree of segregation of the layers is interesting in that one would expect favourable interactions between the tails that would lead to some interpenetration of the layers. A possible explanation comes from Date et $a l^{5}$ who claim that the presence of flexible chains in metallomesogens is a factor that inhibits crystallisation. This would indicate that the conformational flexibility of the alkoxy and $\mathrm{N}$-alkyl chains is the reason for the segregation. This conclusion is also borne out by the significant thermal motion of the chains observed in the structure, particularly the $\mathrm{N}$-alkyl chains (Figure 5.3.8(c) and (d)).
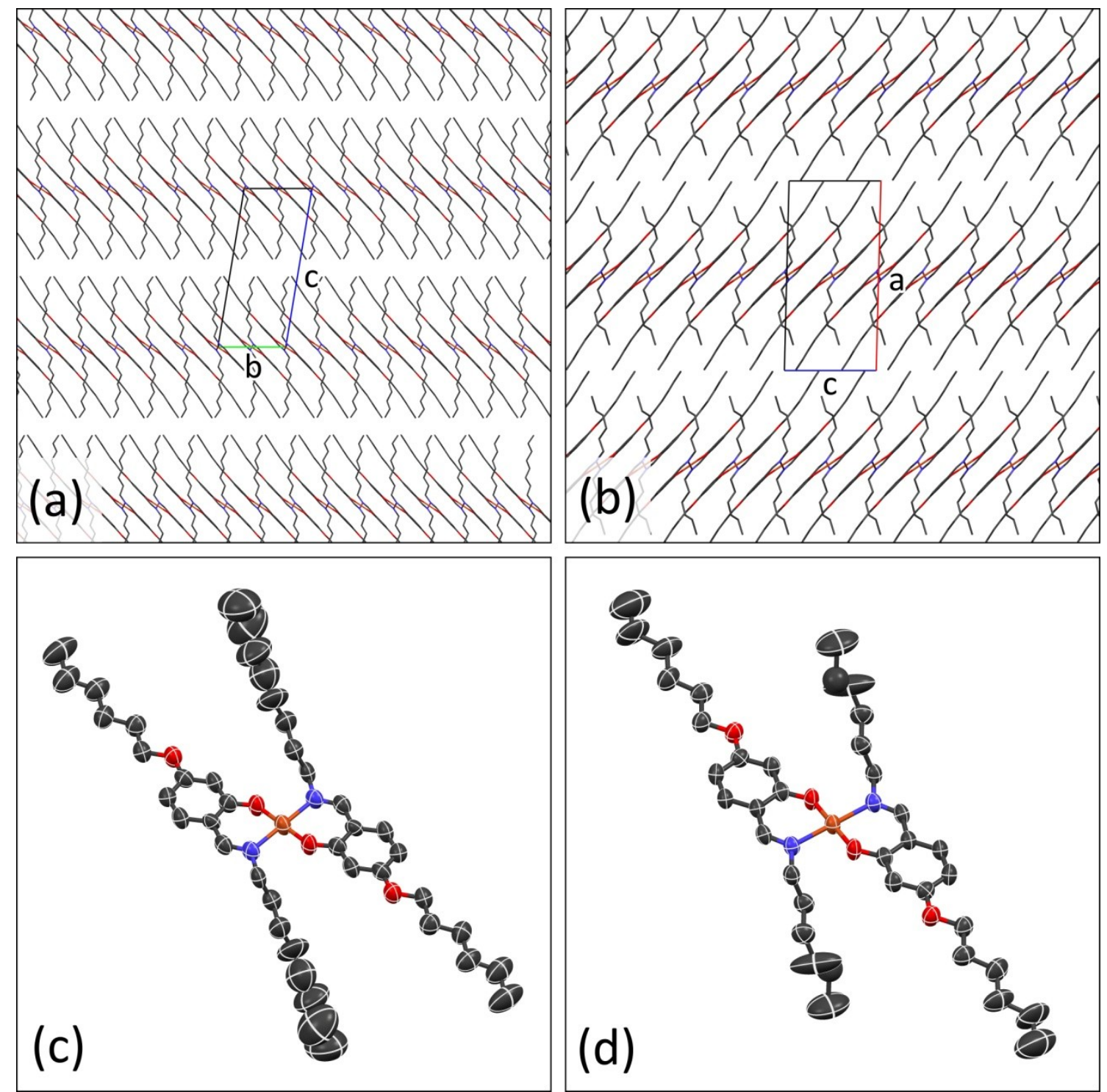

Figure 5.3.8: (a) Crystal packing of Cu1(8,6) in the (100) plane with the unit cell illustrated; (b) crystal packing of Cu1 $(6,6)$ in the (010) plane with the unit cell illustrated; (c) and (d) the 50\% probability thermal ellipsoid visualisation of the thermal motion of $\mathrm{Cu}(8,6)$ and $\mathrm{Cu}(6,6)$ respectively. Note that hydrogens and minority occupancy sites are omitted for clarity.

Single crystal diffraction was attempted for $\mathrm{Cr}_{2}$ (at $86^{\circ} \mathrm{C}$ for $\mathrm{Cu} 1(8,6)$ and $93^{\circ} \mathrm{C}$ for Cu1(6,6)). Unfortunately, upon transition to the $\mathrm{Cr}_{2}$ phase the crystals shattered, 
irrespective of how slowly the temperature was increased. This indicates that the $\mathrm{Cr}_{1^{-}}$$\mathrm{Cr}_{2}$ transition puts the crystal under considerable stress, potentially due to changes in the unit cell volume.

High temperature PXRD data were obtained for both complexes. As the temperature is increased in the $\mathrm{Cr}_{1}$ phase the unit cell undergoes anisotropic thermal expansion. In the case of $\mathrm{Cu}(8,6)$ the (001), (002) and (003) peaks show much less thermal expansion (shift to lower scattering vector) than the other peaks (Figure 5.3.9), indicating that thermal expansion of the unit cell mostly occurs in the plane of the layers and not perpendicular to the layers.

Table 5.3.4: Crystallographic data for the Cu1(8,6) and Cu1(6,6) single crystal X-ray diffraction experiments

\begin{tabular}{|c|c|c|}
\hline Complex & $\operatorname{Cu} 1(8,6)$ & $\operatorname{Cu} 1(6,6)$ \\
\hline Empirical Formula & $\mathrm{C}_{42} \mathrm{H}_{68} \mathrm{CuN}_{2} \mathrm{O}_{4}$ & $\mathrm{C}_{38} \mathrm{H}_{60} \mathrm{CuN}_{2} \mathrm{O}_{4}$ \\
\hline Molar mass / $\mathrm{g} \mathrm{mol}^{-1}$ & 728.52 & 672.42 \\
\hline Temperature / K & $293(2)$ & 292(2) \\
\hline Crystal System & Triclinic & Monoclinic \\
\hline Space Group; Z & $\mathrm{P} \overline{1} ; 2$ & $\mathrm{P} 2_{1} / \mathrm{c} ; 2$ \\
\hline $\mathrm{a} / \AA ̊ \AA ;$ / $^{\circ}$ & $9.5174(8) ; 99.371(7)$ & 20.4479(14); 90.00 \\
\hline b / $\AA ; \beta$ / $^{\circ}$ & $9.8343(9) ; 95.528(7)$ & 9.5148(3); 91.419(6) \\
\hline c / Å; V / & 23.4063(19); 90.072(6) & $9.9013(3) ; 90.00$ \\
\hline Volume / $\AA^{3}$ & 2151.15 & 1925.78 \\
\hline Density / $\mathrm{g} \mathrm{cm}^{-3}$ & 1.125 & 1.160 \\
\hline$\mu\left(\mathrm{Cu} \mathrm{K} \mathrm{K}_{\alpha}\right) / \mathrm{mm}^{-1}$ & 1.009 & 1.089 \\
\hline$F(000)$ & 790 & 726 \\
\hline$\theta$ range $/^{\circ}$ & $6.52 \rightarrow 44.49$ & $6.77 \rightarrow 72.13$ \\
\hline$h$ range & $-8 \rightarrow 8$ & $-25 \rightarrow 21$ \\
\hline $\mathrm{k}$ range & $-6 \rightarrow 8$ & $-11 \rightarrow 8$ \\
\hline I range & $-21 \rightarrow 21$ & $-12 \rightarrow 11$ \\
\hline $\begin{array}{l}\text { Reflections: total; } \\
\text { unique }\end{array}$ & $10523 ; 3329$ & $8484 ; 3546$ \\
\hline $\begin{array}{l}\text { Data; Restraints; } \\
\text { Parameters }\end{array}$ & $3329 ; 0 ; 449$ & $3546 ; 4 ; 206$ \\
\hline R; wR; Goodness of Fit & 7.65\%; 0.2017; 1.012 & 6.46\%; 0.1872; 1.094 \\
\hline
\end{tabular}

The same thermal expansion behaviour was observed in $\mathrm{Cu} 1(6,6)$ in the $\mathrm{Cr}_{1}$ crystalline phase (Figure 5.3.9). These data would suggest that the interactions between layers (principally dispersion forces) are stronger than the lateral interactions within layers.

Upon transition to the $\mathrm{Cr}_{2}$ phase the diffraction data for both complexes change significantly, indicating both complexes enter a new space group when entering the $\mathrm{Cr}_{2}$ crystal phase (Figure 5.3.10). The layered structure from the $\mathrm{Cr}_{1}$ phase is still present in 
the same spacing in $\mathrm{Cr}_{2}$ in both complexes, as indicated by the continued presence of the first, second and third order reflections due to the layers (Figure 5.3.10). The preservation of the layered structure shows that the structural change that occurs at the $\mathrm{Cr}_{1}-\mathrm{Cr}_{2}$ transition occurs within the layers and is therefore unlikely to be a significant change in orientation of the molecules.
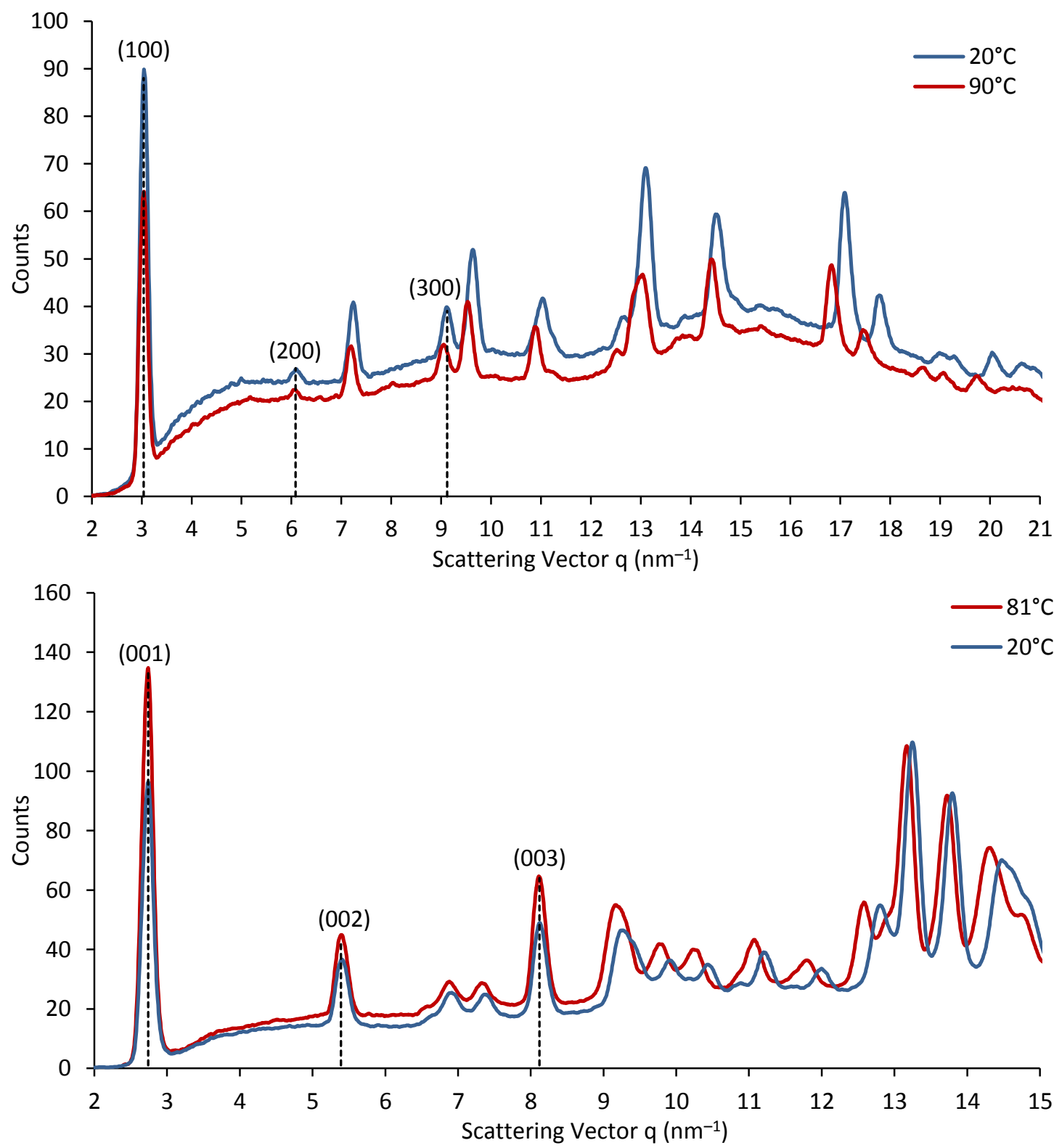

Figure 5.3.9: (top) Variable temperature PXRD data obtained from Cu1 $(6,6)$ in the $\mathrm{Cr}_{1} \mathrm{crystal}$ phase at $20^{\circ} \mathrm{C}$ and $81^{\circ} \mathrm{C}$ with the room temperature (100), (200) and (300) peak positions annotated; and (bottom) variable temperature PXRD data obtained from $\mathrm{Cu}(8,6)$ in the $\mathrm{Cr}_{1}$ crystal phase at $20^{\circ} \mathrm{C}$ and $81^{\circ} \mathrm{C}$ with the room temperature (001), (002) and (003) peak positions annotated.

As also observed in the DSC data, these changes are preserved when the complex is cooled below the transition temperature and the $\mathrm{Cr}_{1}$ phase is only restored after the sample has been allowed to relax for some time (see Section 5.3.1). Evidence of this was seen in samples of $\mathrm{Cu}(8,6)$ and $\mathrm{Cu} 1(6,6)$ cooled to $50^{\circ} \mathrm{C}$ from liquid and left to 
stand for 20 minutes. Features of both $\mathrm{Cr}_{1}$ and $\mathrm{Cr}_{2}$ are present in their respective PXRD data (Figure 5.3.11).
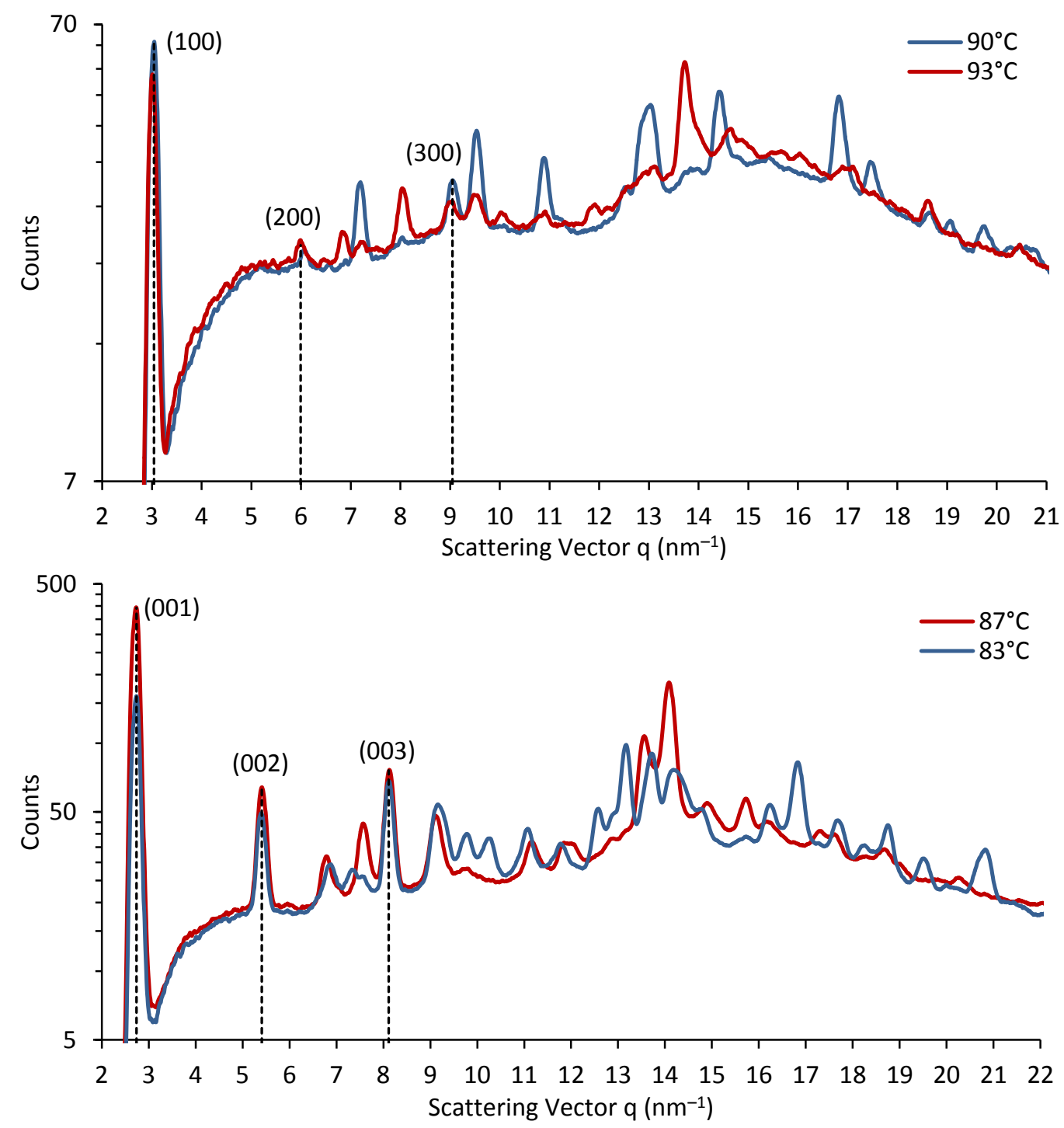

Figure 5.3.10: (top) variable temperature PXRD data obtained from $\mathrm{Cu} 1(6,6)$ at $90^{\circ} \mathrm{C}\left(\mathrm{Cr}_{1}\right)$ and $93^{\circ} \mathrm{C}\left(\mathrm{Cr}_{2}\right)$ with the $\mathrm{Cr}_{1}$

(100), (200) and (300) peak positions annotated; and (bottom) variable temperature PXRD data obtained from $\mathrm{Cu} 1(8,6)$ at $83^{\circ} \mathrm{C}\left(\mathrm{Cr}_{1}\right)$ and $87^{\circ} \mathrm{C}\left(\mathrm{Cr}_{2}\right)$ with the $\mathrm{Cr}_{1}(001),(002)$ and (003) peak positions annotated.

For both complexes the $\mathrm{Cr}_{1}-\mathrm{Cr}_{2}$ transition results in a decrease in the unit cell volume, causing the crystal to shatter. This change in volume occurs within the plane of the layers. From a molecular perspective, the most likely causes of this are structural changes that involve the conformation of the $\mathrm{N}$-alkyl chains, such as rotation to lie in the plane of the core. Such rotation would result in a more planar molecule allowing a more dense packing, resulting in a smaller unit cell. This hypothesis is supported by the large amount of thermal motion that the $\mathrm{N}$-alkyl chains have within the crystal structure, indicating their conformational sensitivity to changes in temperature. Such a change in chain conformation is unlikely to alter the layer spacing as that is primarily 
determined by the alkoxy chains, and the lateral interactions between the molecules are much weaker than the inter-layer interactions (as shown by the anisotropic thermal expansion). This hypothesis may also help explain some features of the phase relaxation kinetics, which are discussed further in Section 5.3.3.
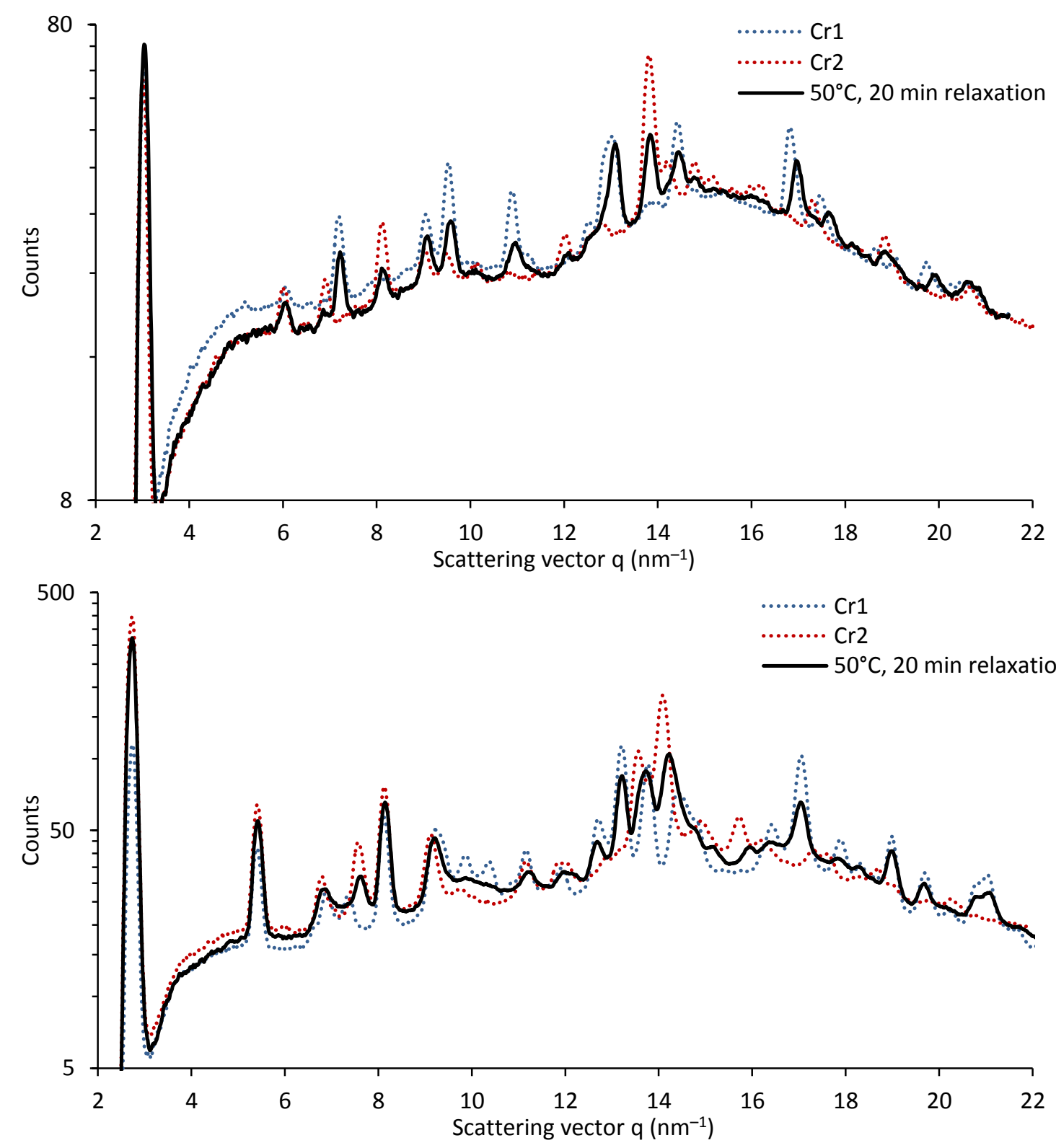

Figure 5.3.11: PXRD data obtained from samples of (top) Cu1(6,6) and (bottom) Cu1(8,6) cooled from liquid to $50^{\circ} \mathrm{C}$ and left to stand at $50^{\circ} \mathrm{C}$ for 20 minutes. The samples have peaks from both their respective $\mathrm{Cr}_{1}$ and $\mathrm{Cr}_{2}$ phases, which are shown in blue and red respectively for reference.

\subsubsection{Kinetics of Phase Relaxation}

As mentioned in Section 5.3.1, the Cu1 complexes crystallise from the liquid state into the $\mathrm{Cr}_{2}$ phase, and remain in that phase when cooled below the $\mathrm{Cr}_{1}-\mathrm{Cr}_{2}$ transition temperature. Relaxation to the $\mathrm{Cr}_{1}$ phase only occurs when the sample has been left to stand below the transition temperature, or when a very slow cooling rate is employed 
(ca. $-5^{\circ} \mathrm{Chr}^{-1}$ ). This phase relaxation behaviour was monitored using DSC (Figure

\subsubsection{2).}

A typical experiment consisted of heating the sample from room temperature into the $\mathrm{Cr}_{2}$ phase. The heat of the transition $\left(Q_{\max }\right)$ was estimated from the data. The sample was then further heated into the fluid phase, cooled to the desired relaxation temperature $(T)$, and rested at that temperature for a known amount of time $(\Delta t)$. Following this, the sample was heated into the $\mathrm{Cr}_{2}$ phase, and the heat of transition $\left(\mathrm{Q}_{\Delta t}\right)$ determined. The mole fraction of sample that had relaxed to $\mathrm{Cr}_{1}$ during the rest time is obtained from the ratio of $Q_{\Delta t}$ to $Q_{\max }$. This process was repeated for a range of rest times and temperatures.

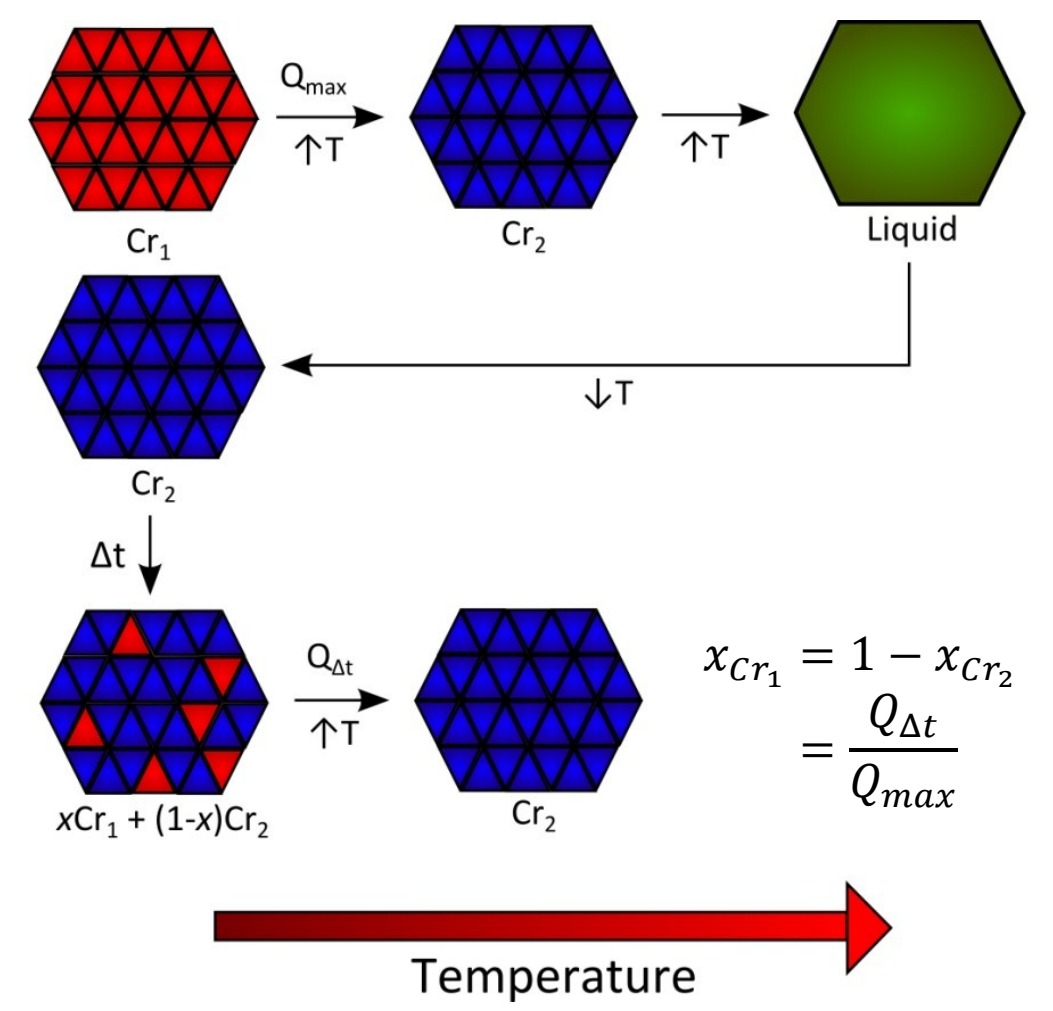

Figure 5.3.12: The DSC procedure followed to determine the mole fraction of $C r_{1}\left(x_{C r_{1}}\right)$ in a sample after it has been left to stand at a known temperature for an amount of time ( $\Delta t)$ from the heat (Q) of the $\mathrm{Cr}_{1}-\mathrm{Cr}_{2}$ transition.

The typical relaxation behaviour consists of an exponential decay as a function of $\mathrm{Cr}_{2}$ mole fraction following an induction period (Figure 5.3.13). The exponential decay is consistent with first order kinetics:

$$
[\mathrm{B}]=[\mathrm{B}]_{0} e^{-k t}
$$

where $[B]$ is the mol fraction of the reactant, $[B]_{0}$ is the initial mole fraction of the reactant, $t$ is the reaction time and $k$ is the reaction rate. In this system $[B]_{0}$ is 1 as at 
zero relaxation time the entire sample is in the $\mathrm{Cr}_{2}$ phase. The presence of an induction period is not accounted for in this model; most likely it arises from thermal diffusion.

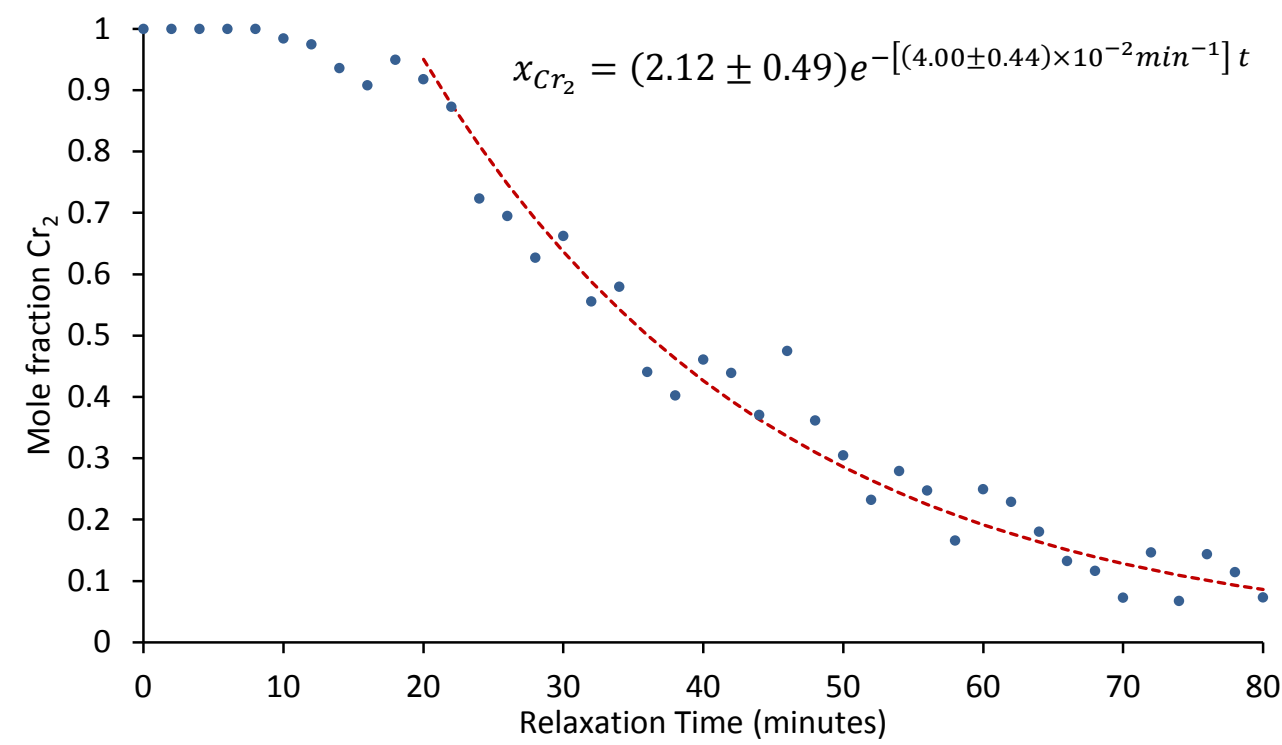

Figure 5.3.13: Phase relaxation data for $\mathrm{Cu} 1(8,6) ; T=43.7^{\circ} \mathrm{C}$. The data were fitted with a decaying exponential (red) by the sum of least squares method. Uncertainties represent a $95 \%$ confidence interval in the fit.

Relaxation data were collected at a range of temperatures in order to determine the activation energy of the relaxation via the Arrhenius equation:

$$
k=A e^{-E_{a} / R T}
$$

where $E_{a}$ is the activation energy, $R$ is the universal gas constant, $T$ is the temperature and $A$ is the pre-exponential constant. The fitting of this equation yields a negative apparent activation energy $\left(-21.3 \pm 5.0 \mathrm{~kJ} \mathrm{~mol}^{-1}\right.$ with $95 \%$ confidence for Cu1(8,6), Figure 5.3.14) indicating that the relaxation actually occurs with at least a competing pre-equilibrium. A negative apparent activation barrier was also estimated for $\mathrm{Cu}(6,6)$ $\left(-106 \pm 16 \mathrm{~kJ} \mathrm{~mol}^{-1}\right.$ determined with 95\% confidence, Figure 5.3.15).

These data further support the hypothesis that the $\mathrm{N}$-alkyl chains are responsible for the $\mathrm{Cr}_{1}-\mathrm{Cr}_{2}$ crystal phase transition, as increasing the length of the chains by only two carbons has the effect of decreasing the magnitude of the negative activation barrier by five fold. This shows that the kinetics of the pre-equilibrium are highly sensitive to the $\mathrm{N}$-alkyl chain length, which means that the $\mathrm{N}$-alkyl chains must be involved in the pre-equilibrium in some capacity. 


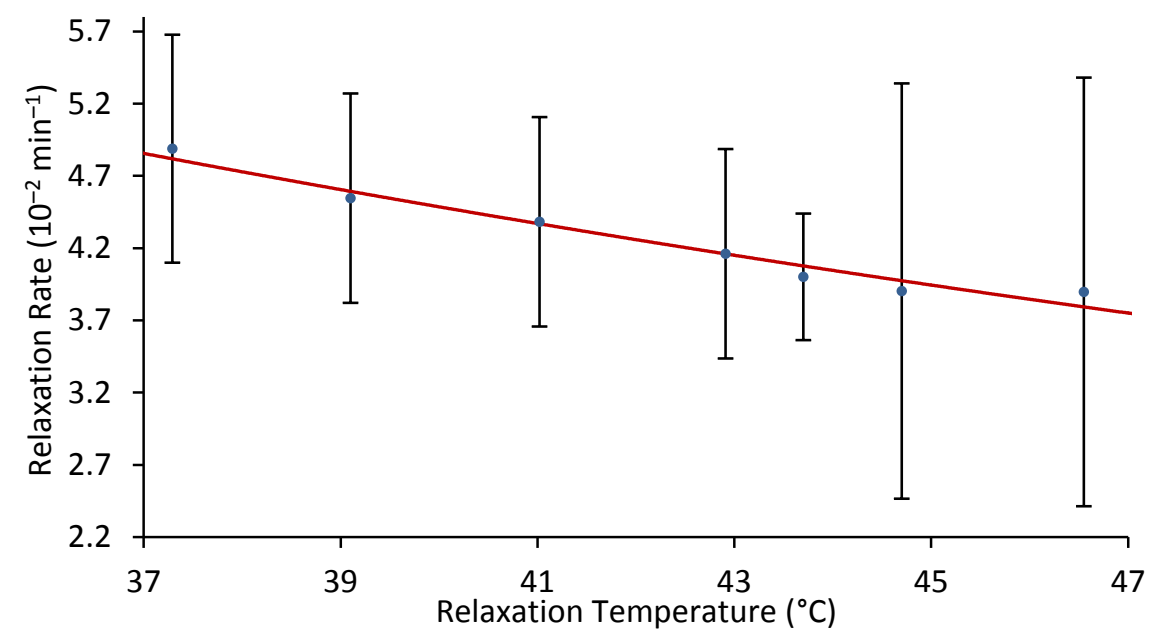

Figure 5.3.14: Temperature dependence of the phase relaxation data measured from Cu1 $(8,6)$. The fit of the Arrhenius equation is shown in red.

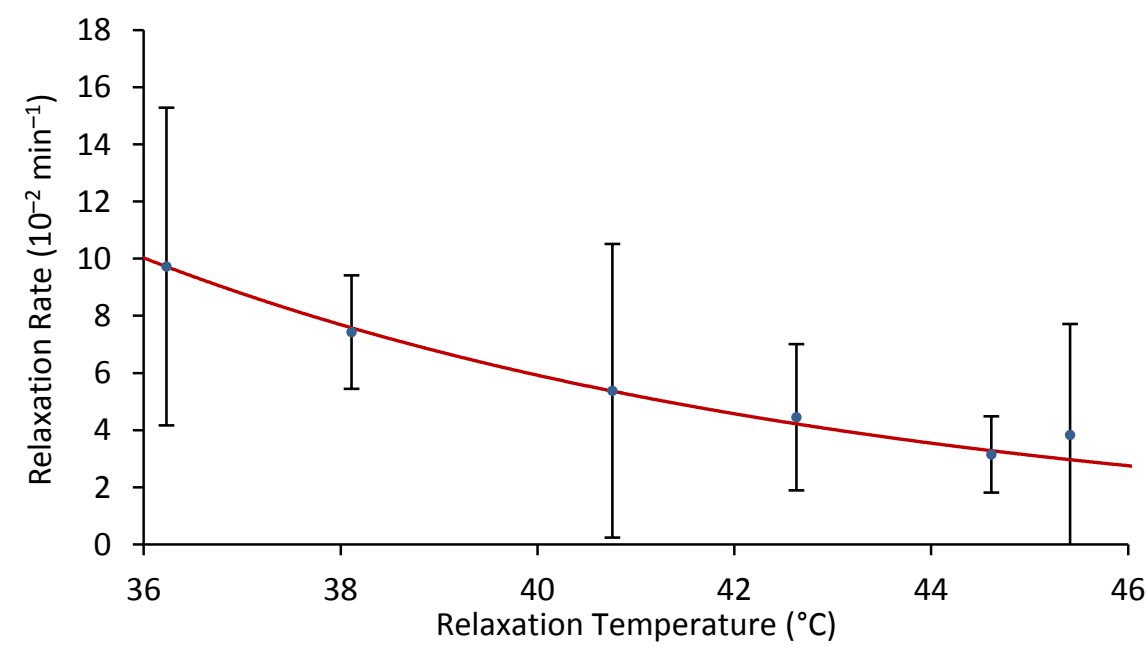

Figure 5.3.15: Temperature dependence of the phase relaxation data measured from Cu1(6,6). The fit of the Arrhenius equation is shown in red.

\subsection{Binuclear N-alkyl Salicylaldimine Complexes $\mathrm{Cu} 2$}

Through extension of both the anisotropy and flexibility of the complexes via formation of the bimetallic species reported by Paschke et al., ${ }^{50}$ induction of mesomorphism was expected. When $\mathrm{Cu2}(10,6)$ is heated from the crystalline phase the sample undergoes three transitions: at $63.6^{\circ} \mathrm{C}\left(-10.1 \pm 0.9 \mathrm{~kJ} \mathrm{~mol}^{-1}\right)$, at $126.2^{\circ} \mathrm{C}$ $\left(-50.2 \pm 0.9 \mathrm{~kJ} \mathrm{~mol}^{-1}\right)$ and at $131.2^{\circ} \mathrm{C}\left(-3.1 \pm 0.9 \mathrm{~kJ} \mathrm{~mol}^{-1}\right)$ (Figure 5.4.1). Only two transitions appear on cooling, one at $64.1^{\circ} \mathrm{C}\left(13.5 \pm 0.9 \mathrm{~kJ} \mathrm{~mol}^{-1}\right)$ and $51.8^{\circ} \mathrm{C}$ $\left(5.4 \pm 0.9 \mathrm{~kJ} \mathrm{~mol}^{-1}\right)$. Consideration of the enthalpies of the transitions on heating and on cooling indicates that the sample has not returned its initial crystalline state.

This is confirmed via application of a second cycle in which the cooling behaviour is identical, but the sample now only has a single broad transition on heating at $88.9^{\circ} \mathrm{C}$ 
$\left(-18.2 \pm 0.9 \mathrm{~kJ} \mathrm{~mol}^{-1}\right)$. The breadth of the transition on heating indicates that there are now multiple transitions occurring at the melting point and/or the purity of the sample has substantially decreased. This behaviour was also seen in Cu2(8,8), with transitions at almost exactly the same temperatures and similar enthalpies. It is most likely that the complexes are not stable and that decomposition occurs at $126^{\circ} \mathrm{C}$.

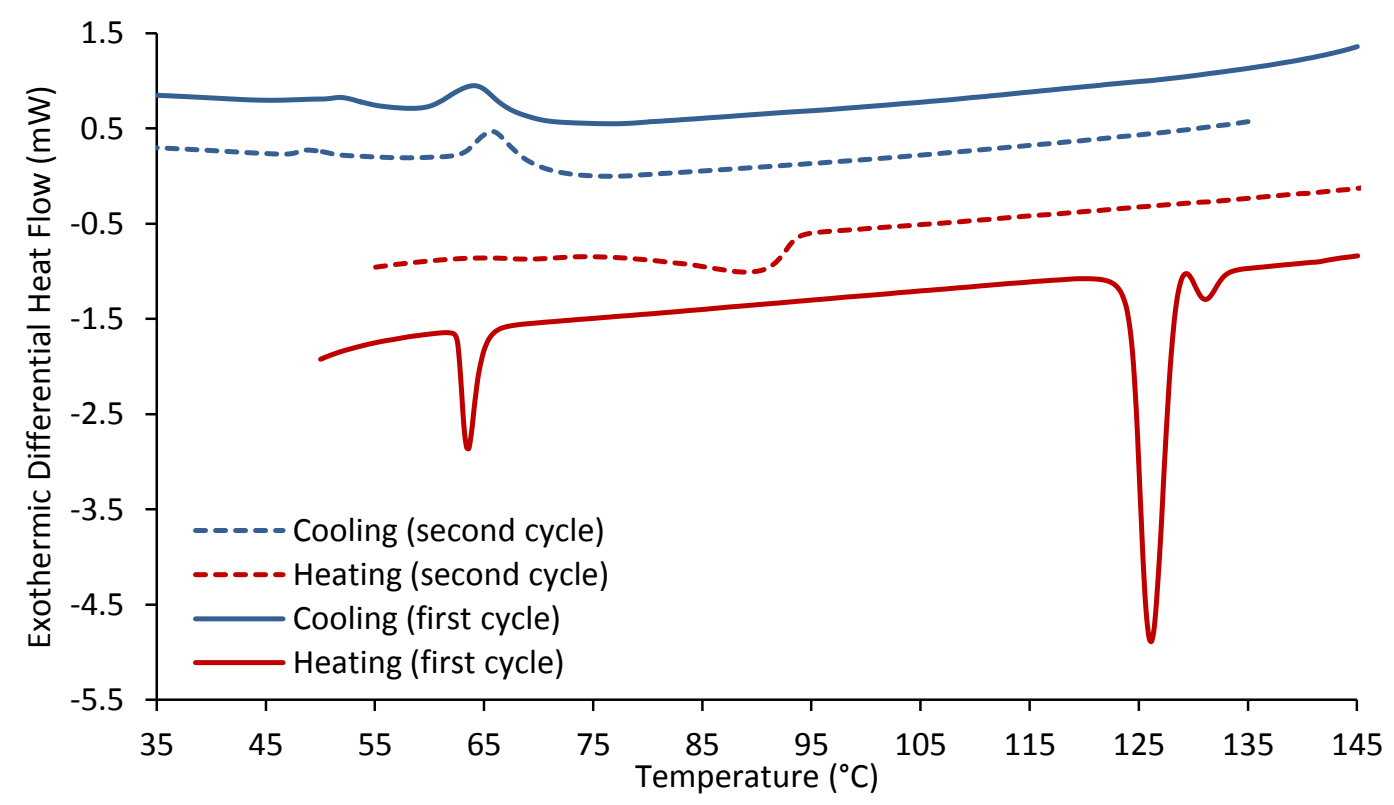

Figure 5.4.1: DSC data obtained on the first and second cycles of Cu2(10,6).

Decomposition is also confirmed from POM observations (Figure 5.4.2). The sample remains in a birefringent crystalline phase until $122^{\circ} \mathrm{C}$ at which point the sample becomes isotropic. Upon cooling the sample appears to crystallise into two different crystalline textures around $60^{\circ} \mathrm{C}$ (Figure 5.4 .2 (left)). When heated again, one texture melts before the other (Figure 5.4.2(right)), and the sample becomes isotropic again at $90^{\circ} \mathrm{C}$.
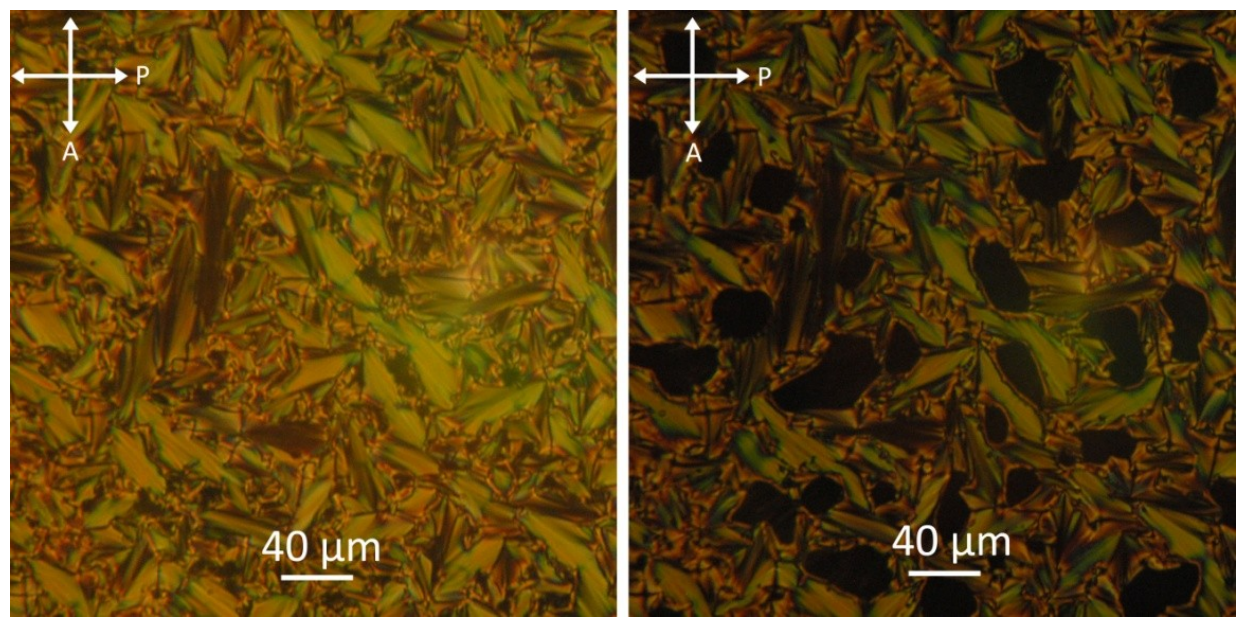

Figure 5.4.2: POM images of Cu2 $(8,8)$ captured at (left) $50^{\circ} \mathrm{C}$ and (right) $83^{\circ} \mathrm{C}$. The polariser (P) and analyser (A) orientations are shown. 
Paschke et al. noted that the "weaker $\mathrm{Cu}-\mathrm{Cl}$ bond causes lower chemical stability" and that it is "fairly surprising that the binuclear $N$-alkyl-substituted complexes... exhibit monotropic and enantiotropic liquid crystalline behaviour". ${ }^{50}$ Hence the instability observed is most likely due to the lability of the $\mathrm{Cu}-\mathrm{Cl}$ bond. This problem might be remedied by using a less labile ligand, or longer alkyl tails as was done by Paschke et al., ${ }^{50}$ however this goes beyond the scope of this research.

\subsection{N-(4-butylphenyl) Salicylaldimine Complex and Ligand}

\subsubsection{Structure and Phase Behaviour of $L 3(4,6)$}

As mentioned in Section 4.2 the $L 3(4,6)$ ligand (Figure 5.5.1) is crystalline at room temperature, and melts to a cloudy fluid slightly above room temperature, finally clarifying at higher temperatures, reminiscent of Reinitzer's original observations of cholesterol benzoate. ${ }^{6,8}$ This observation is, however, hard to reconcile with the DSC data (Figure 5.5.2), which show only a single melting peak at $42.40^{\circ} \mathrm{C}$ on heating $\left(13.9 \pm 0.4 \mathrm{~kJ} \mathrm{~mol}^{-1}\right)$ and no corresponding peak on cooling, rather there is a small peak $\left(250 \pm 350 \mathrm{~J} \mathrm{~mol}^{-1}\right)$ at $15.08^{\circ} \mathrm{C}$. Upon reheating, a peak at $17.43^{\circ} \mathrm{C}\left(550 \pm 350 \mathrm{~J} \mathrm{~mol}^{-1}\right)$ corresponding to the small cooling peak is present, but the large melting peak is no longer evident, indicating that the sample does not re-form the crystalline phase on cooling.

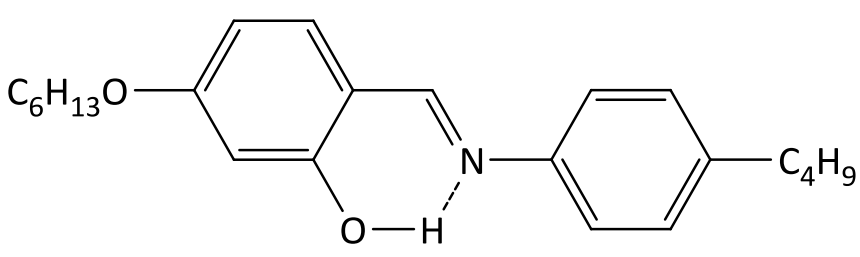

Figure 5.5.1: The structure of $L 3(4,6)$.

POM provides a clearer picture of what changes are occurring in the sample. When heating a crystalline sample past $42^{\circ} \mathrm{C}$ the sample enters a fluid, highly birefringent phase (Figure 5.5.3(a)). The texture of this phase is consistent with the Schlieren texture of a nematic mesophase. ${ }^{4}$ A large number of defects (Figure 5.5.3(b), (c) and (d)) develop as the temperature is raised, which begin to disappear at $60^{\circ} \mathrm{C}$ and the sample appears isotropic at $65^{\circ} \mathrm{C}$. When cooled below $60^{\circ} \mathrm{C}$ the nematic mesophase is observed again until $30^{\circ} \mathrm{C}$ when it disappears again, replaced by a viscous phase with little birefringence. This means that the sample is enantiotropic. Sub-ambient temperature observations were not able to be carried out. 


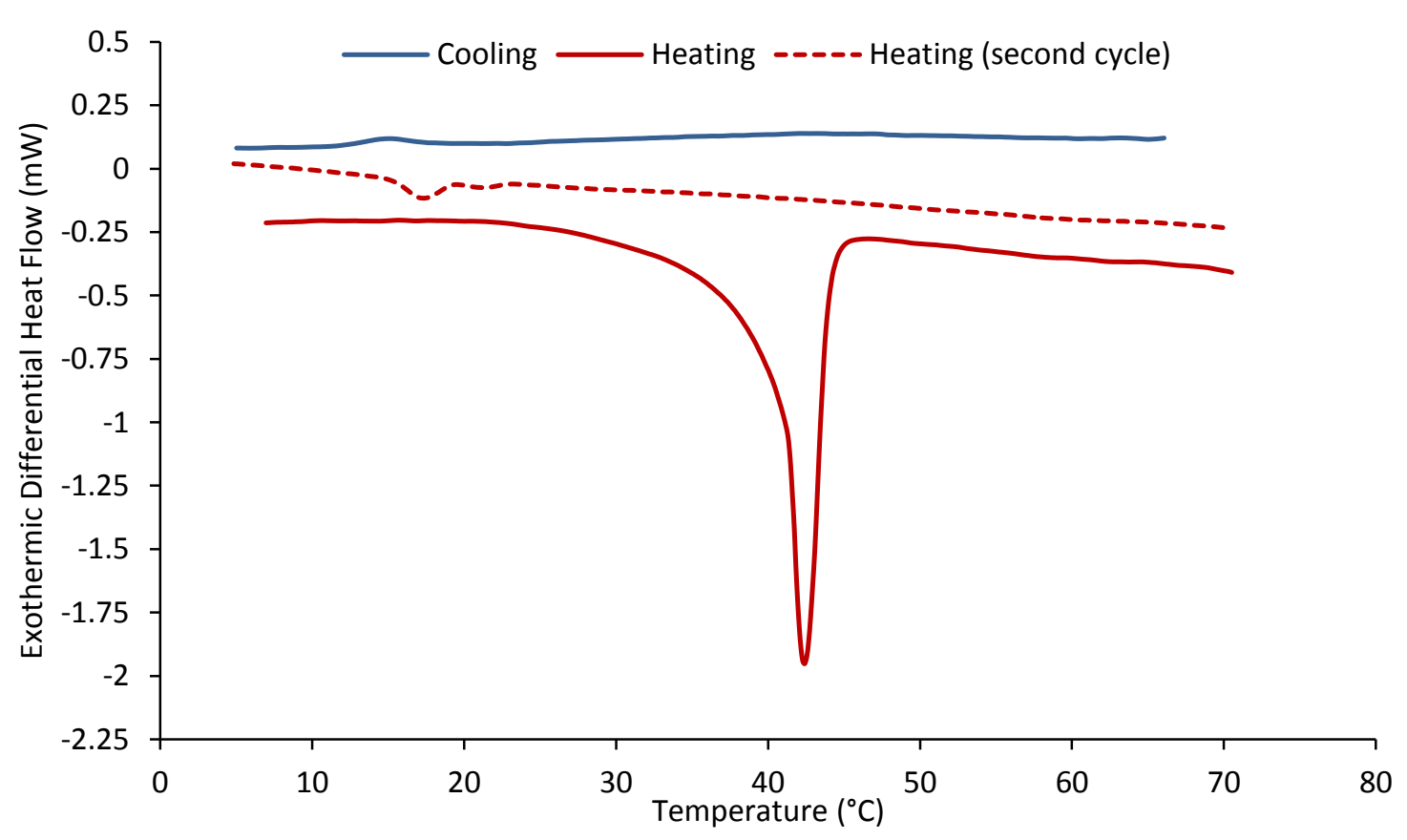

Figure 5.5.2: DSC data measured from a sample of $L 3(4,6)$. Cycle one: $\pm 7.0^{\circ} \mathrm{C} \mathrm{min}^{-1}$, cycle two: $5.0^{\circ} \mathrm{C} \mathrm{min}^{-1}$.
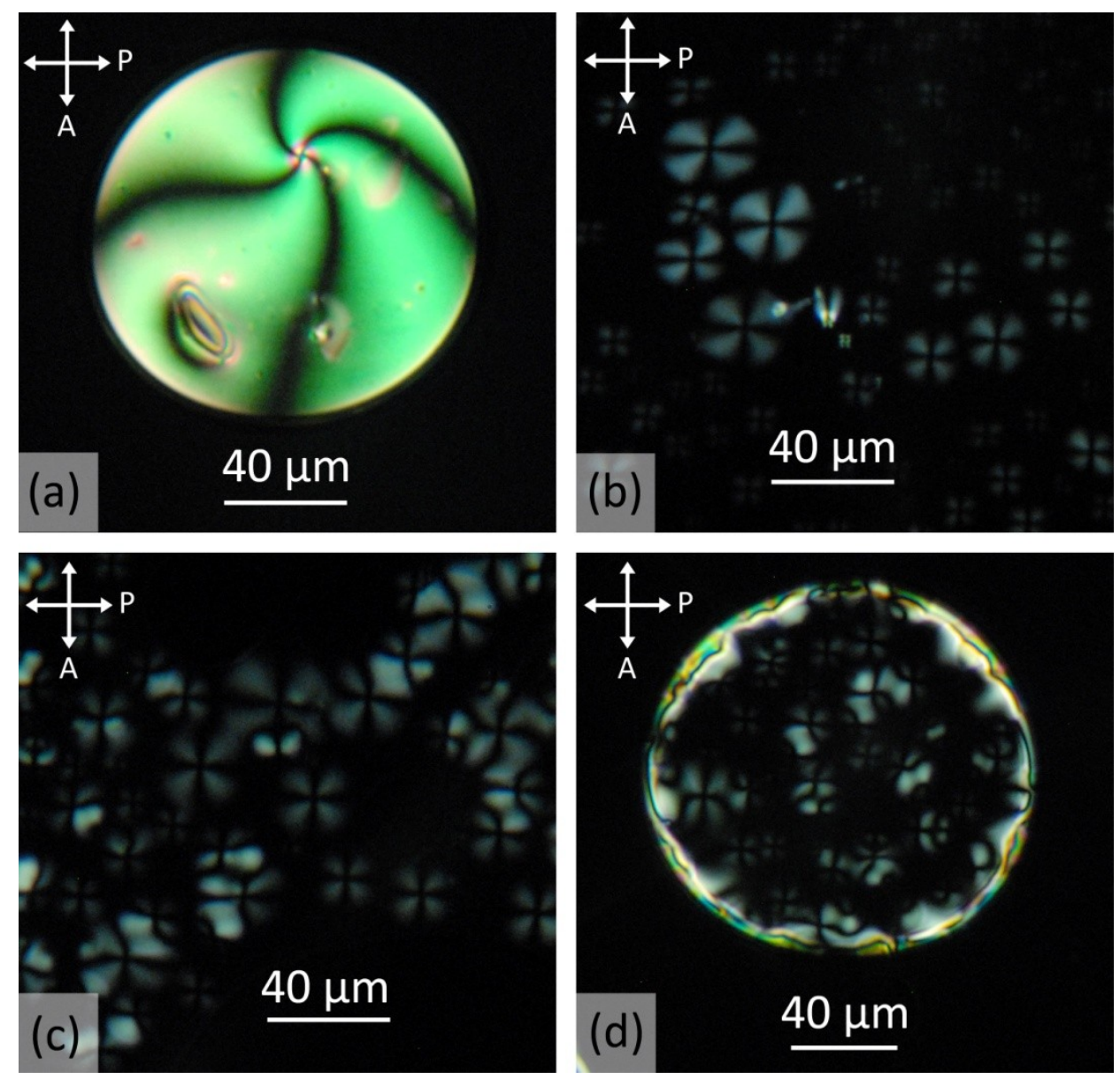

Figure 5.5.3: Examples of the optical textures formed by $L 3(4,6)$ in the nematic mesophase. POM images were captured at: (a) $44.0^{\circ} \mathrm{C}$; (b) $57.7^{\circ} \mathrm{C}$; (c) $57.7^{\circ} \mathrm{C}$; and (d) $55.0^{\circ} \mathrm{C}$. The polariser $(P)$ and analyser $(A)$ orientations are shown.

In Figure 5.5.3 a number of disclinations are evident, appearing as the "Maltese cross" shape (Figure 5.5.3(b)) or the thread-like texture in Figure 5.5.3(a) or a combination of 
the two. This distinctive cross shape forms due to the orientation of the mesogens around the disclination. In the middle of the dark brushes the director field is parallel or perpendicular to the polariser and analyser, and in the middle of the light brushes they are at $45^{\circ}$ (see Appendix A). It follows that in a cross-shaped defect, the director field reverses direction around the disclination. This is known as a \pm 1 strength disclination. In general, the disclination strength of a defect can be found from the angle between the dark brushes of the texture:

$$
|s|=\frac{180}{2 \theta}
$$

where $s$ is the strength of the disclination and $\theta$ is the angle between dark brushes in degrees. ${ }^{4}$ Whether the strength is positive or negative relates to the direction that the texture rotates when the polariser and analyser are rotated together. If the defect rotates in the same direction as the polariser and analyser then it is positive, and if it rotates in the opposite direction it is negative. The speed at which it rotates is also related to the disclination strength:

$$
\omega_{T}=\frac{\omega_{P}}{S}
$$

where $\omega_{T}$ is the rotation rate of the texture and $\omega_{P}$ is the rotation rate of the polarizing filters. ${ }^{4}$ POM therefore allows one to completely characterise disclinations in a mesophase in terms of both their strength and sign. ${ }^{36}$ In the nematic mesophase of $L 3(4,6)$ the vast majority of defects observed were +1 , with some $+1 / 2$ defects observed near interfaces (Figure 5.5.3(d)).

Reconciliation of the DSC and POM data requires consideration of the interactions between mesogens in a nematic phase and the interactions in an isotropic liquid. $A$ nematic phase is essentially a 1D aligned liquid. Energetically there is very little difference between the nematic and liquid phases. As such the heat of a nematicisotropic transition can be very low, ${ }^{54}$ typically less than $1 \mathrm{~kJ} \mathrm{~mol}^{-1}$ and if the transition is broad it can be very difficult to observe.

In order to understand how the structure changes at the transitions observed above, small and small/wide angle X-ray scattering (SAXS and WSAXS respectively) experiments were carried out at a range of temperatures. At small angles, the 
crystalline phase has a single sharp peak, which corresponds to order on the longitudinal axis of the mesogen (Figure 5.5.4(a)). When the sample enters the nematic mesophase, this peak disappears, replaced by a smaller, broader peak, indicating a loss of translational order along the longitudinal axis of the mesogen (Figure 5.5.4(b)). This peak broadens and declines in intensity as the temperature is increased, until the sample enters the isotropic liquid (Figure 5.5.4(c)), showing that the mesophase becomes less ordered as the temperature is raised.
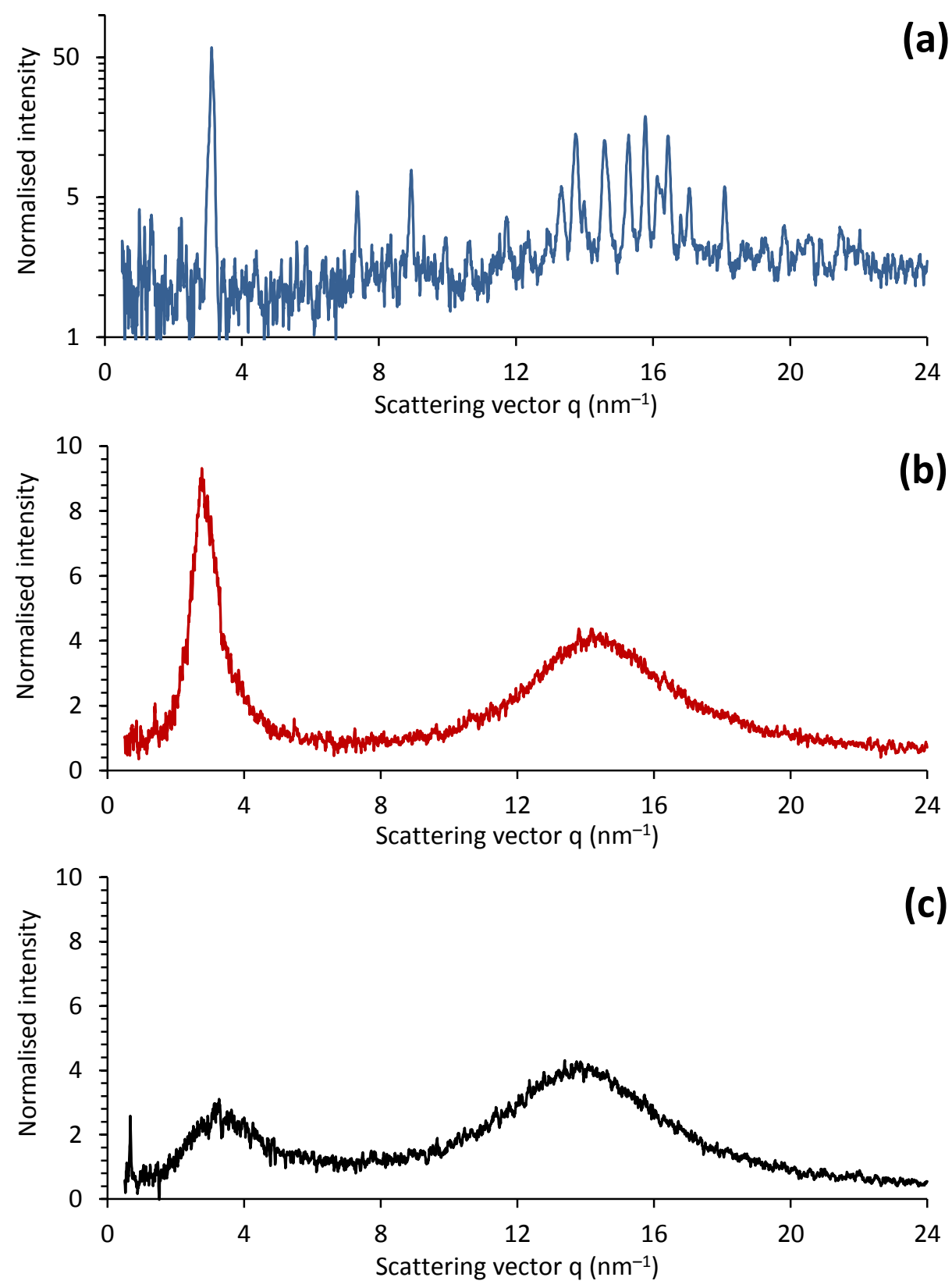

Figure 5.5.4: WSAXS data measured from $L 3(4,6)$ in the: (a) crystalline; (b) nematic; and (c) isotropic liquid phases. The data were measured on a point collimated beamline.

The behaviour at wide angles is less varied by comparison. In the crystalline phase there is a number of sharp peaks at wide angles resulting from reflections off the 
crystal lattice. The nematic and isotropic liquid phases both have a single diffuse peak at $14 \mathrm{~nm}^{-1}$ which does not vary significantly between the phases, aside from a small amount of thermal expansion to smaller scattering vector. This peak is due to order in the lateral direction to the mesogen structure. This will be similar in the nematic and isotropic liquid phases.

Using SAXS and WSAXS it is possible to examine the phase behaviour of $L 3(4,6)$ as it is cooled from the isotropic liquid phase. The DSC data indicate that the sample does not re-form the crystalline phase upon cooling, but undergoes a low energy phase change at $15.1^{\circ} \mathrm{C}$. When the sample is cooled below $40^{\circ} \mathrm{C}$ the broad peak at small angles significantly expands to lower scattering vector and increases in intensity beyond what was observed in the nematic mesophase (Figure 5.5.5). In addition the peak narrows significantly below $40^{\circ} \mathrm{C}$, as evidenced by the full width half-maximum (FWHM) data (Figure 5.5.5).
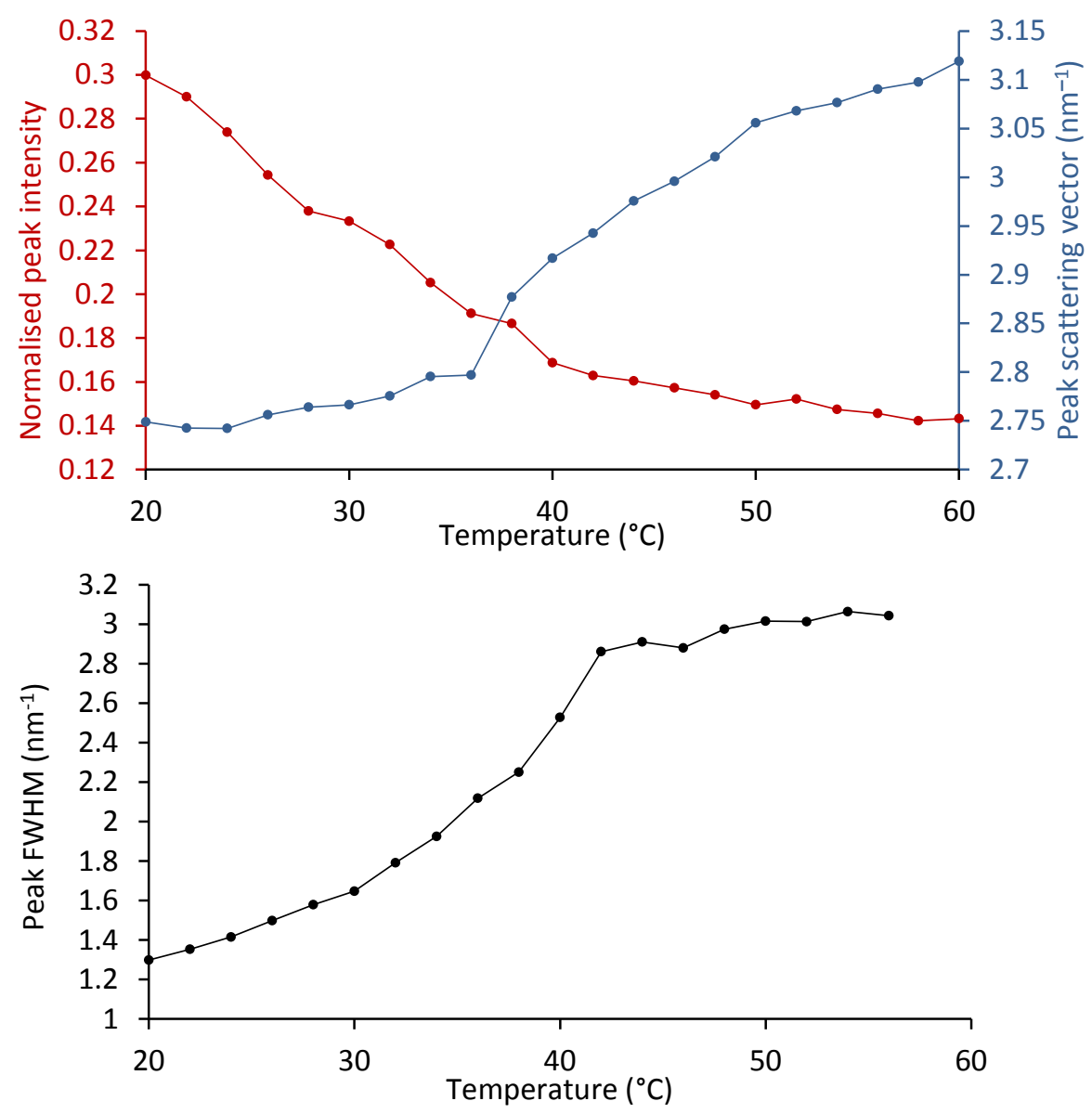

Figure 5.5.5: (top) Peak intensity and peak scattering vector; (bottom) peak FWHM dependence on temperature in the SAXS measurements of $L 3(4,6)$ as the temperature is lowered from the nematic mesophase.

The SAXS data indicate a structural change in the sample that is not evident in the DSC data. This indicates that the change is not a formal phase change, but supercooling of 
the nematic mesophase. This phenomenon is fairly common in nematic liquid crystals as, unlike isotropic liquids, nematic mesophases supercool at even slow cooling rates and are stable in the supercooled state, making this state difficult to avoid. ${ }^{64}$ Crystallisation of $L 3(4,6)$ can be induced by addition of nucleation sites, or cooling to very low temperatures (below $-10^{\circ} \mathrm{C}$ ).

When cooled further, below $15.1^{\circ} \mathrm{C}$, a strong peak at small angles appears, however from the wide angle region the sample remains fluid (Figure 5.5.6). This indicates that rather than fully crystallising, the sample has formed a partially amorphous solid (known as a plastic crystal ${ }^{4}$ ), which would be consistent with the low heat of the transition.

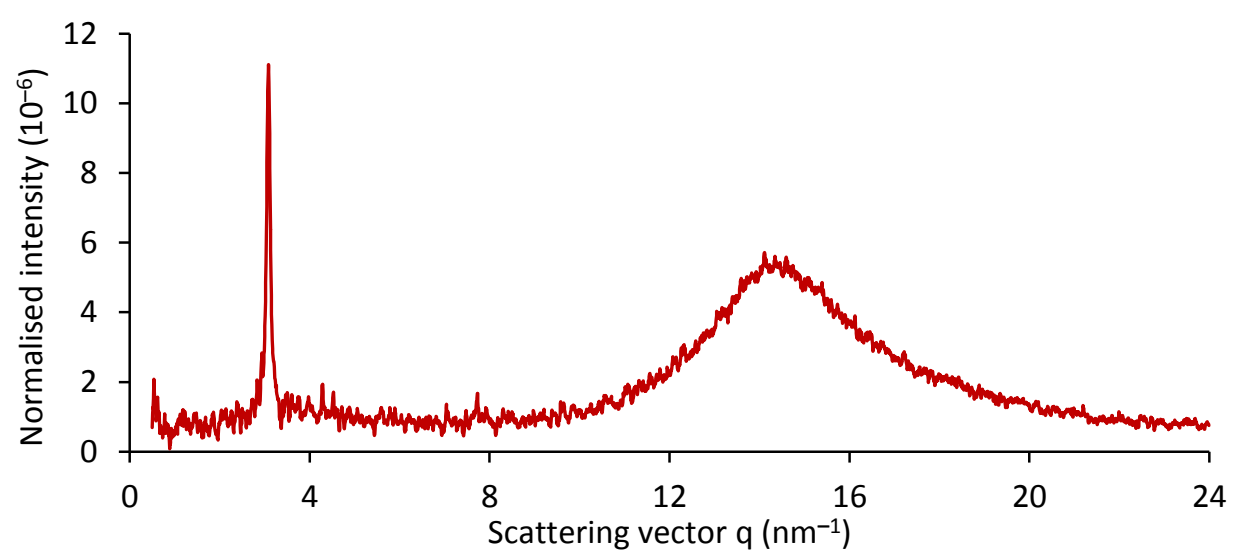

Figure 5.5.6: WSAXS data measured from $L 3(4,6)$ at $-10^{\circ} \mathrm{C}$ after cooling from the nematic mesophase. The data were measured on a point collimated beamline.

When heated from the plastic crystal phase, $L 3(4,6)$ melts to the supercooled nematic mesophase between $20^{\circ} \mathrm{C}$ and $25^{\circ} \mathrm{C}$. This is followed by a transition to the nematic mesophase between $40^{\circ} \mathrm{C}$ and $45^{\circ} \mathrm{C}$ and the clearing point at $60^{\circ} \mathrm{C}$. It is possible to determine the temperatures at which these transitions take place by examining the changes in the peak maximum (intensity and scattering vector) and the FWHM of the peak (Figure 5.5.7). The trends in peak scattering vector relate to how the average distance between mesogens changes with temperature, while the peak intensity and FWHM relate to how ordered the system is. Within each phase there is a trend towards less order (greater FWHM and lower intensity) and closer spacing (higher scattering vector) with increasing temperature, however there is discontinuity in the slope at each phase boundary. This should be expected as the interactions between mesogens in each phase are different, so the thermal response of the phases should also be different. 

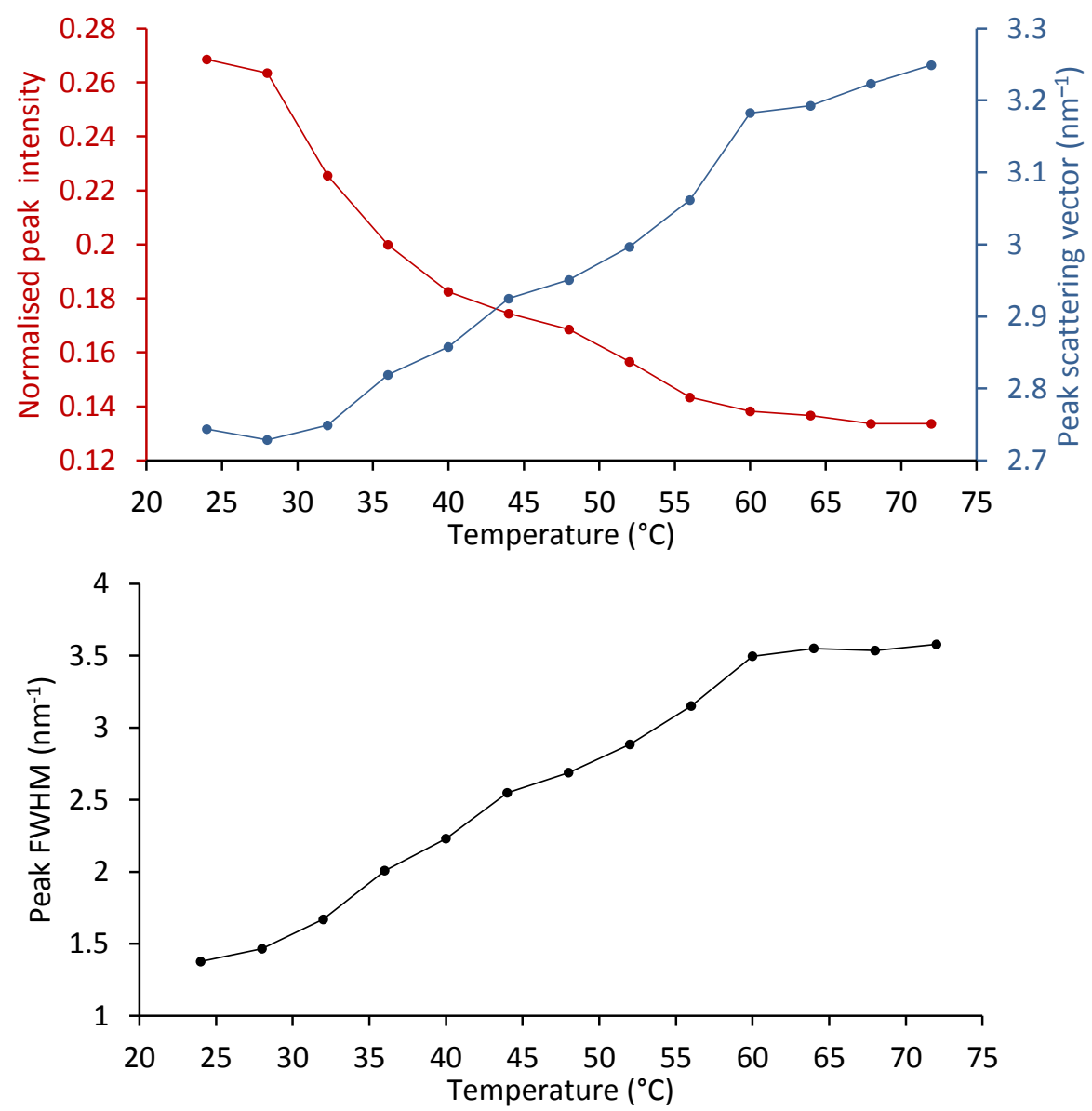

Figure 5.5.7: (top) Peak intensity and peak scattering vector; (bottom) peak FWHM dependence on temperature in the SAXS measurements of $L 3(4,6)$ as the temperature is raised from the supercooled nematic mesophase.

The trend to closer packing along the longitudinal axis with increasing temperature may seem unusual, as it appears to contradict the usual thermal expansion that most materials exhibit. However this peak is due to spacing on the longitudinal axis only, not laterally to the mesogen in which thermal expansion is observed (Figure 5.5.4(b) and (c)). The nematic phase exists on a continuum between highly ordered and highly disordered states. ${ }^{4}$ As the phase moves from one to the other the mesogens can pack together closer on the longitudinal axis as they (on average) orientate less and less tail to tail. This would lead to overall expansion, however, as the packing becomes less and less efficient.

In summary, the phase behaviour of $L 3(4,6)$ is now well understood. The compound melts to the nematic mesophase at $42^{\circ} \mathrm{C}$, which persists up to the clearing point at $60^{\circ} \mathrm{C}$. When cooled from the isotropic liquid it forms a nematic mesophase at $60^{\circ} \mathrm{C}$. This supercools when the temperature is lowered below $40^{\circ} \mathrm{C}$ and eventually forms a partially amorphous solid at $15.0^{\circ} \mathrm{C}$. When reheated, the compound enters the supercooled nematic phase around $20^{\circ} \mathrm{C}$ and then the nematic phase between $40^{\circ} \mathrm{C}$ 
and $45^{\circ} \mathrm{C}$. The crystalline phase can be re-established by assisting nucleation or cooling to very low temperatures.

\subsubsection{Structure and Phase Behaviour of $\mathrm{Cu} 3(4,6)$}

The DSC data for $\mathrm{Cu} 3(4,6)$ (Figure 5.5.8) are shown in Figure 5.5.9. For the first time for a complex, two phase transitions are observed on cooling. The sum of the enthalpies of the peaks on the cooling curve $\left(6.7 \pm 0.8 \mathrm{~kJ} \mathrm{~mol}^{-1}\right.$ and $\left.22.6 \pm 0.8 \mathrm{~kJ} \mathrm{~mol}^{-1}\right)$, approximately equals the absolute value of the enthalpy of the transition on the heating curve $\left(-29.9 \pm 0.8 \mathrm{~kJ} \mathrm{~mol}^{-1}\right)$ and the peaks are reproducible, indicating that a mesophase has likely formed. The high enthalpy of transition of the first peak on cooling suggests that the mesophase is likely to be more ordered than was observed in L3(4,6), such as a smectic or columnar mesophase.

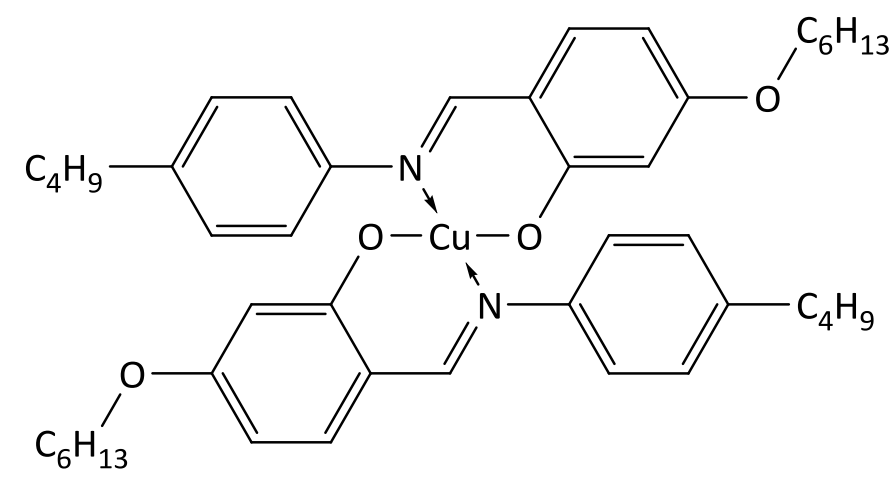

Figure 5.5.8: The structure of $\mathrm{Cu} 3(4,6)$

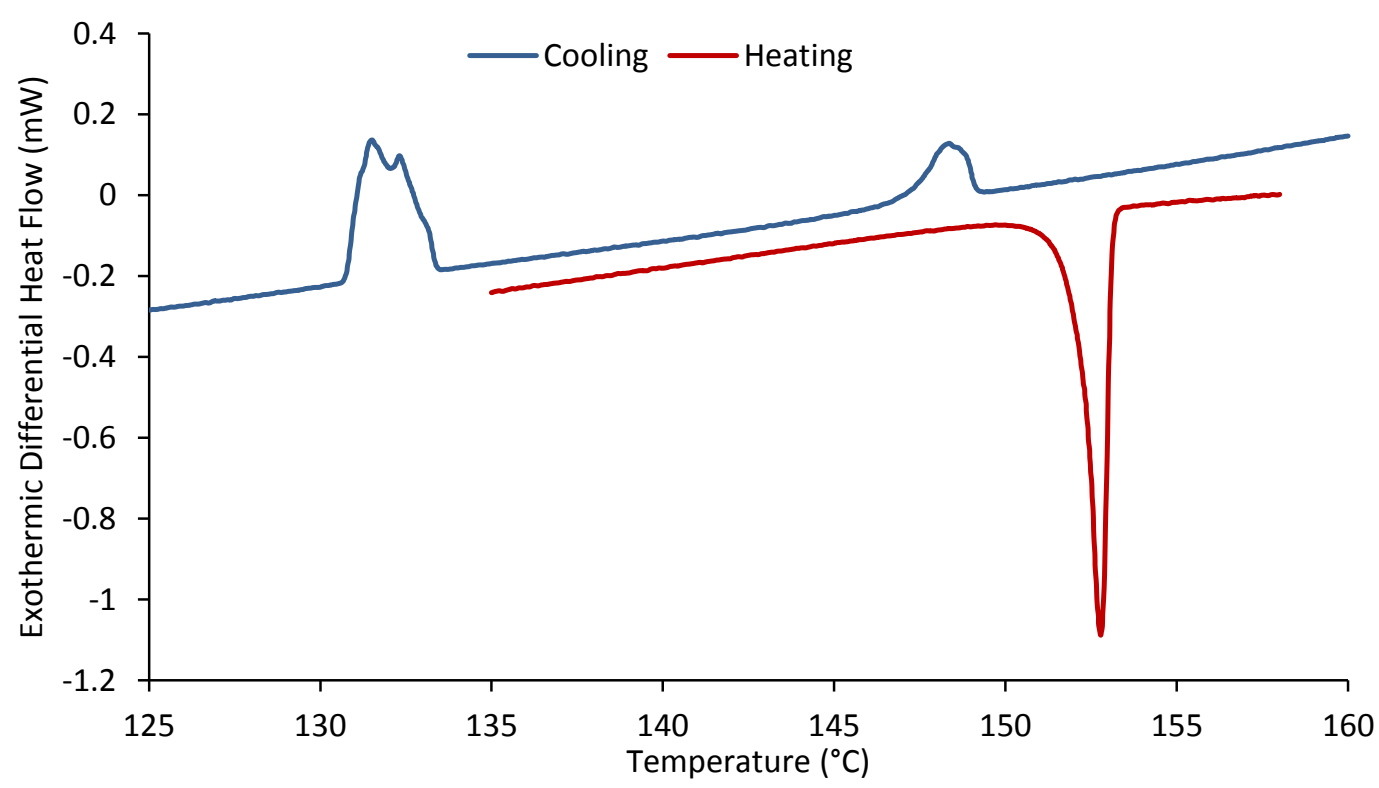

Figure 5.5.9: DSC data measured from a sample of Cu3(4,6). The temperature was varied at $\pm 1.0^{\circ} \mathrm{C} \mathrm{min}^{-1}$. 
This was confirmed with POM. Focal conic textures are observed upon cooling from the isotropic liquid phase, correlating to the formation of a smectic A mesophase (Figure 5.5.10(b) and (c)). These arise due to the complex structure of the layers at the boundaries between aligned domains in the sample. ${ }^{4}$ This assignment is consistent with the phase behaviour of similar compounds reported by Ghedini. ${ }^{56,65}$
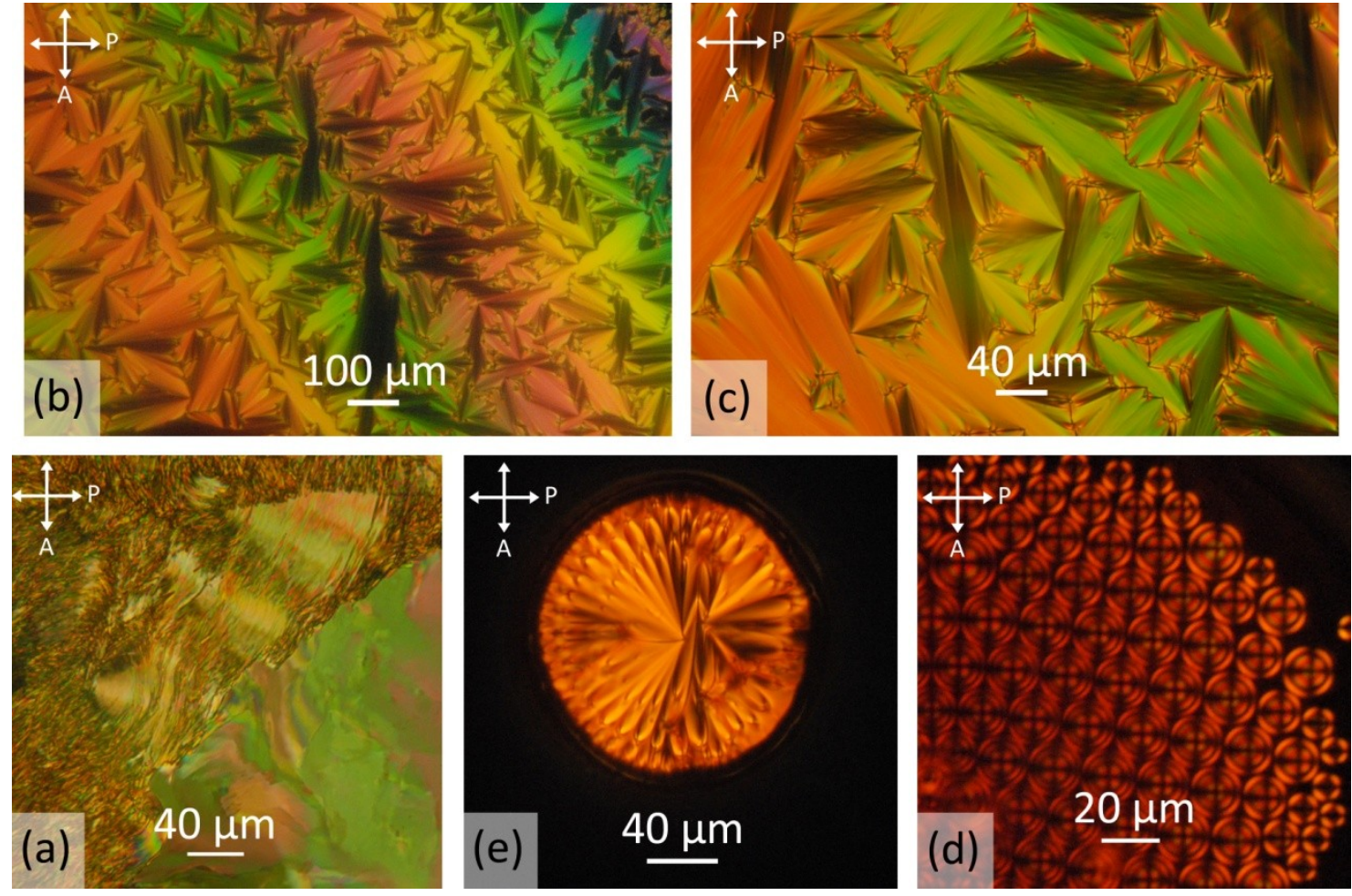

Figure 5.5.10: Examples of the optical textures formed by Cu3(4,6). POM images were captured after cooling the sample from the isotropic liquid phase to: (a) $113.3^{\circ} \mathrm{C}$; (b) $140.0^{\circ} \mathrm{C}$; (c) $144.6^{\circ} \mathrm{C}$; (d) $145.3^{\circ} \mathrm{C}$; and (e) $145.3^{\circ} \mathrm{C}$. The polariser $(P)$ and analyser $(A)$ orientations are shown.

The presence of an interface in the sample can have several effects on the texture. The first observed in Cu1 $(4,6)$ is the effect of surface tension, which results in an increase in density of the sample at the interface, and consequently the focal conic texture appears to "compress" close to the surface (Figure 5.5.10(e)) due to the resulting changes in the layer spacing and structure. ${ }^{4}$ If the reader is interested in the origin of the "flower" texture shown in Figure 5.5.10(e), Meyer has reported a theoretical study into their origin. ${ }^{66}$

Another effect is the ability for the surface to anchor aligned domains, such as in Figure 5.5.10(d). In this example, aligned domains most likely form as concentric cylinders (or tori ${ }^{66}$ ) of mesogens anchored on the surface ${ }^{4}$ and self assemble into a two-dimensional hexagonal lattice (Figure 5.5.11). The regularity of the pattern 
indicates that the domains are very uniform in size, implying a formation mechanism where domain size is limited in some manner.

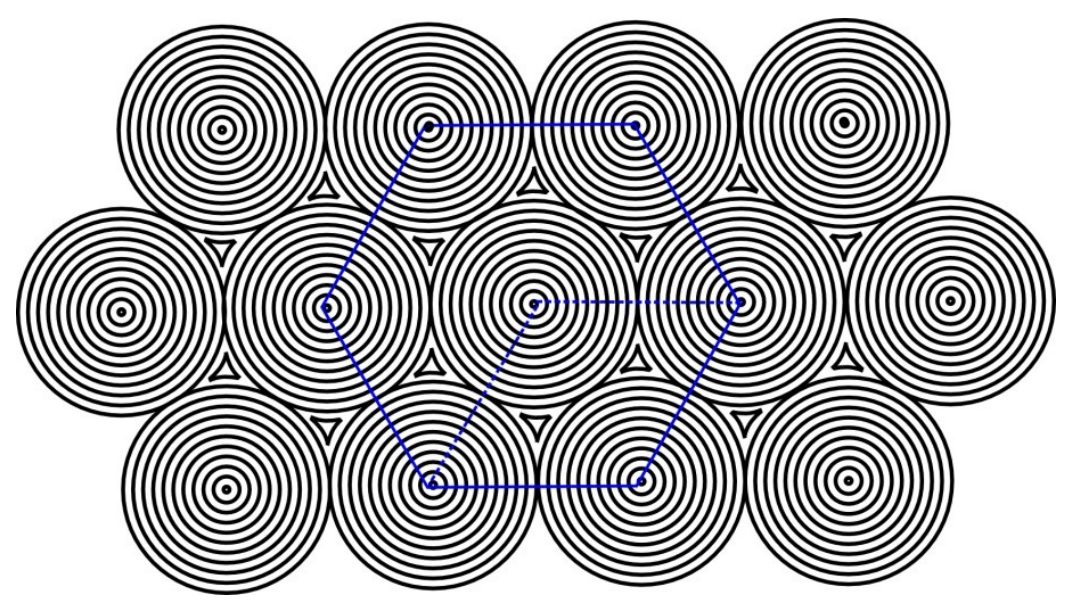

Figure 5.5.11: A schematic of the proposed structure of the smectic A mesophase in Figure 5.5.10(d), viewed along the axis of the concentric cylinders. The hexagonal packing is shown in blue, with the unit cell shown in dashed lines.

As the temperature is lowered below $130^{\circ} \mathrm{C}$ the sample briefly enters a streaky birefringent phase (Figure 5.5.10(a) - top left) before crystallising (Figure 5.5.10(a) bottom right). If cooled quickly, this streaky phase can be maintained to lower temperatures and crystallisation slowed, or stopped completely. This was also seen in the DSC data (Figure 5.5.9). This indicates a different crystalline phase or a plastic crystal of short lifetime is formed. The latter is more likely given the dependence on the cooling rate of its formation, and the fact that when reheated from this phase the sample re-establishes a smectic A mesophase, rather than melting directly to an isotropic liquid.

As with L3(4,6), SAXS and WSAXS experiments were carried out to study the structural changes in the sample as the temperature was varied. The small angle data are largely as one would expect in each phase, a single diffuse peak in the isotropic phase (Figure 5.5.12(c)), and three orders of reflection observed for both the smectic A mesophase (Figure 5.5.12(b)) and the crystalline phase (Figure 5.5.12(a)).

In the smectic A mesophase, the origins of the peaks at $2.96 \mathrm{~nm}^{-1}, 5.91 \mathrm{~nm}^{-1}$ and $9.25 \mathrm{~nm}^{-1}$ are the first, second and third order (respectively) reflections off the layers in the mesophase, corresponding to a layer spacing of $2.12 \mathrm{~nm}$ (at $145^{\circ} \mathrm{C}$ ). This layered structure is preserved into the crystalline phase, aside from thermal expansion effects (vide infra). The only noticeable difference is that the peaks are far more intense in the crystalline phase (not shown in Figure 5.5.12), showing that the layered structure in 
the crystalline phase is much more ordered than in the mesophase. This is to be expected as the layered structure in a smectic A mesophase represents an average molecular distribution in which molecules are readily exchanged between layers, rather than the strict segregation one would expect of a crystal. ${ }^{4}$
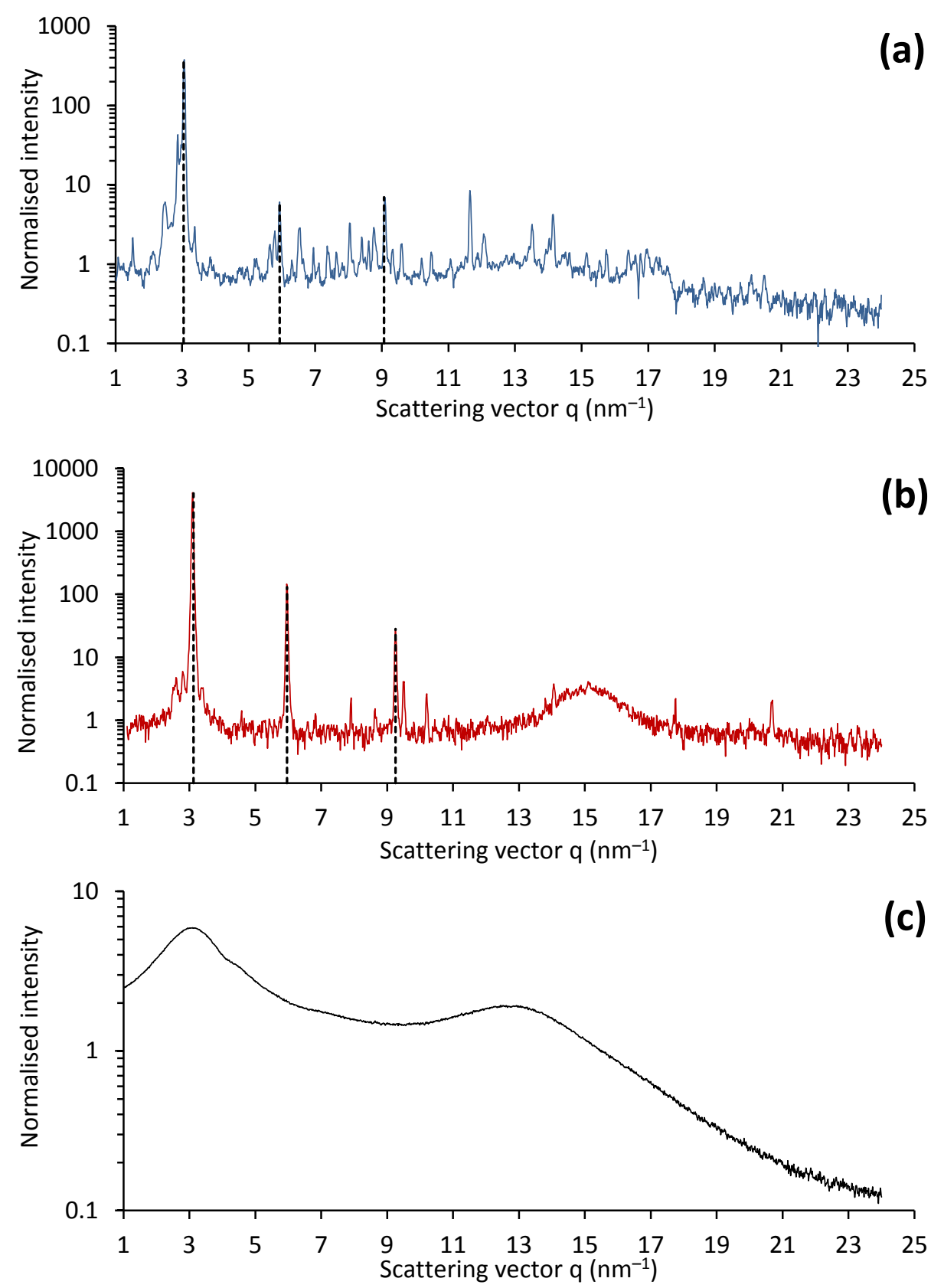

Figure 5.5.12: WSAXS data measured from Cu3 $(4,6)$ on the line collimated beamline at: (a) $115^{\circ} \mathrm{C}$ in the crystalline phase; (b) $146^{\circ} \mathrm{C}$ in the smectic A mesophase; and (c) $175^{\circ} \mathrm{C}$ in the isotropic liquid phase. The first second and third order Bragg peaks are annotated in (a) and (b). The intensities in (a) and (b) are not comparable due to differences in sample loading.

In the crystalline phase (Figure 5.5.12(a)) there is a number of sharp peaks at wide angles which, as in the crystalline phase of $L 3(4,6)$, result from scattering off planes in the crystal lattice. In contrast, the only peaks in smectic A mesophase (Figure 5.5.12(b)) are the first, second and third order Bragg reflections off the layer structure 
and a diffuse peak at wide angles from the liquid-like disorder laterally within the layers. In the isotropic liquid phase (Figure 5.5.12(c)) this diffuse peak is still present, although thermally shifted.

Upon cooling from the isotropic liquid phase, SAXS data show that $\mathrm{Cu}(4,6)$ enters the smectic A mesophase at $148^{\circ} \mathrm{C}$, consistent with the DSC data and POM observations. As cooling is continued, the peak continues to grow in intensity and increase in scattering vector (Figure 5.5.13). The former effect is due to the layered structure becoming more ordered (more uniform, fewer defects, etc.), and the latter because of thermal expansion.
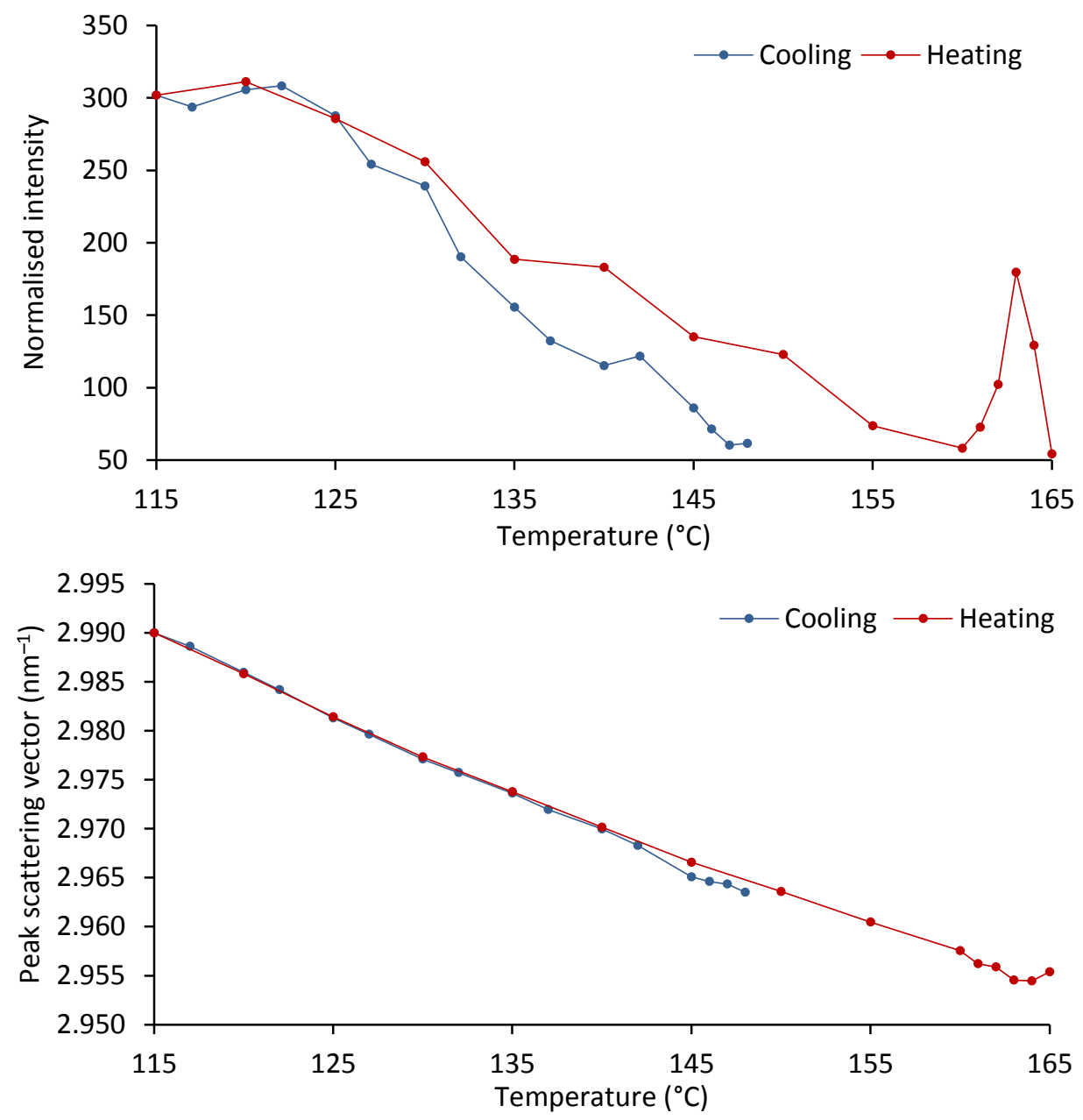

Figure 5.5.13: The SAXS peak scattering vector (bottom) and intensity (top) behaviour as the sample cools from isotropic liquid to smectic A to crystalline, and as it is heated from crystalline to isotropic liquid.

If cooling is continued the SAXS data show that the sample enters the crystalline phase between $125^{\circ} \mathrm{C}$ and $120^{\circ} \mathrm{C}$. This is signified by a plateauing of the peak intensity since, presumably, the layered structure is at its most ordered in the crystalline phase. There is, however, no discontinuity in the scattering vector of the peak, indicating that thermal expansion along the normal axis to the layers is the same in both the 
crystalline phase and the smectic A mesophase. No hint of the extra transition at crystallisation (which was indicated by DSC and POM) is observed, potentially because it does not affect the layered structure.

Upon heating from the crystalline phase, the intensity of the peak decreases, but at a slower rate than the increase in the cooling data. This is due to the fact that the sample is now in a crystalline phase, not a smectic A mesophase, so it is inherently more ordered. Unlike the cooling curve, though, there is a sharp rise in peak intensity at the melting point. This may be indicative of the brief formation of a mesophase on heating.

In contrast, the heating scattering vector data are very similar to the cooling data (except near the clearing point), showing that the layer spacing in the smectic $A$ mesophase is exactly the same as in the crystalline phase at the same temperature. This in turn means that the interactions between the layers are of the same magnitude at the same temperature whether the sample is in the smectic A mesophase or the crystalline phase. Using these data we can monitor the change in the layer repeat distance as a function of temperature (Figure 5.5.14), which can be approximated by the linear model:

$$
d=\left[(5.07 \pm 0.23) \times 10^{-4} n m K^{-1}\right] T+(1.9061 \pm 0.0094 n m)
$$

This model predicts a repeat distance of $2.054 \pm 0.016 \mathrm{~nm}$ at room temperature $(292 \mathrm{~K})$, which encompasses the experimentally determined value of $2.047 \mathrm{~nm}$.

Using the model, the linear thermal expansion coefficient in the normal direction to the layers can be found from ${ }^{67}$ :

$$
\alpha_{\perp}=\frac{1}{l} \frac{d l}{d T}
$$

where $\alpha_{\perp}$ is the linear thermal expansion coefficient in the direction normal to the layers, $T$ is the absolute temperature and $l$ is the length of the sample (in this case the layer repeat distance). The value for $\alpha_{\perp}$ for $\mathrm{Cu} 3(4,6)$ at room temperature was found to be $(2.48 \pm 0.11) \times 10^{-4} \mathrm{~K}^{-1}$. This is a much larger value than, for example, engineering metals like cast iron ${ }^{68}\left(1.01 \times 10^{-5} \mathrm{~K}^{-1}\right)$, and is comparable to amorphous 
polymers like rubber ${ }^{69}\left(2.78 \times 10^{-4} \mathrm{~K}^{-1}\right)$, indicating a significant degree of thermal expansion and therefore very weak interactions between layers.

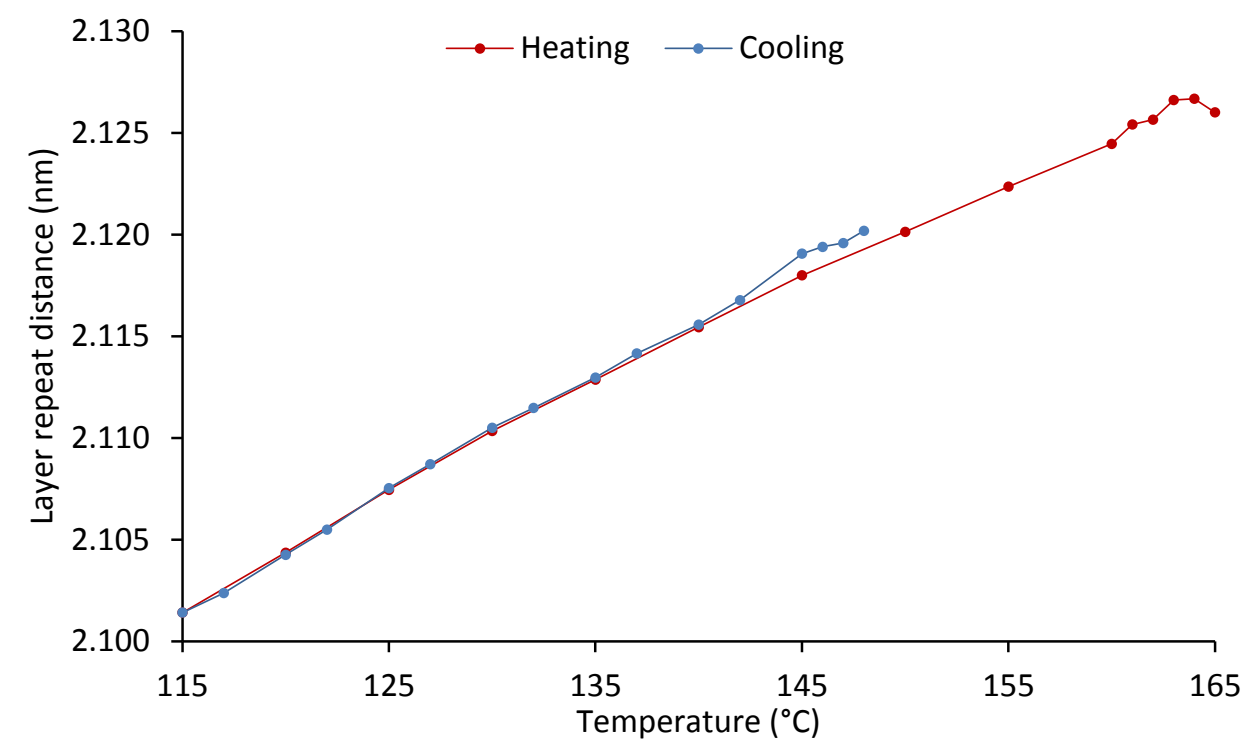

Figure 5.5.14: The dependence of layer repeat distance in $\mathrm{Cu}(4,6)$ on the sample temperature during heating in the crystalline phase and cooling in the smectic A mesophase and crystalline phase, as determined by SAXS.

Without any single crystal diffraction data it is difficult to speculate as to the exact orientation of the mesogens in the layers in the crystalline phase, and therefore the origin of the weak interaction. The spacing of the layers at room temperature $(2.047 \mathrm{~nm})$ does give some indication, though, as it is very similar to the layer spacing in Cu1 $(6,6)$ of $2.04479 \mathrm{~nm}$. This implies that the mesogens stack in the layers at room temperature in the same manner as the molecules in $\operatorname{Cu1}(6,6)$, inclined to the layer, with the repeat distance being defined by the alkoxy chains, and therefore weak dispersion forces dominate the interlayer interactions. The inclination in the crystal phase does not contradict the formation of a smectic A mesophase (instead of smectic C) as only the average alignment of mesogens in a smectic A mesophase is normal to the layer, as opposed to the alignment of individual mesogens. ${ }^{4}$

In summary, the complex crystallises in a layered structure with relatively weak dispersion forces binding the layers together. This results in a high linear thermal expansion coefficient along this axis. When heated the sample melts to an isotropic liquid at $156^{\circ} \mathrm{C}$ and when cooled from the isotropic liquid phase the sample enters the smectic A mesophase at $148^{\circ} \mathrm{C}$. In the smectic A mesophase the same relatively weak dispersion forces as in the crystalline phase maintain a layered structure, but the mesogens have liquid-like disorder laterally within the layers and the layered structure 
is less ordered. This phase is highly birefringent, producing a focal conic texture and a variety of other textures at interfaces when observed with POM. If cooled further the sample briefly enters a phase that is potentially a plastic crystal before crystallising at $132^{\circ} \mathrm{C}$.

\subsection{Conclusions}

In this chapter, the relationships between structural modification of salicylaldimines and their phase behaviour have been examined. In particular, varying the length of the alkyl tails, complexation of a mesomorphic ligand and modification of the core were studied as to their effect on the phase behaviour.

As discussed in Section 5.3, the alkyl tails play a critical role in determining the phase behaviour of the Cu1 complexes. The lengths of both alkyl tails have a significant effect on the crystal phase transition temperatures of the complexes, but not the values for the enthalpies of the transition. Furthermore, kinetic studies show that phase relaxation is a multi-step process with a pre-equilibrium and that relaxation rate is very sensitive to the length of the $\mathrm{N}$-alkyl chains. It can be concluded from this that the alkyl tails influence the crystal phase transition temperature by changing the magnitude of the kinetic barriers in the phase change process.

The tails also play a role in determining the crystal structure. In both samples examined, a layered structure is present which is defined by the length of the alkoxy chains and does not vary between phases. The phase change occurs due to the $\mathrm{N}$-alkyl chains, which likely change conformation during the crystal phase change, altering the packing. Concerning the aim of using structural modifications to control phase behaviour, modifying the lengths of the tails is a key part of controlling the crystalline phase behaviour and structure.

Complexation of a mesomorphic ligand to a metal is a common strategy employed in the synthesis of metallomesogens. ${ }^{5}$ However, the results presented in this chapter show that the mesomorphism of the ligand is not directly conferred on the complex. In this case, ligand $L 3(4,6)$ and $C u 3(4,6)$ display completely different mesomorphism to one another, the former exhibiting enantiotropic nematic mesomorphism, and the latter monotropic smectic mesomorphism. This is a consequence of the $\operatorname{Cu} 3(4,6)$ complex having far stronger lateral interactions between mesogens than the $L 3(4,6)$ 
due to the presence of the metal and the increase in the size of the core. This shows that the phase behaviour of salicylaldimine-copper complexes is not only controlled by the structure of the ligand, but also by the metal centre and the way it is incorporated into the structure.

Finally, modification of the core was shown to be the determining factor in whether the complexes were mesomorphic or not. Both the Cu1 complexes and Cu3 $(4,6)$ crystallise into layered structures. The additional phenyl ring in each ligand of $\mathrm{Cu}(4,6)$ as compared to Cu1 means that instead of directly crystallising when cooled from a liquid, a smectic A mesophase forms. The additional ring has the effect of increasing the strength of the lateral interactions between mesogens, meaning that a smectic $A$ mesophase can be stabilised. Extension of the core by forming the binuclear Cu2 complexes may have had a similar effect if it were not for their chemical instability.

It can therefore be concluded that in the salicylaldimine complexes examined in this chapter, the size of the core is the principal factor in determining whether a complex is mesomorphic or not. The shape of the core, and its interactions with other mesogens determines which mesophase forms, while the length of the tails can be use to control the phase behaviour and structure of the crystalline state. 


\section{Chapter 6}

\section{Solution Phase Photophysical Behaviour}

\subsection{Introduction}

For much of their history, the applications of liquid crystals have mostly relied on their optical properties for their use in display technology. Their use in LCDs is therefore not as a source of light, but rather as a means to alter the polarisation of light passing through them. ${ }^{36}$ Despite the LCD industry now being valued at over US\$100 billion ${ }^{9}$ and significant levels of investment being made into optimising liquid crystals for this application, all forms of liquid crystal display still operate off this basic principle. With the replacement of liquid crystals with organic light emitting diodes (OLEDs) in display technology now looking certain within the next few years, ${ }^{70}$ researchers are beginning to examine other physical properties of liquid crystals.

One area in which metallomesogens in particular have received a lot of attention is in their potential to be luminescent in the mesophase. As discussed in Chapter 3, transition metal-based metallomesogens can have a diverse range of photophysical properties in the mesophase which differ significantly from their solution or solid phase properties. ${ }^{25,41}$ In order to fully understand how the photophysical properties of a metallomesogen in a mesophase are related to the structure and other properties of the mesophase, one must begin to analyse the origins of any photophysical properties in the isolated mesogens. In particular, it must be determined which chemical moieties give rise to these properties, and how they might be affected by interactions with their environment and ultimately other mesogens.

In this chapter the solution phase electronic properties of the ligands and complexes is examined using ultraviolet-visible (UV-vis) spectroscopy. This will initially involve a breakdown of the spectral features of the ligands, their variation due to solvent effects, and the interactions between the ligands and their environments that give rise to these effects. Following this, the changes in the ligand spectra upon complexation will be examined, as well as the changes observed when the binuclear complexes are formed. The conclusions made based off the UV-vis spectra will also be compared to 
the results of density functional theory (DFT) calculations on the $L 1(6,6)$ ligand. Finally, the consequences of these results will be discussed in the context of self assembly.

The data presented in this chapter were gathered using a Varian Cary 100, dual beam, baseline corrected UV-vis spectrophotometer. All solutions were placed in quartz cuvettes for measurement in transmission mode against a solvent reference sample. The solvents used, their suppliers and purities are shown in Table 6.1.1. The computational chemistry was carried out using the Gaussian 09 program. ${ }^{71}$ Geometry optimisation was carried out using DFT with a B3LYP functional and a 6-31G++ basis set. Excited state calculations were carried out using time dependent DFT (TD-DFT) with the same functional and basis set.

Table 6.1.1: A summary of the solvents used to obtain solution phase UV-vis data from the ligands and complexes

\begin{tabular}{ccc}
\hline Solvent & Supplier & Purity \\
\hline Acetonitrile & Panreac & $99.9 \%$ \\
Chloroform & Panreac & $\geq 99.0 \%$ \\
Dichloromethane & Panreac & $\geq 99.9 \%$ \\
Ethanol (absolute) & Pure Science & $\geq 98.85 \%$ \\
n-Hexane & Labscan & $99 \%$ \\
Methanol (dry) & Panreac & $99.8 \%, \leq 0.005 \% \mathrm{H}_{2} \mathrm{O}$ \\
\hline
\end{tabular}

\subsection{Spectral Features of Ligands}

Two different classes of ligands were synthesised in this project, the $\mathrm{N}$-alkyl salicylaldimine ligands $\mathrm{L} 1$, and the $\mathrm{N}$-(4-butylphenyl) salicylaldimine ligand $\mathrm{L} 3(4,6)$. Each class shows different spectral features and therefore will be examined independently in this section. Furthermore, a range of L1 ligands were synthesised with a variety of alkyl chain lengths. The variation in chain length has a negligible effect on their spectral properties so it is only necessary that one L1 ligand be examined. $\mathrm{L} 1(6,6)$ was chosen to be the exemplar ligand of the $\mathrm{L} 1$ series.

As shown in Figure 6.2.1, each of the three bands in the spectrum of $L 1(6,6)^{*}(A, B$ and C) show strong solvent dependence, particularly on the hydrogen-bonding character of the solvent. Comparing, initially, the spectra in hexane and methanol; band A is very weak in hexane and strong in methanol, accounting for the nearly colourless vs. bright yellow appearance of the respective solutions. Band B shifts to higher energies and

\footnotetext{
* There are other transitions in the spectrum at lower wavelengths. They show no solvent dependence and are thought to originate from phenyl centred $\pi-\pi^{*}$ transitions. As they show no variation with solvent or structural modification they will not be discussed further.
} 
intensities between hexane and methanol, while band $\mathrm{C}$ shifts to significantly shorter wavelengths and lower intensities. These shifts are much smaller (although present) in the spectrum of the acetonitrile solution, indicating that the hydrogen-bonding character of the solvent is of much greater importance than the polarity, as acetonitrile is slightly more polar than methanol (dielectric constants of 38 and 33 respectively ${ }^{72}$ ).

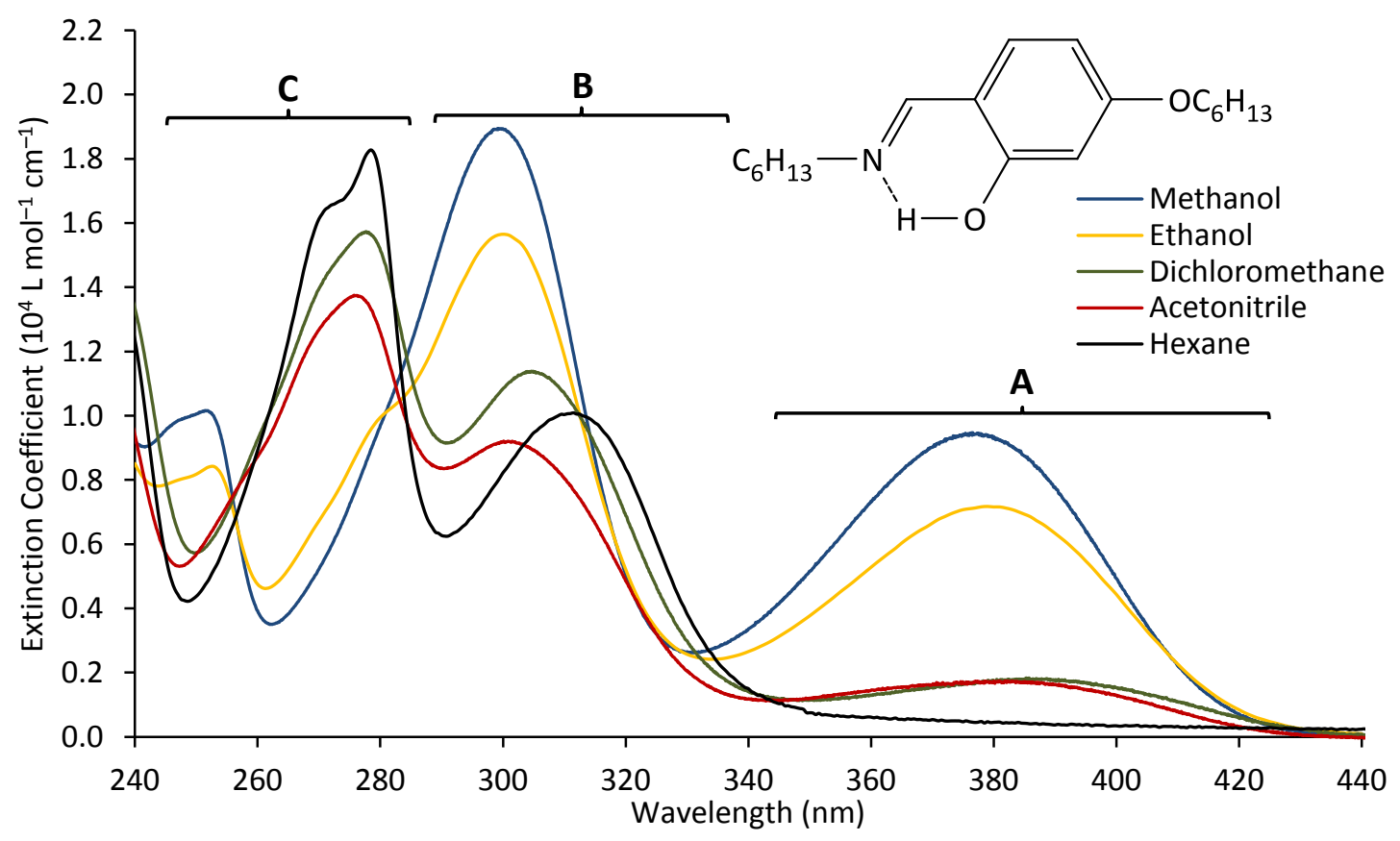

Figure 6.2.1: UV-vis spectra of $L 1(6,6)$ in a range of solvents with bands $A, B$, and $C$ annotated. The structure of L1(6,6) is shown for reference.

This conclusion is supported by the fact that the shift in acetonitrile is similar to dichloromethane, which is significantly less polar than acetonitrile, with a dielectric constant of $9.1 .^{73}$ The similarity of the shifts in acetonitrile and dichloromethane may relate to their ability to act as hydrogen-bond acceptors rather than their polarity since hexane is only slightly less polar than dichloromethane with a dielectric constant of $1.9,{ }^{73}$ but the spectrum in hexane has very different spectral features. Furthermore the ethanol peak shift is only slightly less than methanol as ethanol has less hydrogenbonding character than methanol, despite being much less polar than acetonitrile (dielectric constant of $25^{72}$ ).

The magnitude of the peak shifts and their dependence on the hydrogen-bonding character of the solvent suggest a structural change involving the imine moiety and the phenol hydrogen. The ${ }^{1} \mathrm{H}$ NMR data presented in Chapter 4 indicate that there is a strong intramolecular hydrogen-bond between these chemical functionalities due to a 
large chemical shift and significant peak broadening of the peak assigned to the phenol proton. The lower stretching frequency and breadth of the $\mathrm{O}-\mathrm{H}$ bond in the FTIR spectra further supports this and also indicates a weakening of the $\mathrm{O}-\mathrm{H}$ bond as a result.

These data all indicate the potential for the tautomeric equilibrium shown in Figure 6.2.2 to be established in solution. This phenomenon is well documented in other salicylaldimine-based compounds, which have also been shown to exhibit different absorption characteristics depending on the hydrogen-bonding character of the solvent. ${ }^{74}$ It has been proposed by Hammud et al. that this behaviour is due to hydrogen-bonding solvents increasing the acidity of the phenol proton, ${ }^{74}$ therefore shifting the position of the tautomeric equilibrium more towards the keto-amine tautomer. If this is the case with the L1 ligands, then one would expect to see elements of both tautomers in the spectrum; present in the equilibrium levels determined by the hydrogen-bonding character of solvent.

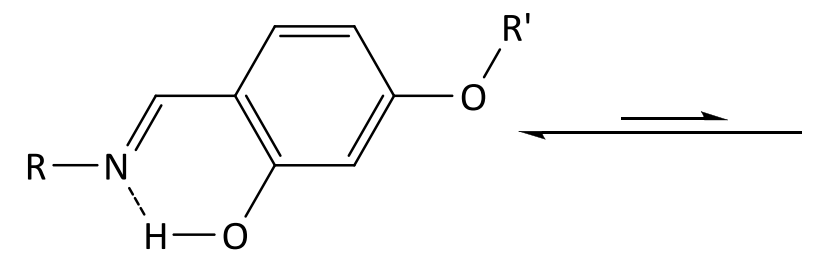

enol-imine

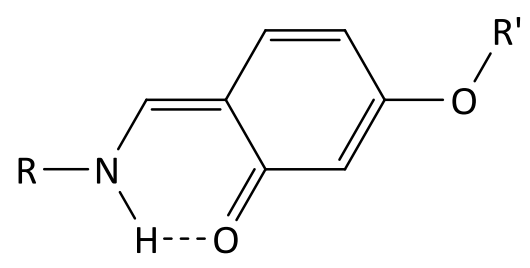

keto-amine

Figure 6.2.2: The tautomerization equilibrium between the enol-imine and keto-amine tautomers of the L1 ligands.

Of the solvents shown in Figure 6.2.1, hexane has no hydrogen-bonding character, so in solution only the enol-imine tautomer will be present. Conversely, methanol has the most hydrogen-bonding character of the solvents and would therefore contain the largest proportion of the keto-amine tautomer. When one compares the methanol and hexane spectra it is hard to identify any common features, although it is conceivable that they could be masked by the breadth of the peaks. However, when one considers the ethanol spectrum (which has slightly less hydrogen-bonding character than methanol) there is a shoulder at $282 \mathrm{~nm}$ which could correspond to the enol-imine tautomer band $\mathrm{C}$. To investigate this further, a titration experiment was carried out between ethanol and hexane (Figure 6.2.3). 


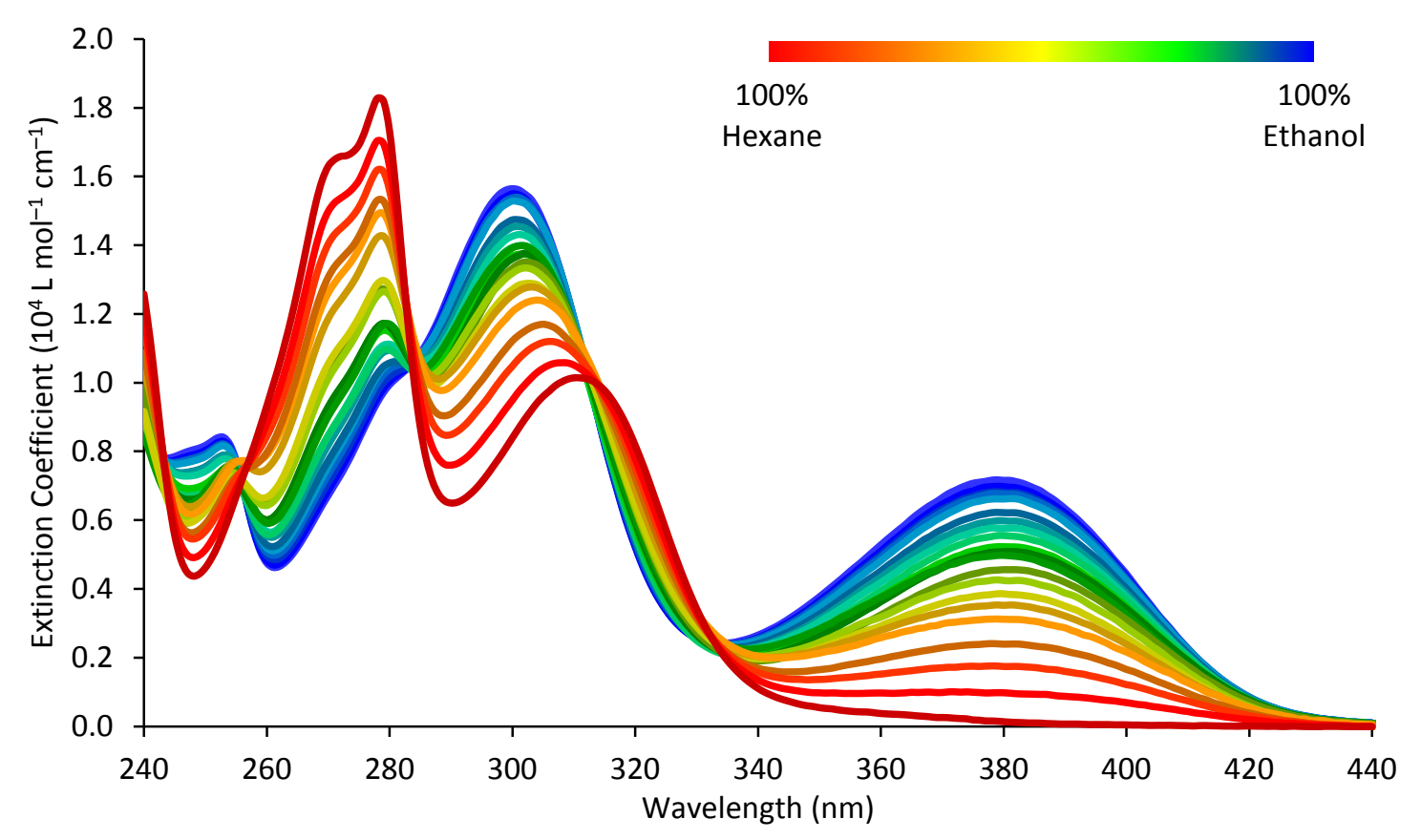

Figure 6.2.3: Titration study of $L 1(6,6)$ where the solvent is varied from $100 \% \mathrm{v} / \mathrm{v}$ ethanol to $100 \% \mathrm{v} / \mathrm{v}$ hexane.

It is evident from these data that the shoulder observed at $282 \mathrm{~nm}$ originates from the band $\mathrm{C}$ peak associated with the enol-imine tautomer, and that the keto-amine band $\mathrm{C}$ is also present at $255 \mathrm{~nm}$. Hence both species are present in the ethanol solution. Since accurate peak integrations are not possible, an estimate of the mole fraction can be obtained from the peak intensities. On this basis, the mole fraction of the ketoamine tautomer in ethanol is $45 \%$. If the shift in band $C$ were due to the solvent affecting the energy of the ground or excited states of the transition, then one would expect a gradual progression of the peak from $280 \mathrm{~nm}$ to $255 \mathrm{~nm}$, not the growth of one peak at the expense of the other as we see here. These observations can be rationalised if band $C$ is assigned to a $\pi-\pi^{*}$ transition delocalised over the conjugated system. The reduction in wavelength and extinction coefficient would be consistent with the formation of the keto-amine tautomer as this would reduce the extent of conjugation. This assignment is consistent with reported studies of other salicylaldimine-based compounds. ${ }^{54,74,75}$

The behaviour of band B is, at first glance, harder to rationalise by this means. Peaks in this area and of this intensity in salicylaldimine compounds' spectra are generally assigned to a $\pi-\pi^{*}$ transition localised on the imine $C=N$ chromophore. ${ }^{54,74,75}$ This peak would therefore be expected to decline in intensity as the proportion of the enol-imine tautomer is reduced by the addition of a hydrogen-bonding solvent. This explanation is not consistent with the data presented in Figures 6.2.1 and 6.2.3. As can be seen in 
Figure 6.2.3, the peak increases significantly in intensity, and shifts to lower wavelength with increasing ethanol fraction. These observations are summarised in Figures 6.2.4 and 6.2.5.

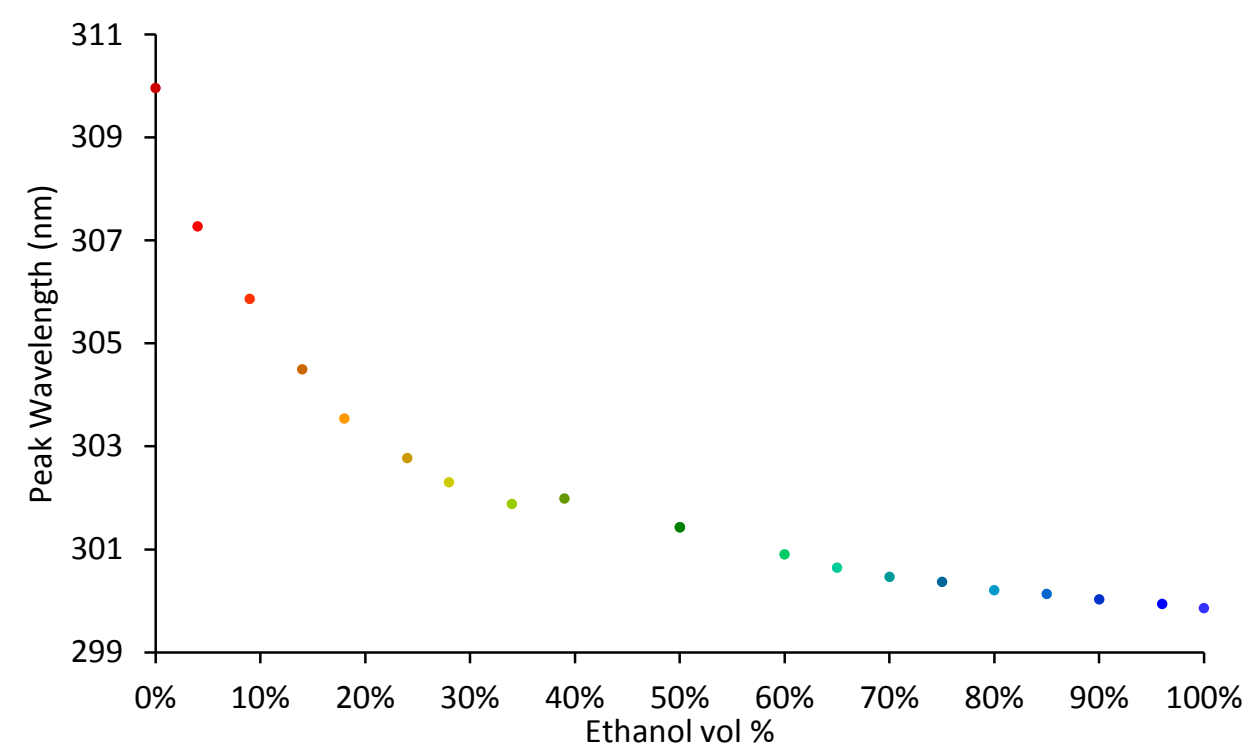

Figure 6.2.4: The band B peak wavelength data from the ethanol-hexane titration. Please see the text for an explanation of the discontinuity.

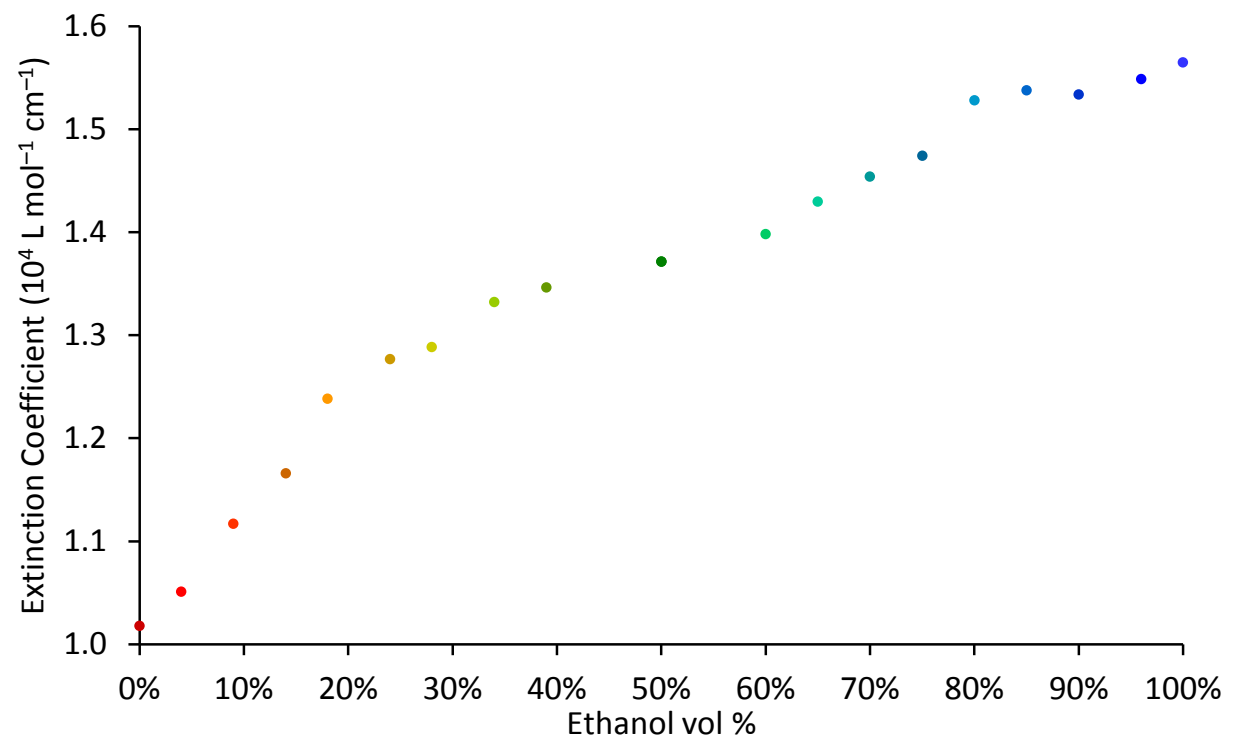

Figure 6.2.5: The band B peak extinction coefficient data from the ethanol-hexane titration. Please see the text for an explanation of the discontinuity.

The inconsistency between the predicted decline in band B and what is actually observed can potentially be explained when one considers the chromophores that are present in the keto-amine tautomer, in particular the ketone formed at the 2 position on the ring. If the $\pi-\pi^{*}$ transition of the ketone is more strongly absorbing than the imine in the enol-imine tautomer, and has a peak wavelength slightly below that of the imine, then the two absorbances would appear as a single peak, especially when the 
peaks are broad. The apparent growth of the peak with increasing ethanol volume fraction would then be as expected.

In order to ascertain if the peak wavelength and intensity data conform to this interpretation, a simple model of two overlapping parabolic peaks that scale linearly with concentration was used. The parabolic peak model used for each species was:

$$
f(\lambda)=n\left[-a\left(\lambda-\lambda_{\text {peak }}\right)^{2}+c\right] \quad \text { for }: f(\lambda) \geq 0
$$

Where $n$ is the mole fraction of the relevant species, $a$ and $c$ are arbitrary factors that control the peak breadth and intensity, $\lambda_{\text {peak }}$ is the peak wavelength and $\lambda$ is the wavelength. The sum of two closely spaced functions was computed as a function of varying mole fraction up to $45 \%$, the mole ratio of the keto-amine tautomer present in ethanol estimated from the ratio of the intensities of band $C$ in ethanol and hexane. The results of this model are plotted in Figures 6.2.6 and 6.2.7. The trend exhibited in peak wavelength is very similar to that observed in the titration data (Figure 6.2.4). However there are some differences in the trends in intensity (Figures 6.2 .5 and 6.2.7) which most likely result from deficiencies in the model.

Based on this analysis and the behaviour of band $C$, it can be concluded that band $B$ originates from the $\pi-\pi^{*}$ transitions localised on the $C=N$ imine bond in the enol-imine tautomer and the $\mathrm{C}=\mathrm{O}$ ketone bond in the keto-amine tautomer.

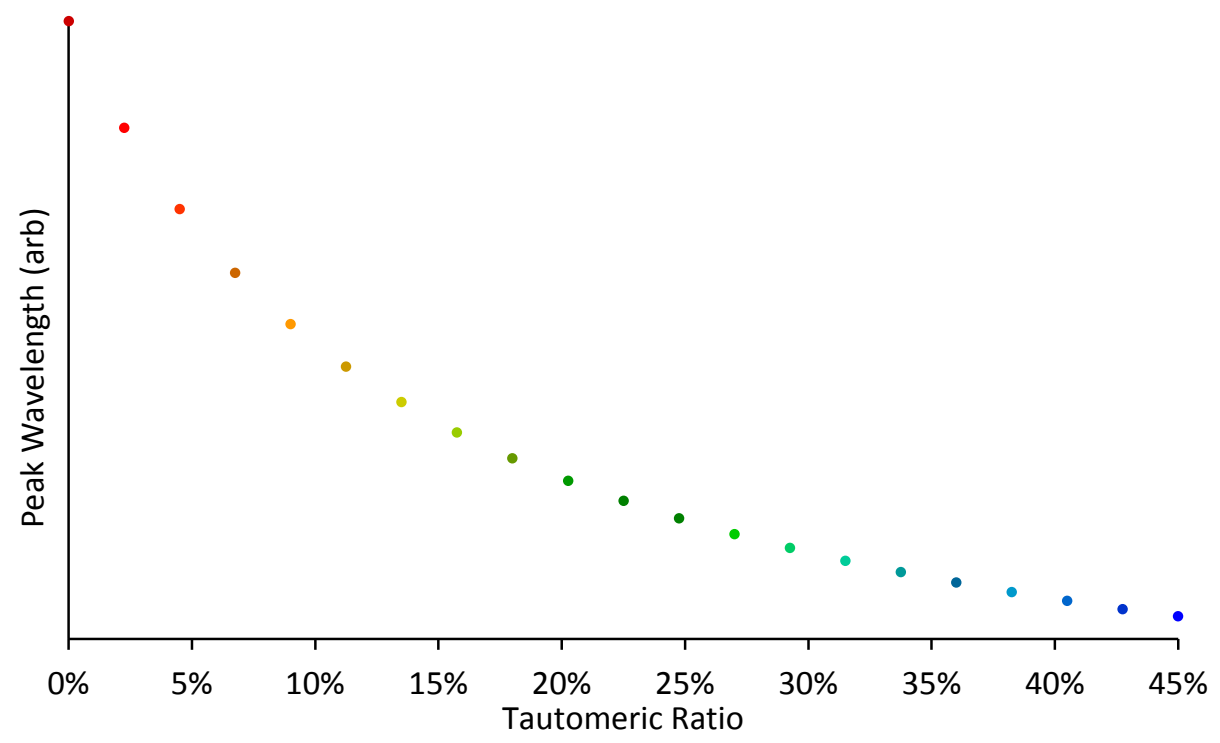

Figure 6.2.6: The trend in composite peak wavelength observed in the model as the tautomeric ratio is increased. 


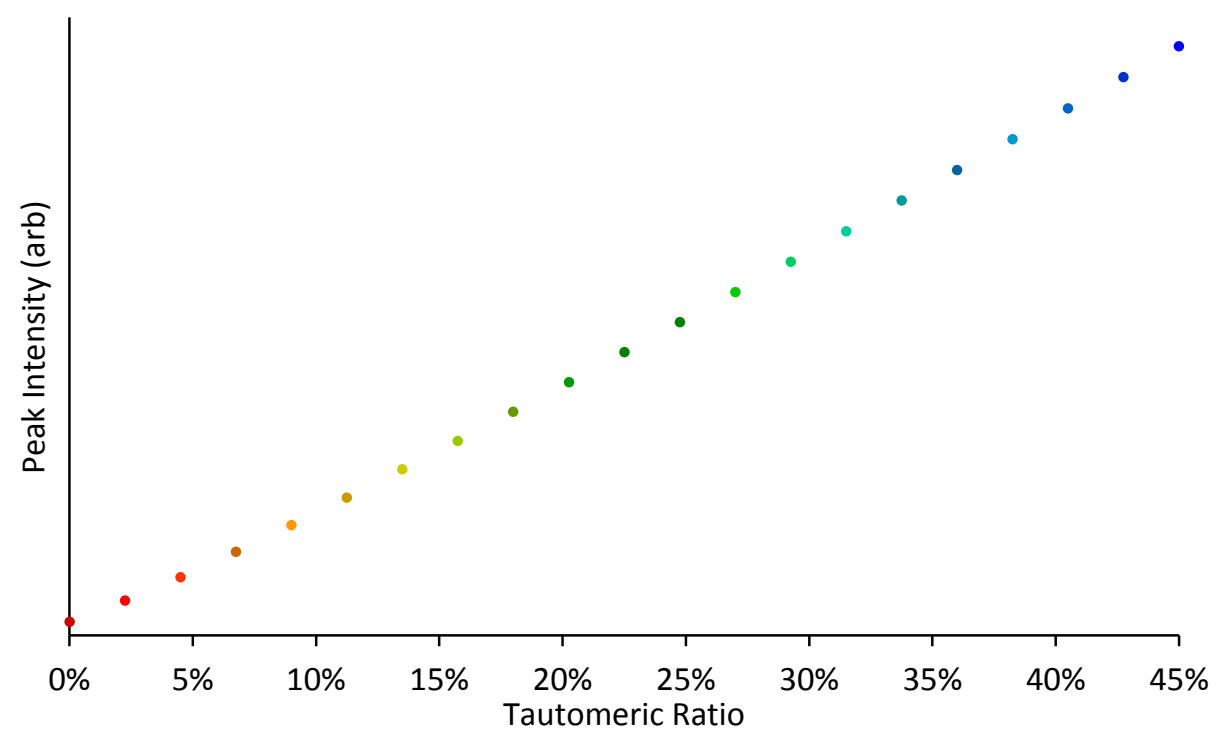

Figure 6.2.7: The trend in composite peak intensity observed in the model as the tautomeric ratio is increased.

Turning now to band $A$. This band is strongly affected by the hydrogen-bonding character of the solvent (Figures 6.2.1 and 6.2.3), and is therefore related to the tautomerism of the ligands. There has been a number of studies of the photochromism of salicylaldimine-based compounds ${ }^{74,76}$ in which tautomerization from the enol-imine tautomer to the higher energy keto-amine tautomer is induced by irradiation with ultraviolet radiation. Band $\mathrm{A}$ would be consistent with such a photochromic charge transfer as it is similar in wavelength to photochromic charge transfer bands in similar compounds, ${ }^{76}$ and because of its strong dependence on the hydrogen-bonding ability of the solvent.

The electronic absorption of the two tautomers of $L 1(6,6)$ was modelled using TD-DFT (Figure 6.2.8). The calculated UV-vis spectrum of the enol-imine tautomer shows bands that can be correlated to bands $B$ and $C$ in the spectrum of $L 1(6,6)$ in hexane at $297 \mathrm{~nm}$ and $270 \mathrm{~nm}$ respectively. Likewise, in the calculated spectrum of the keto-amine tautomer, bands at $358 \mathrm{~nm}, 285 \mathrm{~nm}$ and $241 \mathrm{~nm}$ can be correlated to bands A, B and C (respectively) in the measured spectrum of $\operatorname{L1}(6,6)$ in methanol. This further supports the hypothesis that the solvent effects observed in the spectra of the L1 ligands are due to differences in the equilibrium levels of the two tautomers in solvents with different hydrogen-bonding character. 


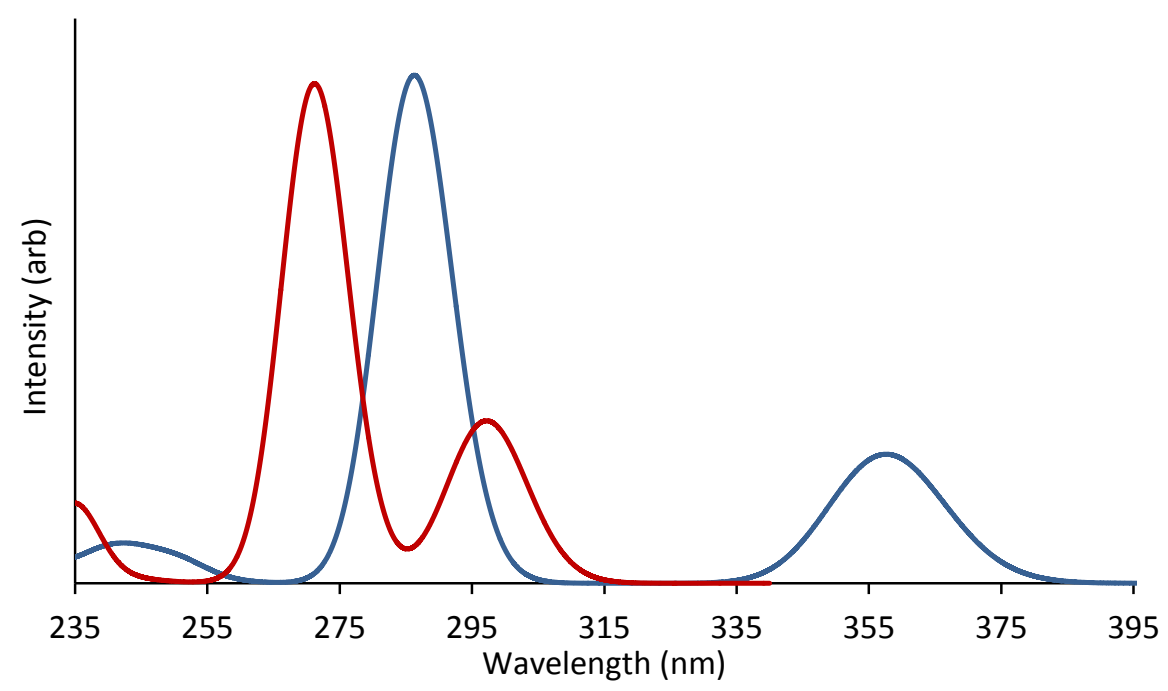

Figure 6.2.8: UV-vis spectra of the enol-imine (red) and keto-amine (blue) tautomers of L1(6,6) calculated using TDDFT

As shown in Figure 6.2.9, the UV-vis spectrum of $L 3(4,6)$ contains the same features as $L 1(6,6)$ but red shifted and with some differences in intensity. This is consistent with the extended conjugation in L3(4,6) as compared with L1(6,6). Another consequence of the extra phenyl ring in $L 3(4,6)$ is that bands $B$ and $C$ no longer show the strong solvent dependence exhibited by $\operatorname{L1}(6,6)$ (Figure 6.2.10). This is because of the increased stability of the enol-imine tautomer due to the greater conjugation, hence the tautomeric equilibrium is shifted much further towards the enol-imine tautomer. As such solvent effects are significantly reduced. The greatest effect is seen for band A, but this is still significantly reduced compared with $\mathrm{L} 1(6,6)$.

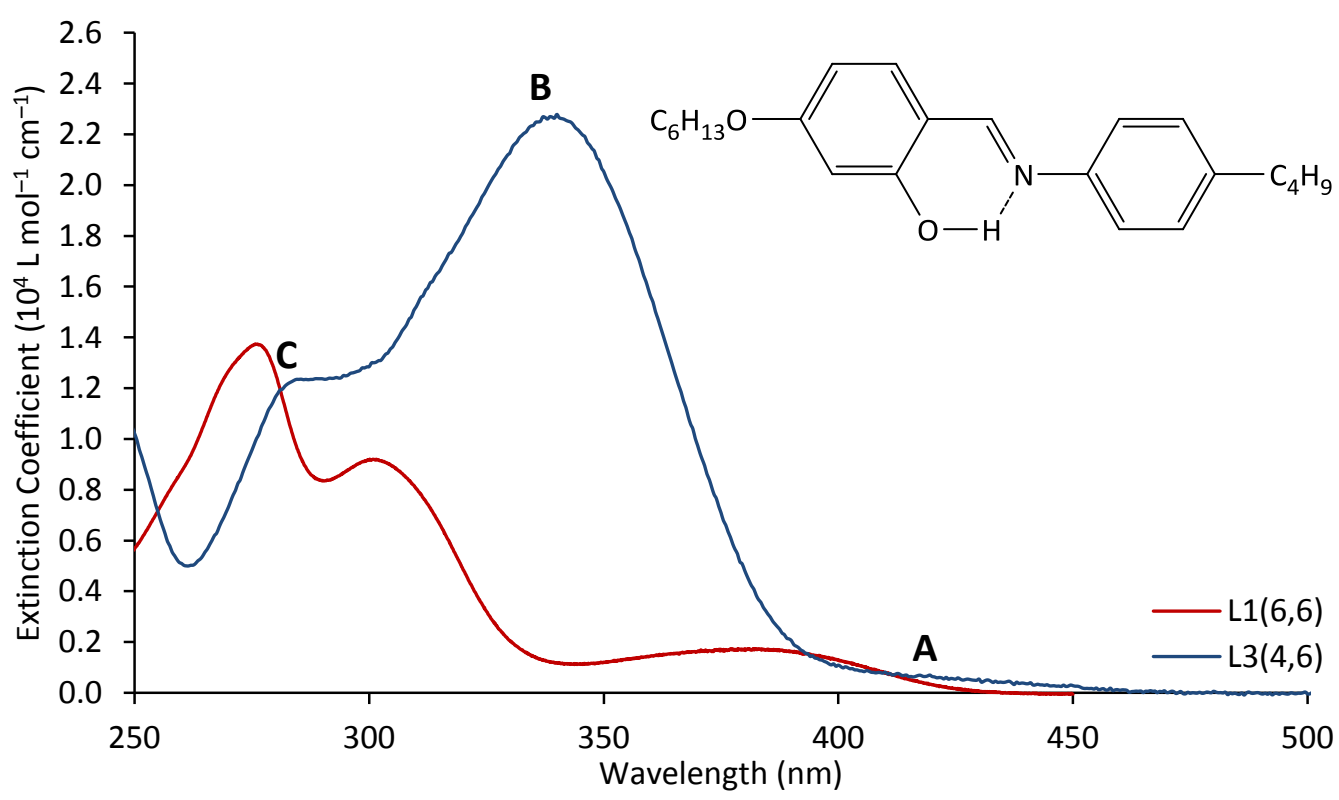

Figure 6.2.9: The UV-vis spectrum of $L 3(4,6)$ in acetonitrile with bands $A, B$ and Clabelled at $410 \mathrm{~nm}, 340 \mathrm{~nm}$ and $290 \mathrm{~nm}$, respectively. The chemical structure of $L 3(4,6)$ and the spectrum of $L 1(6,6)$ in acetonitrile are shown for reference. 


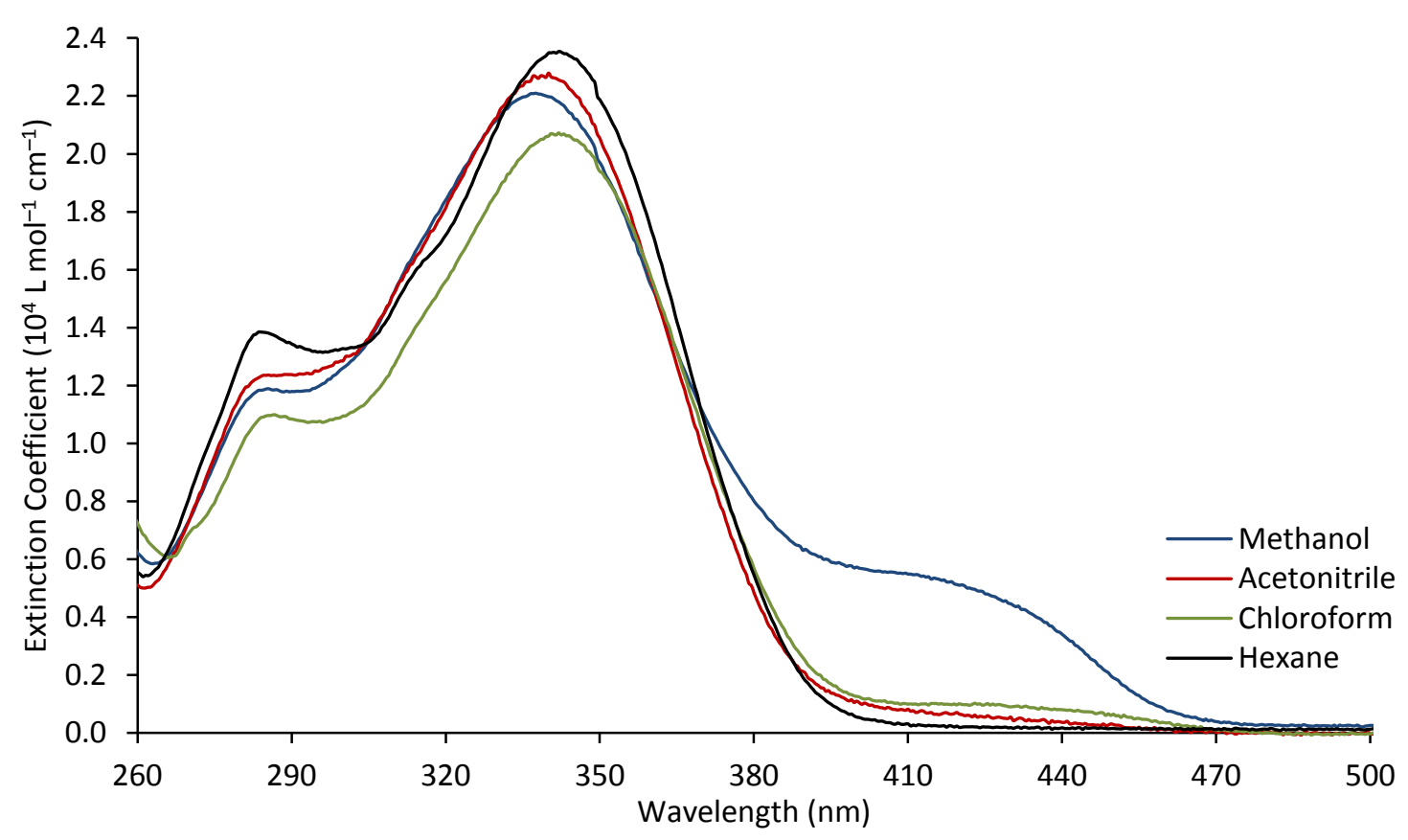

Figure 6.2.10: Electronic absorption spectra of $L 3(4,6)$ in a range of solvents.

\subsection{Spectral Features of Complexes}

\subsubsection{Mononuclear Salicylaldimine Complexes $\mathrm{Cu} 1$ and $\mathrm{Cu} 3$}

As with the ligands in Section 6.2, two classes of mononuclear complexes were synthesised, the $\mathrm{Cu} 1$ series and $\mathrm{Cu}(4,6)$. Each class will be examined separately in this section. When considering the Cu1 series of complexes, since there is little variation between Cu1 complexes with different chain lengths, one complex, Cu1(6,6) will be shown here as an exemplar for the Cu1 series.

Complexation of the L1 ligands to copper(II) had two main effects on the UV-vis spectra (Figure 6.3.1). The first is that the extinction coefficient of all peaks significantly increased. An increase in the extinction coefficients is expected, since the ligands form a 2:1 complex with copper, meaning that the number of chromophores per mole is effectively doubled upon complexation. What is unusual, though, is that for some of the peaks the intensity more than doubled upon complexation. This effect has been reported in previous studies of similar complexes by Paschke et al., ${ }^{50}$ Bhattacharjee et al., ${ }^{54}$ and Vafazadeh et al.., ${ }^{75}$ but no attempt was made to explain the results.

The other noticeable effect is the blue shifts of all the peaks upon complexation. This is reminiscent of the blue shifted peaks of $L 1(6,6)$ discussed in Section 6.2. In fact, when comparing the methanol spectra of $\operatorname{L1}(6,6)$ and $\operatorname{Cu} 1(6,6)$ (Figure 6.3.2), the reader can 
see that the wavelength of band C matches exactly, and the wavelength of band B only blue shifts by $10 \mathrm{~nm}$ on complexation. This suggests that the L1 ligands are actually in the keto-amine tautomer when bound to copper in solution, not the enol-imine tautomer which is favoured when not bound.

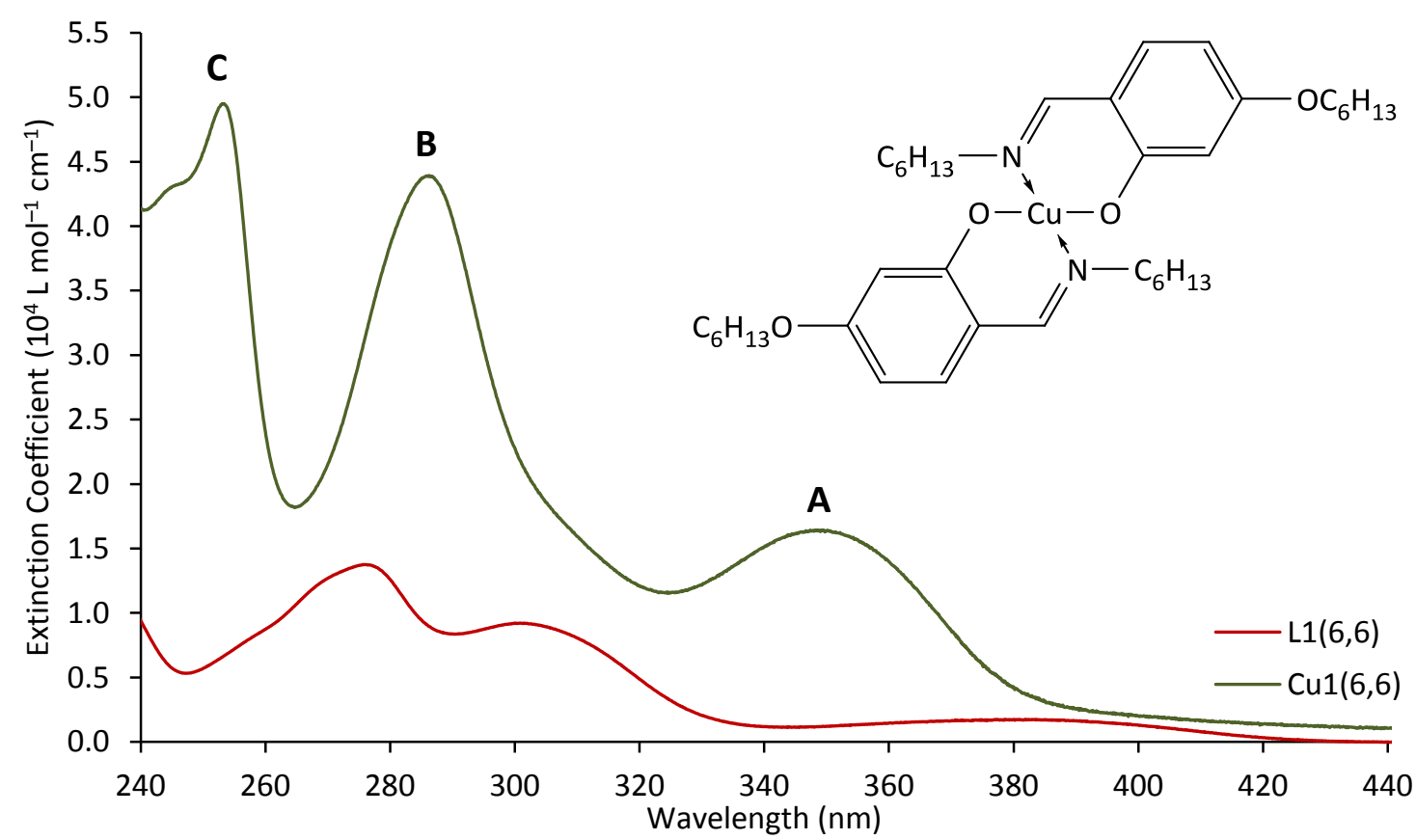

Figure 6.3.1: The UV-vis spectrum of Cu1(6,6) in acetonitrile with bands A, B and Clabelled at $350 \mathrm{~nm}, 285 \mathrm{~nm}$ and $255 \mathrm{~nm}$, respectively. The chemical structure of $C u 1(6,6)$ and the spectrum of $L 1(6,6)$ in acetonitrile are shown for reference.

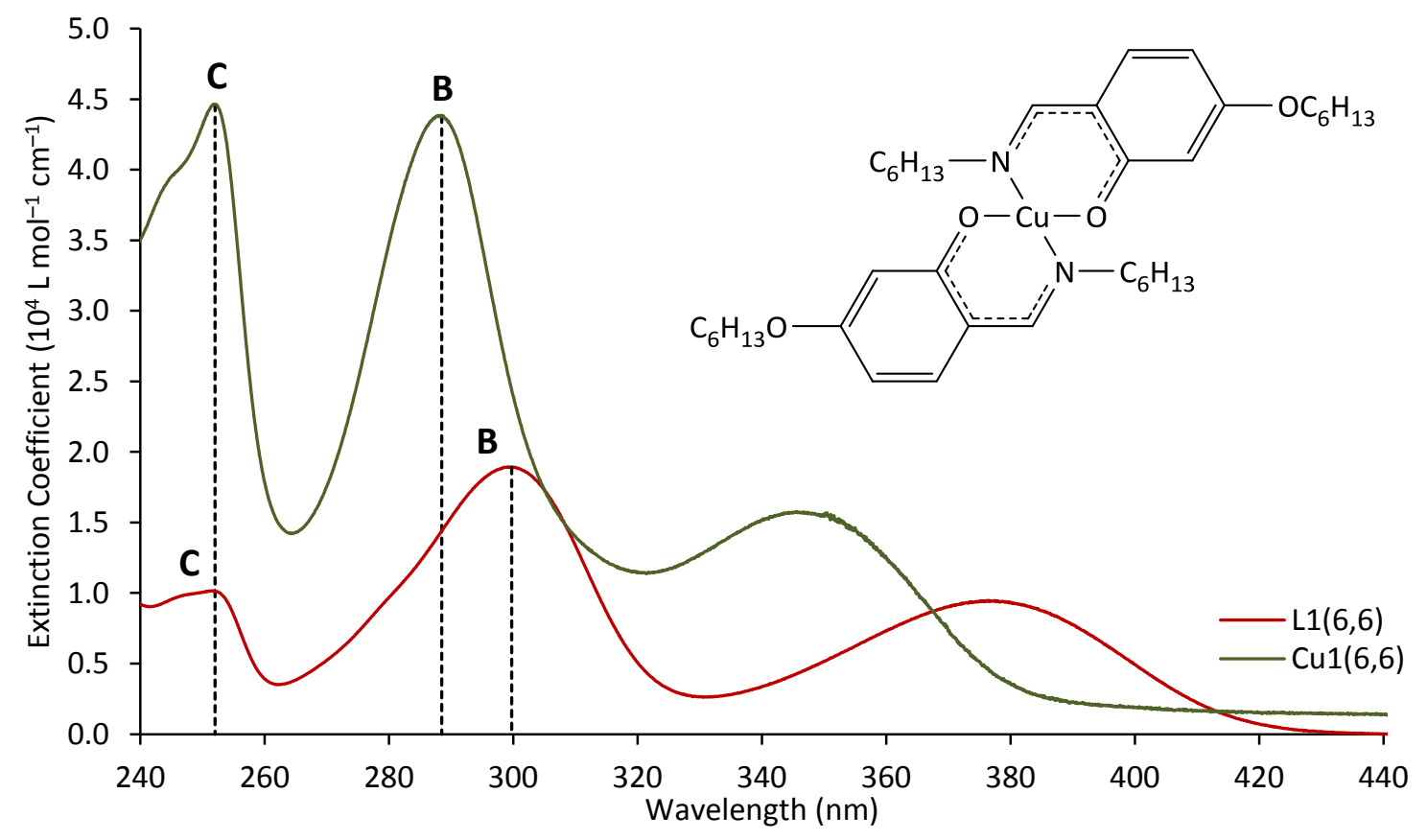

Figure 6.3.2: The UV-vis spectra of Cu1(6,6) and $\mathrm{L} 1(6,6)$ in methanol, illustrating that both spectra have the same band $C$ wavelength of $252 \mathrm{~nm}$, and shifted band B wavelengths at $289 \mathrm{~nm}$ and $300 \mathrm{~nm}$ in the Cu1(6,6) and L1(6,6) spectra, respectively. The structure of $\mathrm{Cu}(6,6)$ is also shown for reference. 
This also explains the apparent significant increase in extinction coefficient as the intensity of band $C$ in the spectrum of a pure solution of the keto-amine tautomer of L1 $(6,6)$ would be higher than band $C$ in the spectrum of a methanol solution of $L 1(6,6)$ as the latter is a mixture of both tautomers. The mole fraction of the keto-amine tautomer in a methanol solution of $\mathrm{L} 1(6,6)$ can be estimated by finding the ratio of the intensity of band $\mathrm{C}$ in methanol solution to the intensity in ethanol solution and then multiplying by the estimated mole fraction present in ethanol solution (see Section 6.2). On this basis the mole fraction of keto-amine tautomer in a methanol solution of $\mathrm{L} 1(6,6)$ is $53 \%$, and the extinction coefficient of band $C$ in the spectrum of a pure solution of the keto-amine tautomer would be $1.92 \times 10^{4} \mathrm{~L} \mathrm{~mol}^{-1} \mathrm{~cm}^{-1}$, which is only slightly less than half the intensity of band $C$ in the $C u 1(6,6)$ spectrum. The shift in band $B$ can also be explained when one considers that band $B$ in the spectrum of $\mathrm{L} 1(6,6)$ in methanol is a composite of two peaks, one from each tautomer. Therefore the position of band $B$ in the spectrum of $\mathrm{Cu} 1(6,6)$ most likely represents the position of the component of band $B$ in the $L 1(6,6)$ spectrum that arises from the keto-amine tautomer.

One apparent inconsistency with this explanation is that the crystal structure of Cu1 $(6,6)$ (Chapter 5, Section 5.3.2) shows the nitrogen to have trigonal planar geometry, with the imine bond being $1.30 \AA$ in length, as expected for a carbonnitrogen double bond. ${ }^{77}$ While this could indicate that the ligands in the crystalline phase of Cu1(6,6) adopt the enol-imine tautomer, the DFT simulation of the ketoamine tautomer shows that the nitrogen does adopt a trigonal planar geometry. This is due to the partial delocalisation of the aromatic $\pi$ system to include the amine nitrogen (Figure 6.3.3). Furthermore, the bond length of the carbon-nitrogen bond in the keto-amine simulation was found to be $1.33 \AA$, which is much closer to the expected value for an imine bond $(1.30 \AA)$ than an amine bond ${ }^{77}(1.47 \AA)$. This shows that the crystal structure of $\mathrm{Cu} 3(6,6)$ is consistent with the ligands being bound to copper in the keto-amine tautomer. A similar conclusion is reached when the enol/ketone carbon-oxygen bond is considered, as the crystal structure shows the length to be $1.32 \AA$, shorter than the expected length of $1.36 \AA$ for a phenyl-oxygen single bond. ${ }^{73}$ Moreover the carbon-oxygen bond length in the keto-amine simulation was found to be $1.27 \AA$, longer than the $1.22 \AA$ expected for a ketone. ${ }^{77}$ 


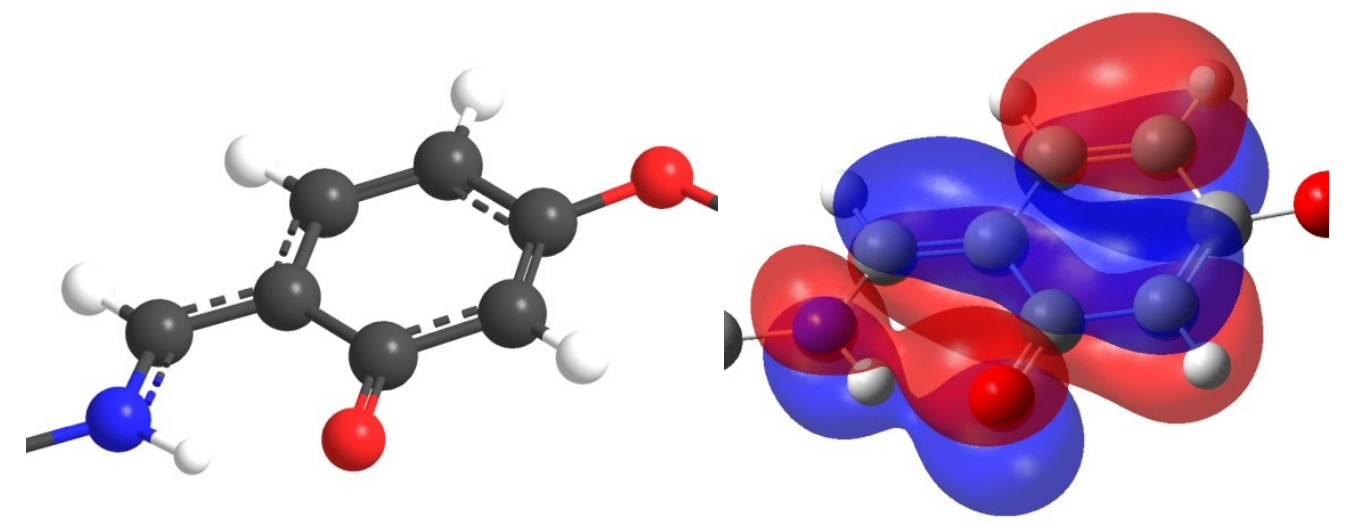

Figure 6.3.3: (left) The optimised structure and (right) the HOMO of the keto-amine tautomer of L1(6,6) with the alkyl chains omitted.

The blue shift of band $A$ is much greater than that for bands $B$ and $C$. This makes sense when one considers that in the L1 ligand, band A results from a photochromic charge transfer, exciting the molecule into the high energy keto-amine tautomer from the more stable enol-imine tautomer. It is evident that, without a proton to transfer, this tautomerism is no longer possible. Further evidence for this comes from the solvent dependence, or lack thereof, of the absorption spectra (Figure 6.3.4). Unlike the L1 absorption spectra, neither hydrogen-bonding character nor polarity significantly influences the absorption spectra of the complex. There is a slight blue shift of the peaks with increasing solvent polarity (no more than $10 \mathrm{~nm}$ ) which would be consistent with their ground states being stabilised by polar solvents.

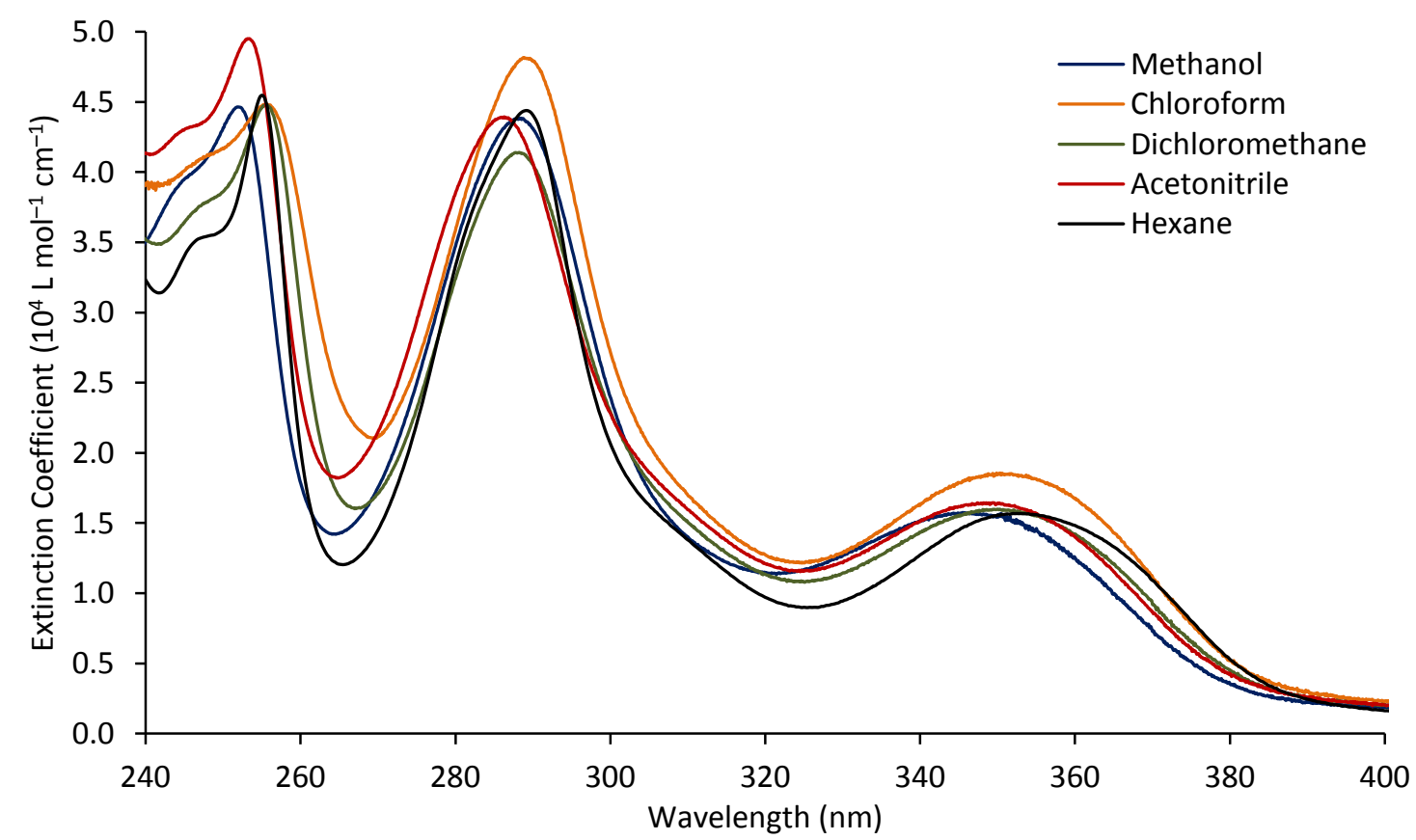

Figure 6.3.4: Electronic absorption spectra of Cu1(6,6) in a range of solvents. 
Since band $A$ in the Cu1 complex spectra does not originate from photochromism, it must be a new band resulting from complexation to copper. Given its similarity to band $A$ in the $L 1$ ligand spectra, it most likely still arises from a charge transfer, but this time involving the metal rather than a phenol proton, i.e. a ligand to metal charge transfer (LMCT) or a metal to ligand charge transfer (MLCT). This is consistent with the lack of solvent dependency as the transition does not depend on the lability of a phenol proton as in the case of the ligand. This also agrees with the studies done by Vafazadeh et al. ${ }^{75}$ and Tas et al. ${ }^{78}$ who reported that bands in this region in similar complexes resulted from LMCT or MLCT. It is difficult to determine whether the charge transfer occurs as metal to ligand or ligand to metal based off these data, but given the ligand's proclivity for charge transfer via tautomerization it seems likely it is the latter.

The changes in the $L 3(4,6)$ spectra upon complexation to copper are relatively similar to those observed in the complexation of the L1 ligands to copper in terms of the blue shift and increase in extinction coefficient for peaks $B$ and $C$ for the complex vs. the ligand (Figure 6.3.5). This is consistent with the ligands of $\mathrm{Cu} 3(4,6)$ adopting the ketoamine tautomer in solution, in the same manner as the ligands of $\mathrm{Cu} 16,6)$.

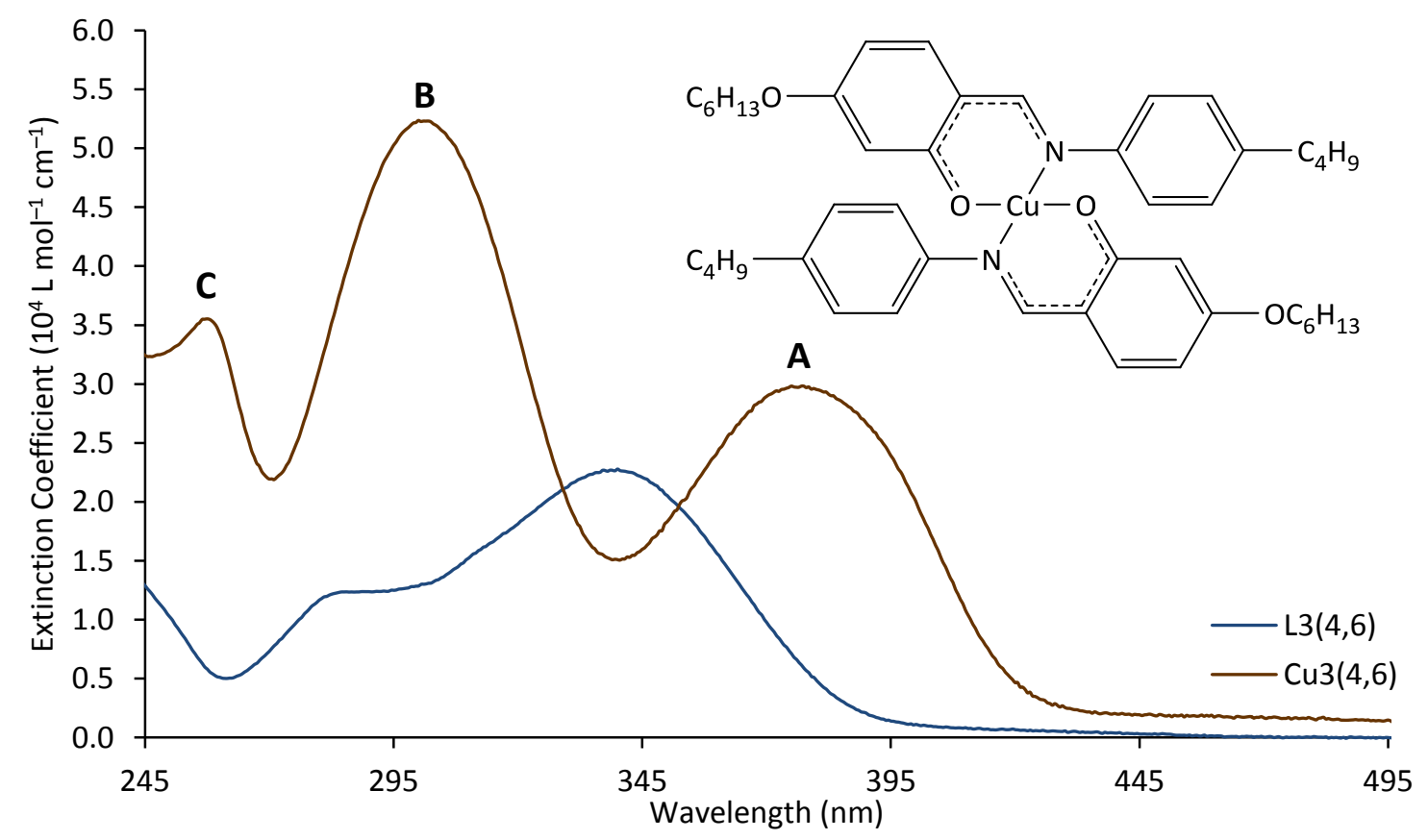

Figure 6.3.5: The UV-vis spectrum of $\mathrm{Cu}(4,6)$ in acetonitrile with bands A, B and Clabelled at $378 \mathrm{~nm}, 302 \mathrm{~nm}$ and $258 \mathrm{~nm}$, respectively. The chemical structure of $\mathrm{Cu} 3(4,6)$ and the spectrum of $L 3(4,6)$ in acetonitrile are shown for reference.

The extinction coefficient of the LMCT/MLCT band A in Cu3(4,6) is significantly increased compared with the corresponding band in the Cu1 $(6,6)$ spectrum and is red shifted by $28 \mathrm{~nm}$. Since the LMCT/MLCT transition causes a substantial redistribution 
of charge in the molecule, increasing the extent of conjugation in the molecule would be expected to have both of these effects as it would raise the probability of the transition and better stabilise the excited state via resonance.

\subsubsection{Binuclear Salicylaldimine Complexes $\mathrm{Cu} 2$}

As noted in Chapter 4, two variants of the Cu2 complexes were synthesised for this study: $\operatorname{Cu} 2(8,8)$ and $\operatorname{Cu} 2(10,6)$. As was seen with the Cu1 series, there was little variation with alkyl chain length of the complexes, hence in this section Cu2(10,6) will be used as the exemplar compound for the Cu2 series. Furthermore, when comparisons are made to the Cu1 complexes, Cu1(6,6) is used as the exemplar complex for the Cu1 series.

The spectra of solutions of $\mathrm{Cu2}(10,6)$ are shown in Figure 6.3.6. Unlike the Cu1 complexes, the spectrum of $\mathrm{Cu} 2(10,6)$ does show some solvent dependence. The spectra of solutions of $\mathrm{Cu} 2(10,6)$ in moderately polar and polar solvents (such as acetonitrile) have three bands, which are identical to the three bands present in the spectra of the Cu1 complexes (Figure 6.3.6). However, the spectra of solutions of Cu2 $(10,6)$ in highly non-polar solvents (such as hexane) have additional features that are not present in the spectra of polar and moderately polar solutions. In addition to the three bands present in the spectra of polar and moderately polar solutions, in nonpolar solutions there are additional bands at $340 \mathrm{~nm}$ and $280 \mathrm{~nm}$.

Many of the bands in the Cu1 spectra are ligand centred, and consequently should be minimally affected upon formation of a binuclear complex. In particular band $\mathrm{C}$ in the Cu1 spectra, which was assigned to a $\pi-\pi^{*}$ transition delocalised over the conjugated system of the ligand, should remain relatively unaffected by the addition of another copper centre. On inspection of Figure 6.3.6, one can see that this is the case in all solvents. Band $B$, which was assigned to a localised $\pi-\pi^{*}$ transition on either the ketone of the keto-amine tautomer or the imine of the enol-imine should be modestly affected by the formation of the binuclear complex as the electron density on the respective bond changes, while the LMCT/MLCT band $A$ should be significantly affected due to the involvement of the copper centre in the charge transfer. This is not apparent in the spectrum of a solution of $\mathrm{Cu} 2(10,6)$ in acetonitrile, indeed the spectrum is identical to the spectrum of a solution of Cu1(6,6) in acetonitrile. Moreover, these observations completely contradict those made by Paschke et al. ${ }^{50}$ 
who reported that bands $A$ and $B$ were blue-shifted by up to $30 \mathrm{~nm}$ in the spectra of similar binuclear complexes as compared to their mononuclear equivalents.

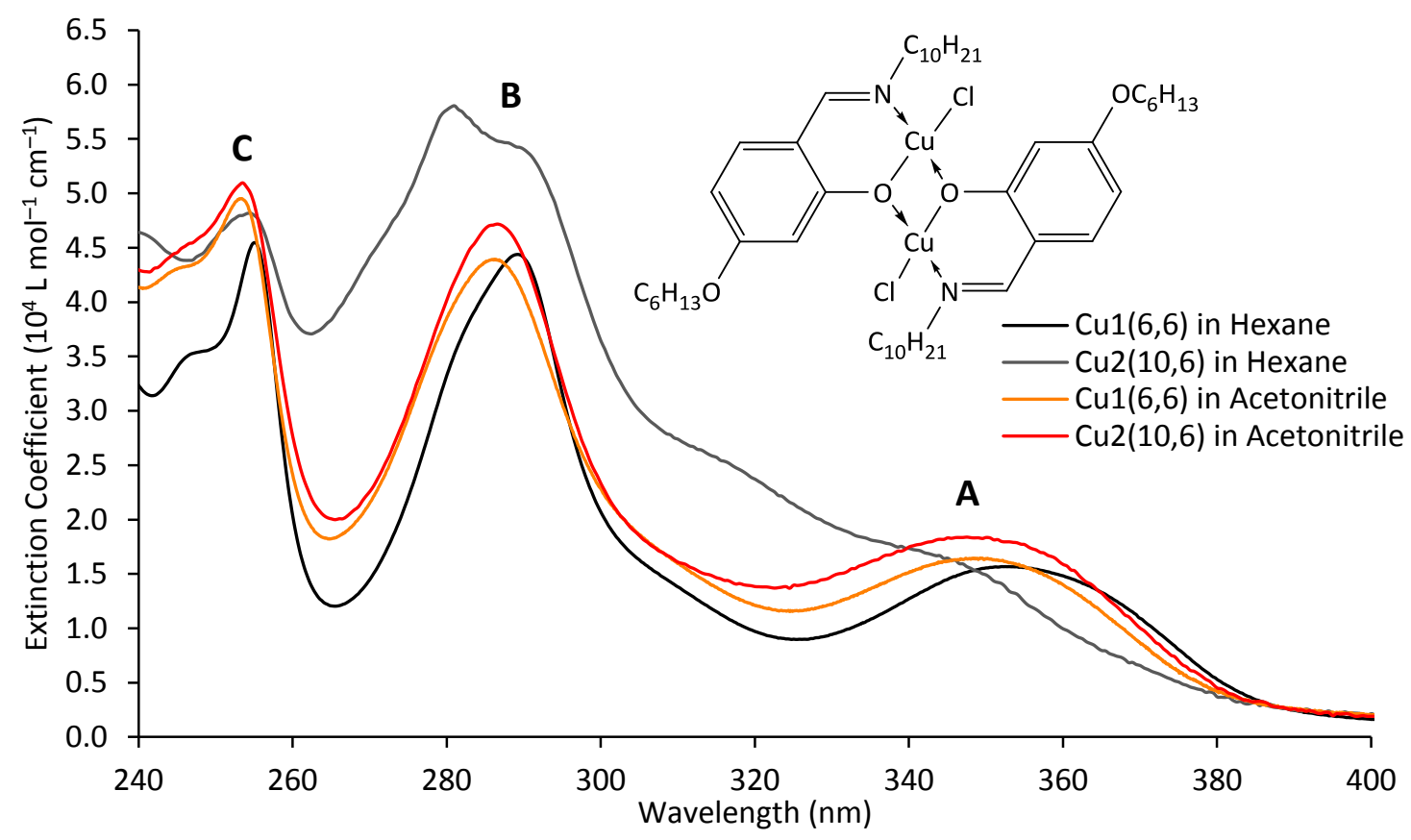

Figure 6.3.6: The UV-vis spectra of Cu1(6,6) and Cu2 $(10,6)$ in acetonitrile (orange and red) and hexane (black and grey) with bands $A, B$ and C labelled at $350 \mathrm{~nm}, 290 \mathrm{~nm}$ and $255 \mathrm{~nm}$, respectively. The chemical structure of Cu2 $(10,6)$ is shown for reference.

The extra peaks in the spectrum of the $\mathrm{Cu2}(10,6)$ in hexane are consistent with being blue shifted from bands A and B in the spectrum of Cu1 $(6,6)$ in hexane. The peak at $280 \mathrm{~nm}$ is blue shifted by $10 \mathrm{~nm}$ from band $B$ and the peak at $340 \mathrm{~nm}$ is blue shifted by $10 \mathrm{~nm}$ from band $\mathrm{A}$, which is consistent with observations reported by Paschke et al.. ${ }^{50}$ If these peaks result from the ketone/imine $\pi-\pi^{*}$ and LMCT/MLCT transitions of the binuclear Cu2 $(10,6)$ complex (respectively), then their absence from the acetonitrile solution indicates that the binuclear complex is not present in the acetonitrile solution at detectable levels. Therefore, the peaks in the acetonitrile solution spectrum must result from high levels of the mononuclear complex since the peak wavelengths and intensities in the spectrum of Cu2 $(10,6)$ in acetonitrile match those of the spectrum of Cu1(6,6) in acetonitrile. Furthermore, the presence of a peak at $290 \mathrm{~nm}$ and a shoulder at $355 \mathrm{~nm}$ in the spectrum of $\mathrm{Cu2}(10,6)$ in hexane indicates the mononuclear complex is also present in the hexane solution. This is unlikely to be due to the use of an impure product that is contaminated with the mononuclear starting material. Elemental analysis showed that the product was isolated in excellent purity and there was a clear difference in the infrared spectra of the mononuclear and binuclear complexes (Chapter 4, Section 4.4). 
As already discussed in Chapter 5, the binuclear complexes are significantly less stable than their mononuclear counterparts, in no small part due to the lability of the $\mathrm{Cu}-\mathrm{Cl}$ $\sigma$-bond. It therefore seems likely that, upon dissolution an equilibrium is established between the mononuclear and binuclear complexes (Figure 6.3.7), and that the equilibrium favours the mononuclear complex.

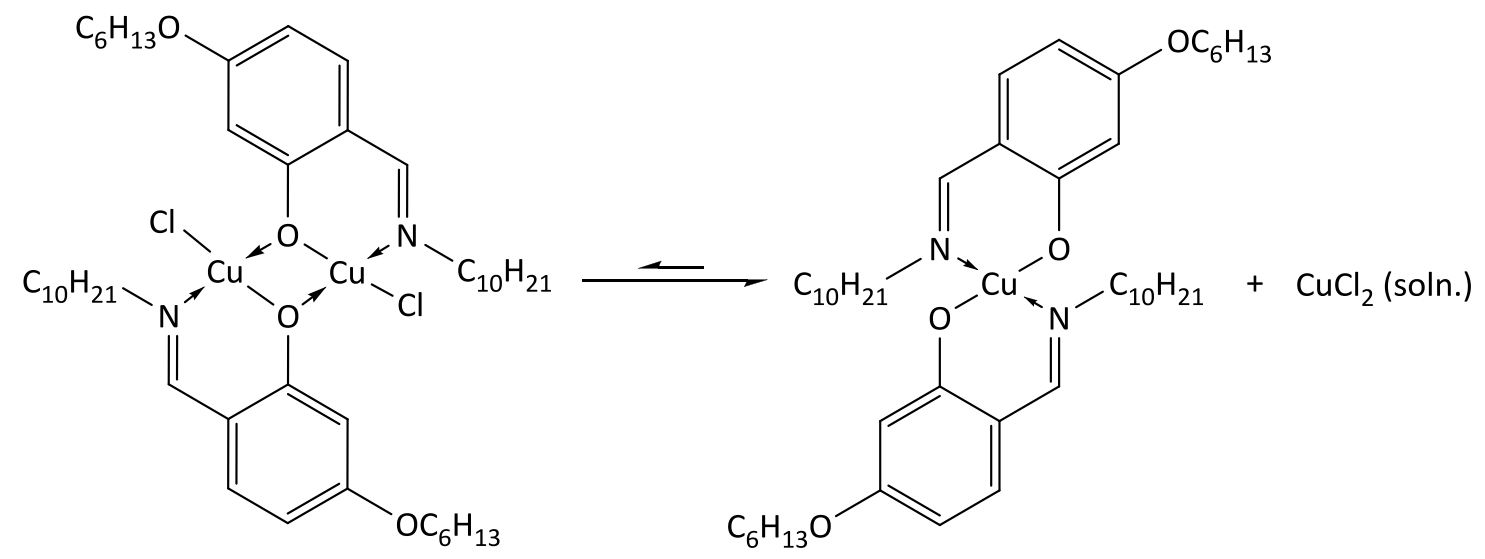

Figure 6.3.7: The equilibrium established between Cu2(10,6) and $\operatorname{Cu1}(10,6)$ when $\operatorname{Cu2}(10,6)$ is dissolved.

The position of the equilibrium is affected by the polarity of the solvent, which could be due to the greater ability of polar solvents to solubilise the copper(II) chloride byproduct. It is also likely that polar solvents that are Lewis bases are able to stabilise the transition state by coordinating to empty coordination sites on the copper metal centres in the same manner as during the synthesis (Chapter 4, Section 4.4 ) which would lower the kinetic barriers of both the forward and reverse reactions.

\subsection{Conclusions and Future Prospects}

The L1 and L3 ligands exist in a tautomeric equilibrium which enables their photophysical properties to be strongly affected by hydrogen-bonding solvents and may give rise to photochromism. The photochromism is of particular relevance to liquid crystals as there is currently a significant level of interest in the use of photochromic dopants (both mesogenic and non-mesogenic) in mesophases. ${ }^{79,80}$ It is likely that even the non-mesogenic ligands studied here would function well as dopants in such an application in structurally similar mesogenic compounds, or even into metallomesogens like Cu3(4,6).

The bands of the mononuclear Cu1 and Cu3 complexes are blue shifted from those of the free ligand and are unaffected by solvent, but show evidence of MLCT or LMCT bands. The wavelengths of the ligand-centred bands indicate that the ligands in either 
complex bind to the metal centre in solution in predominantly the keto-amine tautomer, despite the free ligand preferring the lower energy enol-imine tautomer. The presence of LMCT/MLCT bands in the complex spectra strongly suggests the possibility of luminescence. Indeed, excited states formed from absorbance in LMCT/MLCT bands can be very long lived via intersystem crossing to triplet states which decay slowly via phosphorescence. ${ }^{37,41}$ As discussed in Chapter 3 (Section 3.5) the emission of such states can be enhanced in the mesophase due to aggregationinduced phosphorescence emission (AIPE). This arises due to the structure of the mesophase restricting the motion of mesogens thereby decreasing thermal quenching, but also being less condensed than a crystal and therefore less vulnerable to concentration quenching. ${ }^{41}$

There is also the possibility of forming intermolecular excimer states across several mesogens via charge transfer between ligands on adjacent mesogens. ${ }^{42}$ This phenomenon has been observed in iridium complexes in the crystalline phase, ${ }^{42}$ but the strong interactions between copper-salicylaldimine metallomesogens in the mesophase raise the possibility of observing it in the mesophase. In addition to the $\pi-\pi$ interactions common to most mesogens, copper complexes that contain $\mathrm{Cu}-\mathrm{O}$ bonds have particularly strong intermolecular axial interactions, as demonstrated by GiroudGodquin et al. with their copper-alkanoate "lantern" complexes. ${ }^{33}$ If these interactions are strong enough, they may be able to sustain intermolecular excimer states in the mesophase.

Although only one mononuclear complex $(\mathrm{Cu} 3(4,6))$ formed a mesophase, it is possible that mesomorphism could be induced in the other mononuclear complexes via doping them into a mesophase. The Cu1 complexes could be doped into $L 3(4,6)$ or $\mathrm{Cu}(4,6)$, both of which are structurally similar to the Cu1 complexes and would therefore be likely to mix well, a challenge commonly faced in the preparation of doped systems. ${ }^{25}$ It has been reported in previous studies of the absorption and luminescence of such doped systems that the mesophase can dictate the polarisation of the emission, ${ }^{48}$ but the real benefit is that it provides a means of independently optimising the photophysical and mesomorphic properties of the system..$^{25}$ In particular, doping a non-mesomorphic metal complex into an organic mesogen (like a Cu1 complex into $L 3(4,6))$ allows mesophase formation, while preserving many of the aggregation effects 
seen in the luminescence of metallomesogens. ${ }^{25}$ In particular, with the Cu1 complexes, it would mean that their photophysical properties could be incorporated into a mesophase, despite not being able to form mesophases themselves.

Finally the binuclear complexes' instability means that they exist in equilibrium with their mononuclear equivalents in solution. Consequently, their photophysical properties were unable to be discriminated from those of the mononuclear complex they decomposed into. Improvements to the chemical stability of the binuclear complexes by use of less labile ancillary ligands, or longer tails would enable the study of their solution phase absorption properties. It is also possible that with improved chemical stability these compounds would be mesomorphic (Chapter 5, Section 5.6), and therefore absorption and emission studies could be carried out in the mesophase. In particular, the behaviour of the MLCT/LMCT band in the binuclear complexes would be of interest with respect to the lifetime of any excited states and the formation of intermolecular excimer states in the mesophase. 


\section{Chapter 7}

\section{Conclusions and Future Work}

\subsection{Conclusions}

A range of mononuclear $\mathrm{N}$-alkyl,4-alkoxysalicylaldimine copper(II) complexes (Cu1) were synthesised and analysed for their structure, phase behaviour and solution phase absorption properties. While these complexes are not metallomesogens, they do exhibit multiple crystalline phases. All of the Cu1 complexes exhibit both a high and a low temperature crystalline phase, with transition temperatures showing a strong dependence on the length of the alkyl chains. Relaxation to the low temperature phase on cooling from the high temperature phase occurs slowly and its rate is highly dependent on the relaxation temperature and the length of the $\mathrm{N}$-alkyl chain. The apparent negative activation energy of relaxation shows that there are multiple steps in the relaxation process. Both phases have layered structures, which show little variation in spacing with temperature, indicating strong interactions between the layers. Transitions between phases are thought to be due to changes in the conformation of the $\mathrm{N}$-alkyl chains within the layers which result in a substantial change in the unit cell volume.

The $\mathrm{N}$-alkylsalicylaldimine ligands exist in solution in tautomeric equilibrium between the lower energy enol-imine tautomer and the higher energy keto-amine tautomer. Exchange between the two occurs via photochromic charge transfer, as well as the position of the equilibrium being strongly affected by the hydrogen-bonding character of the solvent. In solution, when bound to copper, the ligands adopt the keto-amine tautomer, as shown by the blue shift of the ligand centred bands in the complex spectrum with respect to the ligand spectrum. The complexes also show evidence of a MLCT/LMCT band.

Structural modification of two of the mononuclear $\mathrm{N}$-alkylsalicylaldimine complexes to form the binuclear complexes $\mathrm{Cu2}(10,6)$ and $\mathrm{Cu2}(8,8)$ did not induce mesomorphism. The resulting complexes were chemically unstable, decomposing when heated to $126^{\circ} \mathrm{C}$ to at least two compounds with different melting points. The complexes were 
also not stable in solution, establishing an equilibrium with their corresponding mononuclear complex (a decomposition product) when dissolved. The position of the equilibrium is strongly affected by the polarity of the solvent with polar solvents (particularly those that are Lewis bases) favouring the decomposition products. This instability is thought to be due to the lability of the chloride ancillary ligands.

Modification of the mononuclear complex by substitution of the $\mathrm{N}$-alkyl moiety for a $\mathrm{N}$-(4-butylphenyl) moiety, thereby enlarging the core, resulted in mesomorphism in both the free ligand $(\mathrm{L} 3(4,6))$ and the copper(II) complex $(\mathrm{Cu} 3(4,6))$. The ligand formed an enantiotropic nematic mesophase between $42^{\circ} \mathrm{C}$ and $60^{\circ} \mathrm{C}$. The nematic mesophase was easily supercooled, and would form a plastic crystal phase rather than crystallising when cooled. The solution phase absorption spectrum of $L 3(4,6)$ was red shifted with respect to the $L 1$ ligands, and more intense. The $L 3(4,6)$ ligand also has a decreased tendency to tautomerise in solution. These effects are due to the extension of the conjugated system in the ligand which increases the stability of the enol-imine tautomer. Only the photochromic charge transfer band showed sensitivity to the hydrogen-bonding character of the solvent.

Complexation of $L 3(4,6)$ to copper significantly altered the phase behaviour, due to the resulting change in the core shape and therefore magnitude of interactions between mesogens in the mesophase. Cu3 $(4,6)$ forms a monotropic smectic A mesophase between $148^{\circ} \mathrm{C}$ and $131^{\circ} \mathrm{C}$. The layered structure of the smectic A mesophase is preserved into the crystalline phase. There is no change in the magnitude of the interlayer interactions between the two phases between the smectic A mesophase and the crystalline phase. Furthermore, the inter-layer interactions are much stronger in the Cu1 complexes than in $\mathrm{Cu} 3(4,6)$. This may be one of the reasons why the Cu1 complexes do not form mesophases. The $L 3(4,6)$ ligand likely binds to copper in the keto-amine tautomer, and the solution phase spectrum of $\mathrm{Cu} 3(4,6)$ has a MLCT/LMCT band.

This study has shown the sensitivity of the phase behaviour of mesomorphic compounds to small structural variations. The addition of a single phenyl ring has been shown to be a determining factor as to whether or not these complexes are mesomorphic. Furthermore, complexation of a mesomorphic ligand to a metal can completely alter the interactions between the mesogens in the mesophase, and 
therefore which mesophase is formed. This study has also shown that the electronic absorption properties of these compounds are strongly affected by changes in the structure of the core, which can in turn be influenced by the environment around the molecule.

\subsection{Future Work}

With respect to the phase behaviour and structure of the complexes and ligands synthesised in this project, several aspects require further investigation. The phase behaviour of the Cu1 complexes, particularly the kinetics of phase relaxation, is not well understood. Further kinetic and high temperature X-ray studies of the phase relaxation process will assist in determining exactly what conformational changes lead to the phase changes in the Cu1 complexes, and the role of chain length during relaxation. In addition, only one $\mathrm{N}$-(butylphenyl) derivative has been investigated in this study, namely Cu3(4,6) and its ligand $L 3(4,6)$. Expansion of the Cu3 and L3 series to include other chain lengths will provide more information about how the length of the alkyl chains affects the structure and temperature range of the formed mesophases.

The chemical stability of the Cu2 complexes must be improved if they are to be investigated further, particularly for their potential as catalysts. ${ }^{81}$ This could be done by substitution of the labile chloride ligands for a less labile alternative (such as fluoride), or through the use of a tridentate salicylaldimine ligand. ${ }^{82}$ While this would improve the chemical stability of the complexes, the ability of the complex to act as a catalyst may depend on the presence of exchangeable ligands to allow access to binding sites on the metal centre. Any development of these complexes towards their use as catalysts would need to find a balance between these two considerations.

This study incorporated an investigation of the solution phase absorption properties of the complexes and ligands. This needs to be extended to include studies of the crystalline phase and mesophase to investigate any aggregation effects that may occur. In addition, the emission spectra of the complexes and ligands should be measured, initially in solution phase but also in the crystalline phase and the mesophase. This will enable better understanding of how the electronic structures of mesogens and metallomesogens are affected by self assembly, and whether self assembly can be 
used to tune emission. The photochromism of $L 3(4,6)$ in the mesophase should also be explored to see what effect tautomerization has on the structure of the mesophase.

The final aspect that requires further investigation is the possibility of using some of the complexes or ligands synthesised here as dopants. There are two aspects to this that could be explored. The first is to use either the $L 1$ or $L 3(4,6)$ ligands as photochromic dopants into $\mathrm{Cu} 3(4,6)$ in the smectic A mesophase. This could introduce light dependence into the phase behaviour and physical properties of $\mathrm{Cu} 3(4,6)$. The second would be to dope one of the copper complexes into $L 3(4,6)$ in the nematic mesophase. This would allow examination of luminescence in the mesophase at much lower temperatures than could be achieved with a pure metallomesogen, and may result in completely different properties to both the dopant and the host with regard to aggregation effects. 


\section{Glossary}

Anisotropic Directionally dependant (not isotropic).

Birefringence The rotation of plane polarised light by a substance with multiple refractive indices (i.e. optically anisotropic).

Calamitic A rod-shaped class of mesogen.

Columnar

A mesophase in which mesogens have two dimensions of translational order and orientational order. The mesogens self assemble to form columns that can be thought of at one dimensional liquids.

Core The rigid, anisotropic moiety at the centre of a mesogen.

Director A vector describing the orientation of a mesogen.

Director field

A vector field defined by the directors of the mesogens in a mesophase.

Disclination A line defect in the director field of a mesophase.

Discotic A disc-shaped class of mesogen.

Enantiotropic

Lanthanidomesogen

Lyotropic

Mesogen

Mesogenic

Mesomorphic

Mesophase

Monotropic

Nematic

Optical texture
A mesophase that forms both when heated from the crystalline phase and cooled from the isotropic liquid.

A metallomesogen that incorporates one or more lanthanides.

A type of liquid crystal in which mesophase formation is driven by the effects of a solvent and temperature.

A molecule that exhibits thermotropic mesophases.

Forms mesophases (typically describes molecules or chemical moieties but often interchangeable with "mesomorphic").

Forms mesophases (typically describes materials or phases but often interchangeable with "mesogenic").

An intermediate phase between crystalline and isotropic liquid which is partially ordered and fluid.

A thermotropic mesophase that only forms on cooling from the isotropic liquid.

A mesophase in which there is no translational order, but orientational order is still present.

The pattern formed by a birefringent material when observed using POM. 
Phonon A quantised lattice vibration in a crystal.

Photochromism

A reversible structural change in a material due to excitation by electromagnetic radiation.

Pitch The repeat distance of a helix.

A partially amorphous solid. It is distinct from a liquid crystal in

Plastic crystal that it is not fluid, and can be thought of as a partially ordered glass.

Polycatenar

A mesogen with an elongated core with many tails attached at the ends of the core.

Salicylaldimine

A 2-hydroxybenzaldimine derivative synthesised by the condensation of a salicylaldehyde derivative and an amine.

Scattering vector The spacing of reciprocal lattice points in reciprocal space.

A mesophase which possesses orientational order and

Smectic translational order in one dimension. Can be thought of as a layered liquid.

Tail

A flexible moiety (typically an alkyl chain) extending out from the core of a mesogen.

Tautomerism

Presence of a dynamic equilibrium in a molecule between two isomers (known as tautomers).

Thermotropic

A type of liquid crystal in which mesophase formation is driven by the temperature.

The angle between the average director and the normal vector to the plane in a smectic $C$ mesophase.

Vitrification The conversion of a fluid state into a glassy (amorphous) solid. 


\section{Appendix A \\ Introduction to DSC and POM}

This appendix contains a brief introduction to the theory and use of differential scanning calorimetry (DSC) and polarized optical microscopy (POM).

\section{A.1. Differential Scanning Calorimetry (DSC)}

DSC is a technique that measures the difference in heat flow into a sample and an inert reference as a function of raising or lowering the temperature. It is used to monitor changes in the sample which occur due to changes in temperature, such as phase changes. A simplified schematic of how DSC works is shown in Figure A.1.1.

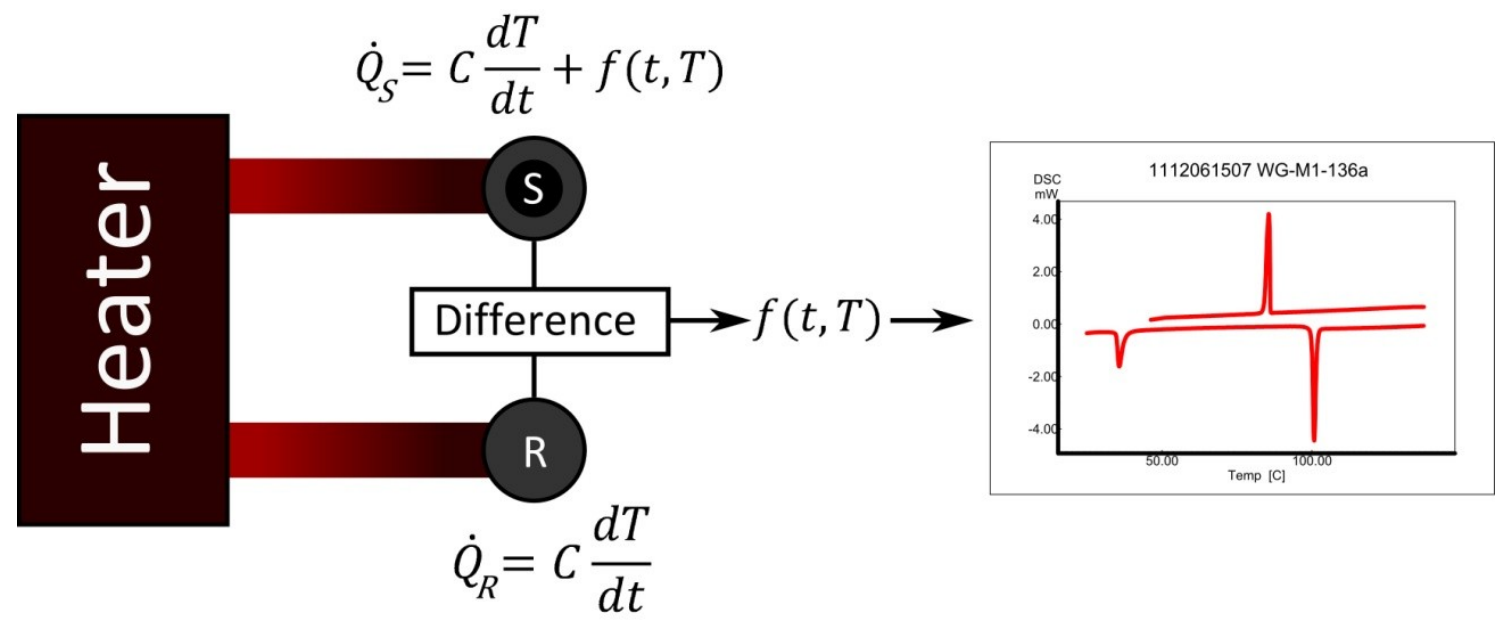

Figure A.1.1: A simplified schematic of DSC in which heat is provided by the heater to the sample (S) and reference $(R)$ and the difference in the heat flow required to change the temperature is measured.

The heat flow $\left(\dot{Q}_{S}\right)$ into the sample has two components:

$$
\dot{Q}_{s}=C_{s} \frac{d T}{d t}+f(t, T)
$$

The first component (in red) describes the heat flow required to change the temperature of the sample. It incorporates the heat capacity of the sample $\left(C_{s}\right)$ and the rate of change of temperature $(d T / d t)$. In an ideal experiment, this component would be the same in both the inert reference and the sample as their heat capacities would be approximately the same. Consequently, when the differential heat flow is measured, this component is removed (Figure A.1.1), leaving only the second 
component (in blue), which results from heat flow due to the heat of a transition in the sample.

A peak in the differential heat flow results from the latent heat of a phase transition. As such, measurement of the differential heat flow allows precise measurement of the temperatures of phase transitions. In addition, DSC is quantitative with respect to measurements of the heat of a transition by measuring the integral of the peaks. The numerical value of the heat is found by calibrating the instrument with a sample with a known enthalpy of fusion (typically indium metal).

DSC is used extensively in liquid crystal research to find the temperatures and enthalpies of phase transitions with extreme precision. ${ }^{4,20,26,54}$ There are, however, some issues in its application to liquid crystals. Unlike some other techniques, DSC is unable to confirm that a mesophase has formed; it can only show that multiple phase transitions have occurred. The advantage it has over other techniques, though, is that sample masses are much lower so transition temperatures can be determined with much more accuracy. However, the low sample mass can make some mesophase transitions difficult to detect due to their low energy. This is particularly true when a transition results from very subtle changes in the order of a system, such as nematic to isotropic or smectic $C$ to smectic $A$ (Figure A.1.2)

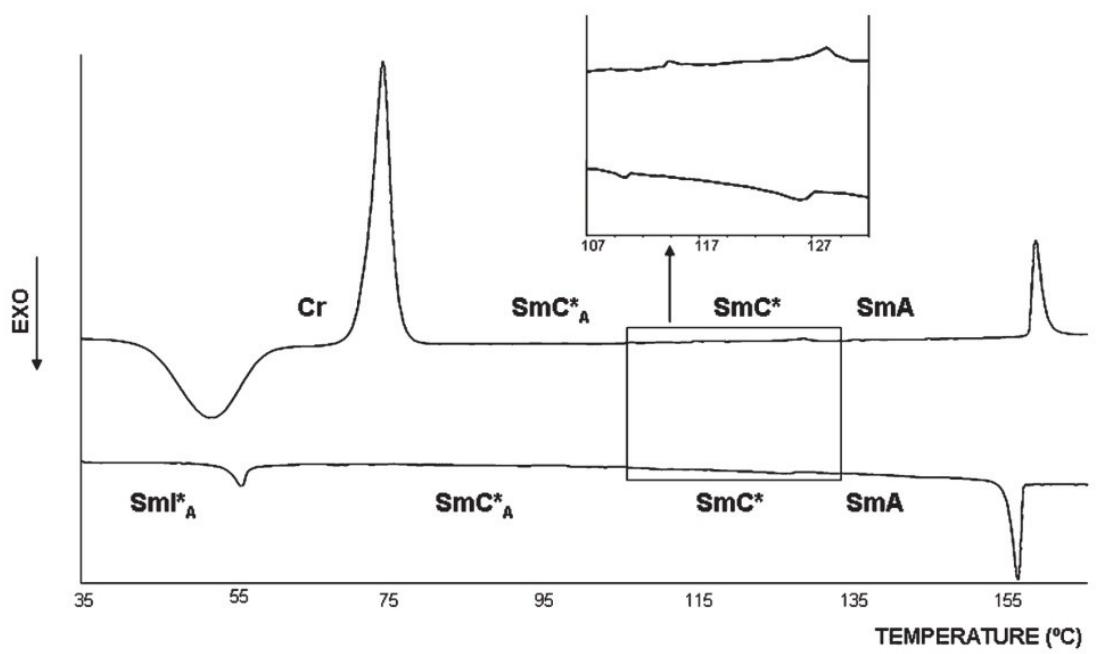

Figure A.1.2: An example of DSC data obtained from a metallomesogen that exhibits multiple smectic mesophases. Reproduced from López de Murillas et al. - "Structure-activity studies of ferroelectric and antiferroelectric imine ligands and their palladium(II) complexes. An antiferroelectric metallomesogen."19 


\section{A.2. Polarised Optical Microscopy (POM)}

Polarised optical microscopy (POM) relies on the birefringence of a mesophase to produce optical textures that can be used to identify that mesophase. It is useful to liquid crystal researchers as it provides an easy means by which a substance can be checked for mesomorphism.

Birefringence is observed when a plane-polarised beam of light is refracted by an anisotropic material into two orthogonally polarised beams. This is due to the material having two refractive indices, one parallel and one perpendicular to an "optical axis" ${ }^{67}$ In an oriented mesophase, the director field of the mesophase acts as the optical axis $^{4,20}$ meaning that liquid crystals can effectively rotate plane polarised light when it is not polarised either perpendicular or parallel to the director field. (Figure A.2.1 (c)). This property is essential for their application in LCDs. ${ }^{36}$
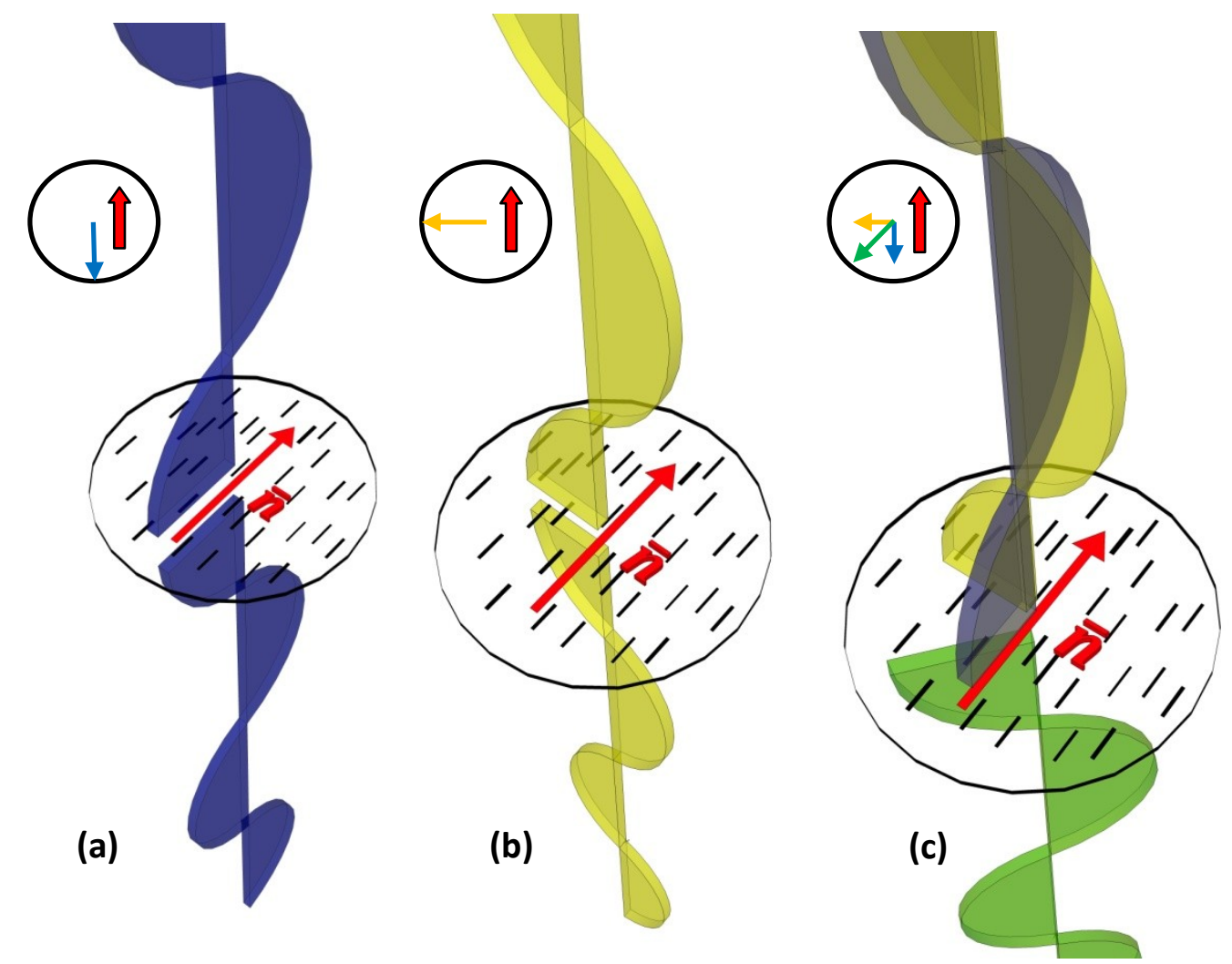

Figure A.2.1: Schematics of (a) refraction of light polarised parallel to the director field, (b) refraction of light polarised perpendicular to the director field and (c) birefringence when the incident beam (green) is split into its components parallel (blue) and perpendicular (yellow) to the average director.

In a polarising microscope, the sample is placed between two crossed polarising filters. The first is called the polariser and the second the analyser ${ }^{83}$ (Figure A.2.2). If the director field in the sample is aligned parallel to either filter, then no birefringence is observed, consequently the sample appears dark. ${ }^{83}$ However, if the director field is not 
aligned parallel with either filter, the sample will be birefringent. The incident polarised beam is split into two beams polarised parallel and perpendicular to the director field (Figure A.2.1(c)). Components of these beams are not removed by the analyser, consequently the sample appears brighter. ${ }^{83}$ This effect is maximised when the director field is at an angle of $45^{\circ}$ to the polariser and analyser as at this angle the component of the refracted beams removed by the analyser is minimised ${ }^{83}$ (Figure A.2.2). In addition to the effect on the brightness of the image, the recombination of the beams in the analyser can lead to interference between the two beams. This only occurs at certain wavelengths and is orientation dependant, meaning that there is variation in colour, as well as brightness, as the sample is rotated. ${ }^{83}$

\section{Analyser}
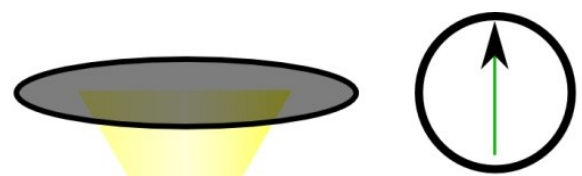

\section{Sample}
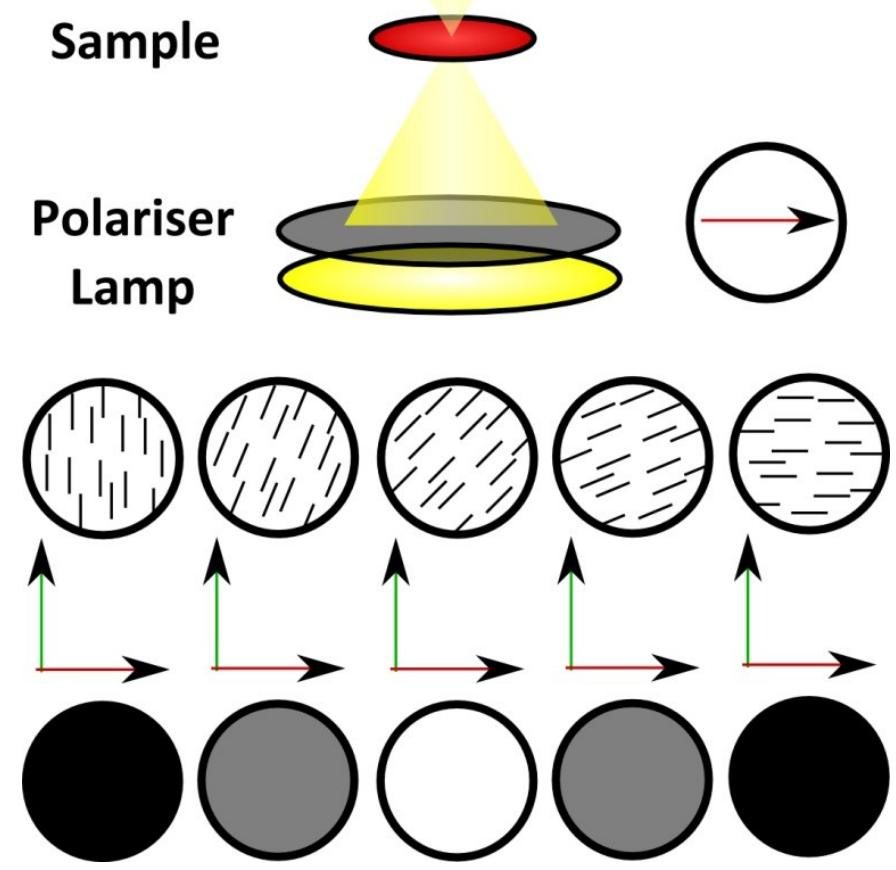

Figure A.2.2: (top) a schematic of a POM system with the orientation of the polariser and analyser shown in profile view, and (bottom) an illustration of the effect of director field orientation relative to the polariser and analyser (red and green respectively) on what is observed through the microscope.

In most liquid crystal samples the mesophase is not aligned over the whole sample, but rather in small domains within the sample. The behaviour of the director field at the boundaries of these domains and around interfaces and contaminants gives rise to line defects in the director field called disclinations. The interactions of disclinations can result in complex optical textures when examined using POM, which can be used to identify a mesophase. ${ }^{4}$ If the microscope is fitted with a hot stage to heat the sample, 
one can use POM as a complimentary technique to DSC to monitor the phase behaviour. There are many different optical textures that liquid crystals exhibit, the two discussed in this study are the Schlieren texture arising from a nematic mesophase (Figure 5.5.3) and the focal conic texture arising from a smectic A mesophase (Figure 5.5.10). The reader is referred to Liquid Crystals by Chandrasekhar ${ }^{4}$ for a comprehensive discussion of the origins of these textures. 


\section{References}

1 Monahan, N., Victoria University of Wellington, 2010 (acknowledgements section).

2 Kelker, H. \& Scheurle, B. A Liquid-crystalline (Nematic) Phase with a Particularly Low Solidification Point. Angew. Chem. Int. Ed. 8, 884-885 (1969).

3 Giménez, R., Lydon, D.P., \& Serrano, J.L. Metallomesogens: a promise or a fact? Curr. Opin. Solid State Mater. Sci. 6, 527-535 (2002).

4 Chandrasekhar, S., Liquid Crystals, 2 ed. (Cambridge University Press, Cambridge, 1992).

5 Date, R.W., Iglesias, E.F., Rowe, K.E., Elliott, J.M., \& Bruce, D.W. Metallomesogens by ligand design. Dalton Trans., 1914-1931 (2003).

6 Reinitzer, F. Beiträge zur Kenntniss des Cholesterins. Monatsh. Chem. 9, 421-441 (1888).

7 Tsai, C., Lin, L.H., \& Kwan, C.C. Surface properties and morphologies of pheohydrane/liquid crystal moisturizer product. Int. J. Cosmetic Sci. 32, 258-265 (2010).

8 Sluckin, T.J., Dunmur, D.A., \& Stegemeyer, H., Crystals that flow: classic papers from the history of liquid crystals. (Taylor \& Francis, 2004).

9 Remde, K. \& Sanders, A., Seismic Change LCD Market Instigated by iPhone, Game Consoles, Auto Displays, According to New Research; accessed on 15 May 2012; available at http://www.sys-con.com/node/2250890, (2012).

10 Donnio, B. Lyotropic metallomesogens. Curr. Opin. Colloid Interface Sci. 7, 371-394 (2002).

11 Hoshino, N. Liquid crystal properties of metal-salicylaldimine complexes. Chemical modifications towards lower symmetry. Coord. Chem. Rev. 174, 77-108 (1998).

12 Yager, K., Introduction to Liquid Crystals; accessed on 9 May 2011; available at http://barrett-group.mcgill.ca/teaching/liquid crystal/LC01.htm, (2006).

13 De Gennes, P.G. \& Prost, J., The physics of liquid crystals. (Clarendon Press, 1995).

14 Coles, H.J. \& Pivnenko, M.N. Liquid crystal 'blue phases' with a wide temperature range. Nature 436, 997-1000 (2005).

15 Etchegoin, P. Blue phases of cholesteric liquid crystals as thermotropic photonic crystals. Phys. Rev. E 62, 1435 (2000).

16 Samsung Develops World's First 'Blue Phase' Technology to Achieve $240 \mathrm{~Hz}$ Driving Speed for High-Speed Video; accessed on 10 May 2011; available at http://www.physorg.com/news129997960.html, (2008).

17 Laschat, S., Baro, A., Steinke, N., Giesselmann, F., Hägele, C., Scalia, G., Judele, R., Kapatsina, E., Sauer, S., Schreivogel, A., \& Tosoni, M. Discotic Liquid Crystals: From Tailor-Made Synthesis to Plastic Electronics. Angew. Chem. Int. Ed. 46, 4832-4887 (2007). 
18 Jiles, D., Introduction to the electronic properties of materials. (Nelson Thornes, 2001).

19 Lopez de Murillas, D., Pinol, R., Blanca Ros, M., Serrano, J.L., Sierra, T., \& Rosario de la Fuente, M. Structure-activity studies of ferroelectric and antiferroelectric imine ligands and their palladium(II) complexes. An antiferroelectric metallomesogen. J. Mater. Chem. 14, 1117-1127 (2004).

20 Serrano, J.L., Metallomesogens: Synthesis, Properties, and Applications. (VCH, Weinheim, 1996).

21 Circu, V. \& Dumitrascu, F. Dinuclear ortho-Metallated Palladium(II) Complexes with Acetato and Chloro Bridges as Liquid Crystals. Mol. Cryst. Liq. Cryst. 534, 41-49 (2011).

22 Liao, C.T., Chen, H.H., Hsu, H.F., Poloek, A., Yeh, H.H., Chi, Y., Wang, K.W., Lai, C.H., Lee, G.H., Shih, C.W., \& Chou, P.T. Mesomorphism and Luminescence Properties of Platinum(II) Complexes with Tris(alkoxy)phenyl-Functionalized Pyridyl Pyrazolate Chelates. Chem. Eur. J. 17, 546-556 (2011).

23 Mocanu, A.S., Ilis, M., Dumitrascu, F., Ilie, M., \& Circu, V. Synthesis, mesomorphism and luminescence properties of palladium(II) and platinum(II) complexes with dimeric Schiff base liquid crystals. Inorg. Chim. Acta 363, 729-736 (2010).

24 Wang, Y., Liu, Y., Luo, J., Qi, H., Li, X., Nin, M., Liu, M., Shi, D., Zhu, W., \& Cao, Y. Metallomesogens based on platinum(ii) complexes: synthesis, luminescence and polarized emission. Dalton Trans. 40, 5046-5051 (2011).

25 Binnemans, K. Luminescence of metallomesogens in the liquid crystal state. J. Mater. Chem. 19, 448-453 (2009).

26 Yelamaggad, C.V., Prabhu, R., Shanker, G., \& Bruce, D.W. Optically active, mesogenic lanthanide complexes: design, synthesis and characterization. Liq. Cryst. 36, 247-255 (2009).

27 Cardinaels, T., Driesen, K., Parac-Vogt, T.N., Heinrich, B.t., Bourgogne, C., Guillon, D., Donnio, B., \& Binnemans, K. Design of High Coordination Number Metallomesogens by Decoupling of the Complex-Forming and Mesogenic Groups: Nematic and Lamello-Columnar Mesophases. Chem. Mater. 17, 6589-6598 (2005).

28 Saez, I.M., Goodby, J.W., \& Richardson, R.M. A Liquid-Crystalline Silsesquioxane Dendrimer Exhibiting Chiral Nematic and Columnar Mesophases. Chem. Eur. J. 7, 2758-2764 (2001).

29 Dardel, B., Guillon, D., Heinrich, B., \& Deschenaux, R. Fullerene-containing liquidcrystalline dendrimers. J. Mater. Chem. 11, 2814-2831 (2001).

30 Bruce, D.W. Calamitics, cubics, and columnars. Liquid-crystalline complexes of silver(I). Acc. Chem. Res. 33, 831-840 (2000).

31 Hird, M. \& Toyne, K.J. Fluoro Substitution in Thermotropic Liquid Crystals. Mol. Cryst. Liq. Cryst. 323, 1 - 67 (1998).

32 Galyametdinov, Y.G., Malykhina, L.V., Haase, W., Driesen, K., \& Binnemans, K. Preliminary Communication: Luminescent Lanthanide Complexes with Liquid Crystalline Properties. Liq. Cryst. 29, 1581-1584 (2002). 
33 Giroud-Godquin, A.M. My 20 years of research in the chemistry of metal containing liquid crystals. Coord. Chem. Rev. 178-180, 1485-1499 (1998).

34 Fazio, D., Mongin, C., Donnio, B., Galerne, Y., Guillon, D., \& Bruce, D.W. Bending and shaping: cubics, calamitics and columnars. J. Mater. Chem. 11, 2852-2863 (2001).

35 Guillon, D., Heinrich, B., Ribeiro, A.C., Cruz, C., \& Nguyen, H.T. Thermotropic Lamellar-to-Columnar Phase Transition Exhibited by a Biforked Compound. Mol. Cryst. Liq. Cryst. 317, 51-64 (1998).

36 Kawamoto, H. The history of liquid-crystal displays. P. IEEE 90, 460-500 (2002).

37 Lakowicz, J.R., Principles of fluorescence spectroscopy. (Springer, 2006).

38 Bhattacharjee, C.R., Das, G., Mondal, P., \& Rao, N.V.S. Novel photoluminescent hemi-disclike liquid crystalline $\mathrm{Zn}$ (II) complexes of [N2O2] donor 4-alkoxy substituted salicyldimine Schiff base with aromatic spacer. Polyhedron 29, 30893096 (2010).

39 Bhattacharjee, C.R., Das, G., Goswami, P., Mondal, P., Prasad, S.K., \& Rao, D.S.S. Novel photoluminescent lanthanidomesogens forming bilayer smectic phase derived from blue light emitting liquid crystalline, one ring O-donor Schiff-base ligands. Polyhedron 30, 1040-1047 (2011).

40 Vieira, A.A., Gallardo, H., Barbera, J., Romero, P., Serrano, J.L., \& Sierra, T. Luminescent columnar liquid crystals generated by self-assembly of $1,3,4-$ oxadiazole derivatives. J. Mater. Chem. 21, 5916-5922 (2011).

41 Szerb, E.I., Talarico, A.M., Aiello, I., Crispini, A., Godbert, N., Pucci, D., Pugliese, T., \& Ghedini, M. Red to Green Switch Driven by Order in an Ionic Ir-III LiquidCrystalline Complex. Eur. J. Inorg. Chem. 2010, 3270-3277 (2010).

42 Shin, C.H., Huh, J.O., Lee, M.H., \& Do, Y. Polymorphism-induced dual phosphorescent emission from solid-state iridium(III) complex. Dalton Trans., 6476-6479 (2009).

43 Sakurai, T., Tashiro, K., Honsho, Y., Saeki, A., Seki, S., Osuka, A., Muranaka, A., Uchiyama, M., Kim, J., Ha, S., Kato, K., Takata, M., \& Aida, T. Electron- or HoleTransporting Nature Selected by Side-Chain-Directed $\pi$-Stacking Geometry: Liquid Crystalline Fused Metalloporphyrin Dimers. J. Am. Chem. Soc. 113, 6537-6540 (2011).

44 Kittel, C., Introduction to solid state physics, 7 ed. (Wiley, New York, 1996).

45 Lemaur, V., da Silva Filho, D.A., Coropceanu, V., Lehmann, M., Geerts, Y., Piris, J., Debije, M.G., van de Craats, A.M., Senthilkumar, K., Siebbeles, L.D.A., Warman, J.M., Brédas, J.L., \& Cornil, J. Charge Transport Properties in Discotic Liquid Crystals: A Quantum-Chemical Insight into Structure-Property Relationships. J. Am. Chem. Soc. 126, 3271-3279 (2004).

46 Suárez, S., Imbert, D., Gumy, F., Piguet, C., \& Bünzli, J.C.G. Metal-Centered Photoluminescence as a Tool for Detecting Phase Transitions in Eu"I- and Tb"IContaining Metallomesogens. Chem. Mater. 16, 3257-3266 (2004).

47 Terazzi, E., Torelli, S., Bernardinelli, G., Rivera, J.P., Bénech, J.M., Bourgogne, C., Donnio, B., Guillon, D., Imbert, D., Bünzli, J.C.G., Pinto, A., Jeannerat, D., \& Piguet, 
C. Molecular Control of Macroscopic Cubic, Columnar, and Lamellar Organizations in Luminescent Lanthanide-Containing Thermotropic Liquid Crystals. J. Am. Chem. Soc. 127, 888-903 (2004).

48 Driesen, K., Vaes, C., Cardinaels, T., Goossens, K., Gorller-Walrand, C., \& Binnemans, K. Polarized Luminescence of Non-mesogenic Europium(III) Complexes Doped into a Nematic Liquid Crystal. J. Phys. Chem. B 113, 10575-10579 (2009).

49 Nobelprize.org, The Nobel Prize in Chemistry 2010 - Scientific Background; accessed on 25 May 2011; available at http://nobelprize.org/nobel prizes/chemistry/laureates/2010/sci.html, (2010).

50 Paschke, R., Liebsch, S., Tschierske, C., Oakley, M.A., \& Sinn, E. Synthesis and Mesogenic Properties of Binuclear Copper(II) Complexes Derived from Salicylaldimine Schiff Bases. Inorg. Chem. 42, 8230-8240 (2003).

51 Blake, A.B., Chipperfield, J.R., Hussain, W., Paschke, R., \& Sinn, E. Effects of Ligand Substituents ( $F$ for $H$; OR for $R$ ) on Mesogenic Properties of $M($ Salen) Derivatives $(\mathrm{M}=\mathrm{Cu}, \mathrm{Ni}, \mathrm{VO})$. New Fluoro-Substituted Complexes and Crystal Structure of the Mesogen Ni(5-hexylSalen). Inorg. Chem. 34, 1125-1129 (1995).

52 Bhattacharjee, C.R., Das, G., \& Mondal, P. Novel non-discoid chiral copper(II)-salen type $\left[\mathrm{N}_{2} \mathrm{O}_{2}\right]$ donor Schiff base complexes with a cyclohexane diamine spacer: synthesis, electrochemistry, columnar mesomorphism and DFT study. Liq. Cryst. 38, 441 - 449 (2011).

53 Bhattacharjee, C.R., Datta, C., Das, G., Chakrabarty, R., \& Mondal, P. Induction of photoluminescence and columnar mesomorphism in hemi-disc salphen type Schiff bases via nickel(II) coordination. Polyhedron 33, 417-424 (2012).

54 Bhattacharjee, C.R., Das, G., \& Mondal, P. Liquid-crystalline oxovanadium(IV) complexes accessed from bidentate [N, O] donor salicylaldimine Schiff-base ligands. J. Coord. Chem. 64, 3273-3289 (2012).

55 Ghedini, M., Armentano, S., Bartolino, R., Kirov, N., Petrov, M., \& Nenova, S. Texture and Calorimetric Investigations of Some New Copper(II) CoordinationCompounds with Mesomorphic Properties. J. Mol. Liq. 38, 207-213 (1988).

56 Ghedini, M., Morrone, S., Gatteschi, D., \& Zanchini, C. Transition-Metals Complexed to Ordered Mesophases. Synthesis, Mesomorphic Behavior, and EPR Investigations on a Homologous Series of $\mathrm{N}$-(4-Alkoxysalicylidene)-4'-Alkylanilines Complexed to Copper(II). Chem. Mater. 3, 752-758 (1991).

57 Van Deun, R. \& Binnemans, K. Mesomorphic lanthanide complexes with azomethine ligands. J. Alloys Compd. 303-304, 146-150 (2000).

58 Silverstein, R.M., Webster, F.X., \& Kiemle, D.J., Spectrometric identification of organic compounds, 7 ed. (John Wiley \& Sons, New York, 2005).

59 Bhattacharjee, C.R., Das, G., \& Mondal, P. Photoluminescent Hemidisc-Shaped Liquid Crystalline Nickel(II) Schiff-Base Complexes. Eur. J. Inorg. Chem. 2011, 53905396 (2011).

60 Percy, G.C. \& Thornton, D.A. N-alkyl salicylaldimine complexes: Infrared and PMR spectra of the ligands and vibrational frequencies of their metal(II) chelates. J. Inorg. Nucl. Chem. 34, 3369-3376 (1972). 
61 Kovacic, J.E. The $\mathrm{C}=\mathrm{N}$ stretching frequency in the infrared spectra of Schiff's base complexes--I. Copper complexes of salicylidene anilines. Spectrochim. Acta, Part A 23, 183-187 (1967).

62 Schnablegger, H. \& Singh, Y., A Practical Guide to SAXS - Getting Acquainted With the Principles. (Anton Paar, Graz, 2006).

63 Sheldrick, G. A short history of SHELX. Acta Crystallogr., Sect. A: Found. Crystallogr. 64, 112-122 (2008).

64 Etchegoin, P., Fainstein, A., \& Pregliasco, R.G. Optical nonlinearities in the supercooled phase of nematic liquid crystal drops. Physica D 134, 144-151 (1999).

65 Ghedini, M., Armentano, S., Bartolino, R., Rustichelli, F., Torquati, G., Kirov, N., \& Petrov, M. New Liquid-Crystalline Compounds Containing Transition-Metals. Mol. Cryst. Liq. Cryst. 151, 75-91 (1987).

66 Meyer, C., Le Cunff, L., Belloul, M., \& Foyart, G. Focal Conic Stacking in Smectic A Liquid Crystals: Smectic Flower and Apollonius Tiling. Materials 2, 499-513 (2009).

67 Ashcroft, N.W. \& Mermin, N.D., Solid State Physics. (Harcourt College Publishers, Orlando, 1976).

68 Spotts, M.F., Design of machine elements, 4 ed. (Pearson Prentice Hall, Englewood Cliffs, 1971).

69 Wakelin, J.H., Sutherland, A., \& Beck, L.R. Linear thermal expansion coefficients for the crystalline phase in high polymers. J. Polym. Sci. 42, 278-280 (1960).

70 Samsung mulls LCD unit spin-off amid falling demand; accessed on 15 May 2012; available at http://www.bbc.co.uk/news/business-17037742, (2012).

71 Frisch, M.J., Trucks, G.W., Schlegel, H.B., Scuseria, G.E., Robb, M.A., Cheeseman, J.R., Scalmani, G., Barone, V., Mennucci, B., Petersson, G.A., Nakatsuji, H., Caricato, M., Li, X., Hratchian, H.P., Izmaylov, A.F., Bloino, J., Zheng, G., Sonnenberg, J.L., Hada, M., Ehara, M., Toyota, K., Fukuda, R., Hasegawa, J., Ishida, M., Nakajima, T., Honda, Y., Kitao, O., Nakai, H., Vreven, T., Montgomery, J., J. A., Peralta, J.E., Ogliaro, F., Bearpark, M., Heyd, J.J., Brothers, E., Kudin, K.N., Staroverov, V.N., Kobayashi, R., Normand, J., Raghavachari, K., Rendell, A., Burant, J.C., lyengar, S.S., Tomasi, J., Cossi, M., Rega, N., Millam, J.M., Klene, M., Knox, J.E., Cross, J.B., Bakken, V., Adamo, C., Jaramillo, J., Gomperts, R., Stratmann, R.E., Yazyev, O., Austin, A.J., Cammi, R., Pomelli, C., Ochterski, J.W., Martin, R.L., Morokuma, K., Zakrzewski, V.G., Voth, G.A., Salvador, P., Dannenberg, J.J., Dapprich, S., Daniels, A.D., Farkas, Ö., Foresman, J.B., Ortiz, J.V., Cioslowski, J., \& Fox, D.J., Gaussian 09, Revision B.01 (Gaussian, Inc, Wallingford, 2009).

72 Jones, M., Organic Chemistry, 3 ed. (W. W. Norton \& Company Ltd, New York, 2005).

73 Weast, R.C. \& Selby, S.M., CRC Handbook of Chemistry and Physics: A Readyreference Book of Chemical and Physical Data, 48 ed. (CRC Press, Cleveland, 1968).

74 Hammud, H.H., Ghannoum, A., \& Masoud, M.S. Spectral regression and correlation coefficients of some benzaldimines and salicylaldimines in different solvents. Spectrochim. Acta, Part A 63, 255-265 (2006). 
75 Vafazadeh, R., Hayeri, V., \& Willis, A.C. Synthesis, crystal structure and electronic properties of bis(N-2-bromophenyl-salicydenaminato)copper(II) complex. Polyhedron 29, 1810-1814 (2010).

76 Zhao, J., Zhao, B., Liu, J., Xu, W., \& Wang, Z. Spectroscopy study on the photochromism of Schiff Bases N,N'-bis(salicylidene)-1,2-diaminoethane and N,N'bis(salicylidene)-1,6-hexanediamine. Spectrochim. Acta, Part A 57, 149-154 (2001).

77 Aylward, G.H. \& Findlay, T.J.V., Si Chemical Data, 5 ed. (Wiley, Milton, 2002).

78 Tas, E., Kilic, A., Konak, N., \& Yilmaz, I. The sterically hindered salicylaldimine ligands with their copper(II) metal complexes: Synthesis, spectroscopy, electrochemical and thin-layer spectroelectrochemical features. Polyhedron 27, 1024-1032 (2008).

79 Tamaoki, N. \& Kamei, T. Reversible photo-regulation of the properties of liquid crystals doped with photochromic compounds. J. Photochem. Photobiol., C 11, 4761 (2010).

80 Wu, M.-H., Chu, C.-C., Cheng, M.-C., \& Hsiao, V.K.S. Reversible Phase Transition and Rapid Switching of Azobenzene-Doped Cholesteric Liquid Crystals with a Single Laser. Mol. Cryst. Liq. Cryst. 557, 176-189 (2012).

81 Ulusoy, M., Şahin, O., Kilic, A., \& Büyükgüngör, O. Multinuclear Cu(II) Schiff Base Complex as Efficient Catalyst for the Chemical Coupling of $\mathrm{CO}_{2}$; and Epoxides: Synthesis, X-ray Structural Characterization and Catalytic Activity. Catal. Lett. 141, 717-725 (2011).

82 Bhattacharjee, C.R., Datta, C., Das, G., \& Mondal, P. Liquid crystalline dinuclear copper(II) complexes accessed from photoluminescent tridentate [ONO]-donor Schiff base ligands. Liq. Cryst., 1-8 (2012).

83 Murphy, D.B., Fundamentals of Light Microscopy and Electronic Imaging. (WileyLiss, New York, 2001). 